\title{
Landlord Project Multi-Year Work Plan Fiscal Year 1998 WBS 1.5
}

DynCorp Tri-Cities Services, Inc.

September 1997

Prepared for the U.S. Department of Energy

Assistant Secretary for Environmental. Management 
RELEASE AUTHORIZATION

Document Number: HNF-SP-1242

Document Title: Landlord Project Multi-Year Work Plan

- Fiscai Year 1998

This document, reviewed in accordance with DOE Order 1430.1D, "Scientific and Technical Information Management," and DOE G 1430.1D-1, "Guide to the Management of Scientific and Technical Information," does not contain classified or sensitive unclassified information and is:

APPROVED FOR PUBLIC RELEASE

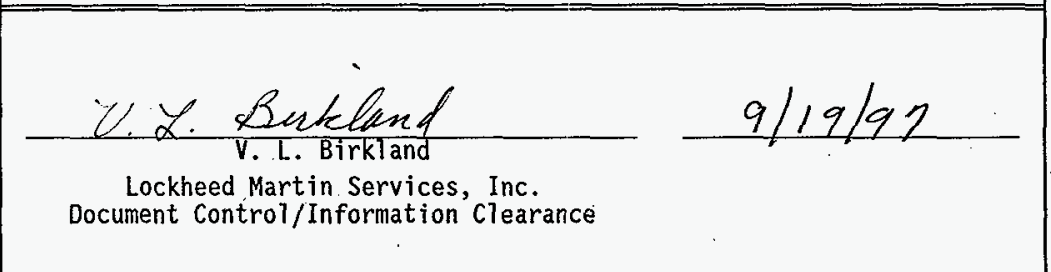

Reviewed for Applied tachnology, Business Sensitive, Classified, Copynghted, Export Controlled, Patent, Personallphvate, Propriatary, Protected CRADA, Trademark, Unclassified Controlled Nuclear information.

LEGAL DISCLAIMER. This report was prepared as an account of work sponsored by an agency of the United States Government. Neither the United States Government nor any agency thereof, not any of their employeas, nor any of their contractorc, subcontractors or their amployees, makes any warranty, express or implied, or assumes any legat liability or responsibility for the accuracy, completenese, or any third party's use or the results of such use of any infonation, apparatus, product, or process disclosed, or represents that its use would not infringe privately owned rights. Reforence herein to any specific commercial product, process, or service by trado name, trademark, manufacturer, or otherwise, does not necessarily constitute or imply its endoreement, recommendation, or favoring by the United States Govemment or any agency theroof or its contractore or aubcontraotore. The views and opinione of authors expressed herein do not necess arily state or roflest thoso of the United States Govornment or any agency thereof. This report has been reproduced from the best available copy. Printed in the United States of America. Available to the U.S. Department of Energy and its contractors from the U.S. Department of Energy Office of Scientific and Technical information, P.O. Box 62, Oak Ridge, TN 37831; Telephone: 423/576-8401.

Available to the publie from the U.S. Department of Commerce National Technical Information Service, 5285 Port Royal Road, Springfield, VA 22161; - Tolephono: 703/487-4650. 
LANDLORD PROJECT

WAS 1.5

FY 1998 Work Plan

HNF-SP-1242, Rev. 0

MYWP Approval Sheet

Assistant Manager; Facility Transition

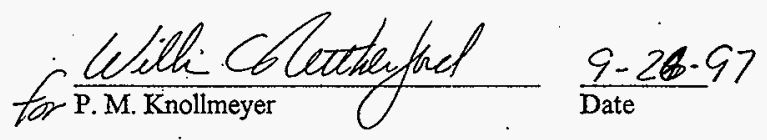

- Director, Site Infrastructure Division

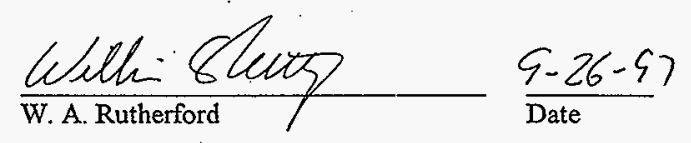

Acting Director, Infrastructure \& Security

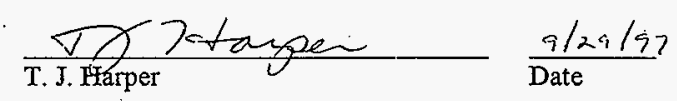

General Manager, DynCorp Tri-Cities Services, Inc.

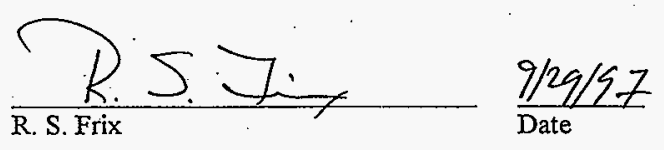




\section{LANDLORD ]PROJECT \\ WBS 11.5}

FY 1998 Work Plan

HNF-SP-1242, Rev. 0

\section{EXECUTTVE SUMMARY}

The Fiscal Year 1998 Landlord Project Multi-Year Work Plan (MYWP) addresses the mission objectives, workscope, work breakdown structures (WBS), management approach, and resource requirements for general infrastructure support.

The primary assumptions within this MYWP are:

- No demolition workscope is funded in either FY 1998 or 1999 upon the direction of the Site Management Board and the Regulators

- Major system replacements are not anticipated nor funded at the current level

- All remaining ongoing line item projects will complete during FY 1998

- One new line item start in FY 2001 for the Solid Sanitary Landfill Cap is shown as multi-year funded due to the current funding projections

- Emphasis on completing actual work rather than paper planning activities

- Additional unanticipated funding will be invested in compliance related mortgage reduction activities

- No funding will be provided beyond FY 1998 for Lockheed Martin Services, Inc. (LMSI) equipment replacements per DOE-RL direction.

- Shutdown of the $284 \mathrm{E}$ and 384 Powerhouses is currently planned on a 120 working day duration which is the least cost option to the Government.

- The priorities reflected in this MYWP were arrived at utilizing a modified version of the Capital Asset Management Process. This process employs prioritization based on risk to the public, workers, and the environment, as well as overall cost reduction to the site.

- Scope for the shutdown and isolation of the $284 \mathrm{~W}$ package boiler is not included in this MYWP.

The project is based on the following priorities: 1) Core Infrastructure Maintenance; 2) Infrastructure Mortgage Reduction; and 3) remaining line item projects requiring their own ADS. Of these, Core Infrastructure Maintenance provides the funding to respond to the mission essential elements of the infrastructure systems. Infrastructure Mortgage Reduction responds to the need to reduce outyear mortgages in general purpose facilities by vacating, and isolating the utilities in an effort to reduce the outyear costs of surveillance and maintenance. It also encompasses the legacy equipment disposition for compliance with RCRA and other regulations.

\section{Strategies:}

- Identify and understand infrastructure requirements including core capabilities to be retained.

- Determine the best alternative (competitive with the best commercial services) for continuing to provide the required infrastructure.

- Implement the best alternative considering capability, capacity, safety, and reliability.

- Continue to optimize the infrastructure wherever possible to reduce the overall cost to the Hanford Site allowing additional funds to be expended on cleanup activities. 


\section{Landlord Program Strategy}

\section{Fundamental Building Blocks}

\section{- Mortgage Reduction}

- Demolition

- Legacy Equipment Disposition

- Surveillance \& Maintenance of Vacant Facilities

- Utilities Isolation for Vacant Facilities

\section{- Core Infrastructure Maintenance}

- Roads

- Water and Sewer System Replacements/Refurbishment

- Emergency Preparedness

- Electrical System Replacements/Refurbishment

- Telecommunications System Replacements/Refurbishment

- Facility, Lab, and Shop Replacements/Repairs

- Business Equipment Replacements (HLAN service, voice Messaging, etc.)

\section{The Landlord Program 10 Year Plan Supports:}

- Maintaining Minimum Safe Level of Infrastructure Support

- Significant Savings to the Department of Energy by Reducing the Site Mortgage

- Surveillance \& Maintenance Cost Avoidance $=\$ 1$ per square foot of savings per year

- Utilities Costs Avoidance $=$ min. of $\$ 5 \mathrm{~K}$ to $\$ 8 \mathrm{~K}$ per building savings per year (some buildings significantly more)

- Mitigating Stakeholder Concerns (Ecology for Sanitary Sewers and RCRA Violations)

- Large Return on Investment 


\section{LANDLORD PROJECT \\ WBS 1.5}

\section{CONTENTS}

\section{SECTION A - PROJECT SUMMARY SECTION}

\section{A.1.0 Technical Baseline}

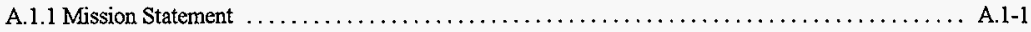

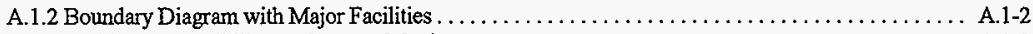

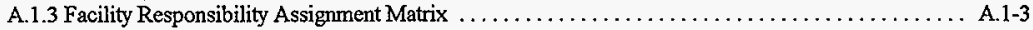

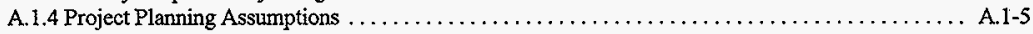

A.2.0 Project Hanford Breakdown Structure Hierarchy

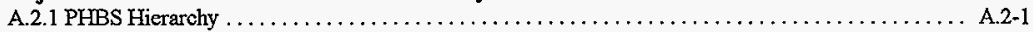

A.3.0 Schedule Baseline

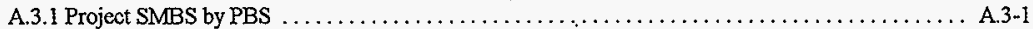

A.4.0 Cost Baseline

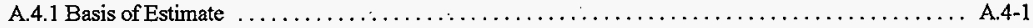

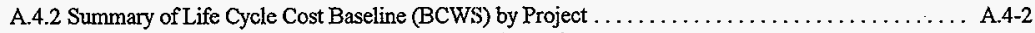

A.4.3 Summary of Life Cycle Budget Authority $(B / A)$ by Project $\ldots \ldots \ldots \ldots \ldots \ldots \ldots \ldots \ldots \ldots \ldots \ldots \ldots \ldots \ldots$

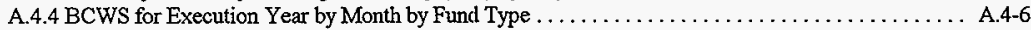

A.4.5 BCWS for Execution Year by Month by Cost Element $\ldots \ldots \ldots \ldots \ldots \ldots \ldots \ldots \ldots \ldots \ldots$ A.4-7

A.5.0 Performance Measures/Metrics Including Cost Metrics

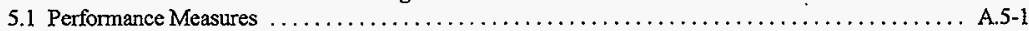

\section{SECTION B - ADDITIONAL SECTIONS AT THE PBS LEVEL}

\section{B.1.0 Landlord}

B.1.1.1 Landlord Organization Mission $\ldots \ldots \ldots \ldots \ldots \ldots \ldots \ldots \ldots \ldots \ldots \ldots \ldots \ldots \ldots \ldots \ldots \ldots, \ldots, 1-1$

\section{B.2.0 WBS}

B.1.1.2 Landlord End Point Targets from Hanford Strategic Plan $\ldots \ldots \ldots \ldots \ldots \ldots \ldots \ldots \ldots \ldots \ldots . . .1-2$

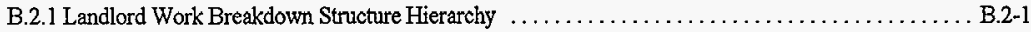

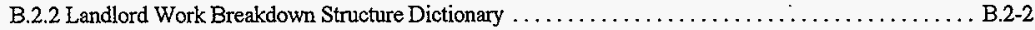

B.2.3 Landlord Responsibility Matrix $\ldots \ldots \ldots \ldots \ldots \ldots \ldots \ldots \ldots \ldots \ldots \ldots \ldots \ldots \ldots \ldots \ldots \ldots \ldots \ldots \ldots \ldots$, B.2-7

\section{B.3.0 Schedule Baseline}

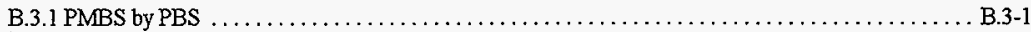

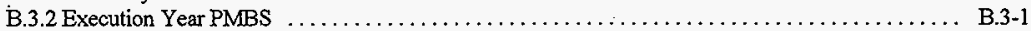

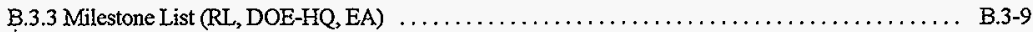

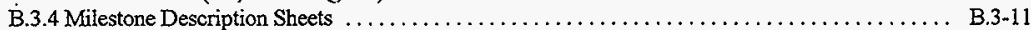

B,4.0 Cost Baseline

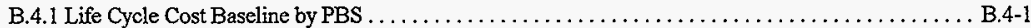

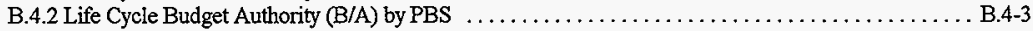

B.4.3 Life Cycle Cost Baseline by Year by Fund Type by PBS $\ldots \ldots \ldots \ldots \ldots \ldots \ldots \ldots \ldots \ldots \ldots, 4.5$

B.4.4 Execution Year Cost Baseline by Month by PBS by ADS $\ldots \ldots \ldots \ldots \ldots \ldots \ldots \ldots \ldots \ldots \ldots . .4 .7$

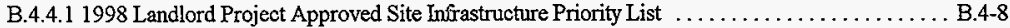

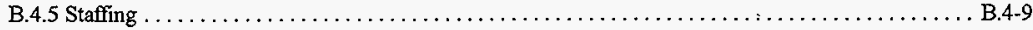

\section{Appendix}

Lower Level WBS Dictionary Sheets

Appendix-1 


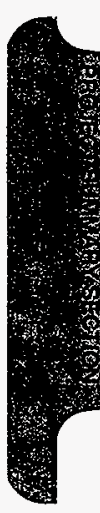


1.0 TECHNICAL BASELINE 


\section{A.1.0 Technical Baseline}

The MYWP technical baseline describes the work to be accomplished by the Project and the technical standards which govern that work.

\section{A.1.1 Mission Statement}

The mission of Landlord Project is to preserve, upgrade, maintain, and forecast cost effective general infrastructure activities to facilitate the Hanford Site cleanup mission. Specific functions and services provided by Landlord Project include utilities (i.e. steam, water, sanitary sewer, solid waste disposal, electrical and telecommunication distribution), transportation, general purpose facilities (includes general support shops and laboratories), services, and energy and land use management. All Landlord Project activities will be performed in an environmentally sound, safe, economical, prudent, and reliable manner. The Hanford Site Landlord Project will be competitive with commercially provided services to offer the best price, quality, and service available.

The major project objectives are:

The overall Landlord Project objective is to maintain a standard of excellence and safety while providing efficient, affordable, and effective infrastructure and support services.

The Project will strive to maintain, preserve, or upgrade strategic assets while consolidating facilities and functions to lower operating and maintenance costs. Realistic goals and strong baseline control are critical to meeting the Project objectives. Paramount to these objectives is compliance with all applicable laws, orders, agreements, codes, standards, and best management and safety practices.

The objectives for general infrastructure support are reflected in two specific areas, 1) Core Infrastructure Maintenance, and 2) Infrastructure Mortgage Reduction.

The following objectives are applicable to the Landlord Project:

Continue to focus on defining and addressing core infrastructure needs. Utilize mortgage reduction (i.e. elimination, excessing, salvage, and demolition) to transition facilities and equipment to their most cost effective status; thereby optimizing the site infrastructure. Provide upgrades to the Site only where cost effective.

Provide an infrastructure that serves the Hanford Mission, leaves diversification value and viable assets, concentrate service locations, excess, disposition, or demolish inadequate and under utilized facilities, promote energy efficiency, and maintain iacilities and equipment as economicall and safely as possible within environmental regulations.

Integrate Occupational Safety and Health Act (OSHA) requirements into the continuous facility inspection project. Ensure compliance with applicable federal and state regulations, and the Tri-Party Agreement.

Identify future infrastructure needs, integrate Landiord Project planning with other project plans, effectively use limited resources, improve project definition and cost estimating, and direct fund all appropriate costs.

Develop a reliable and meaningful process for performing inspections, reporting and life cycle costing of projects for Landlord functions ensuring effective asset management of site resources.

Encourage outreach and partnership with local/private sector and Tribal Governments. 
Provide site wide coordination and management of land use issues to ensure effective support to site cleanup decisions and economic transition initiatives.

\section{A.1.2 Boundary Diagram with Major Facilities}

The following table indentifies the major facilities that interface with this Project. The left column of the table identifies the major facilities that generate waste, materials, or infrastructure for this Project. The right column of the table identifies the major facilities that will receive waste and materials from this Project. The blank center column represents this Project.

Table A.1-1 Landlord Project Boundary Diagram

\begin{tabular}{|l|l|l|}
\hline $\begin{array}{c}\text { Extemal Interfaces } \\
\text { Hanford Legacy } \\
\text { Hanford Site Environmental System Interfaces } \\
\text { None- }\end{array}$ & $\begin{array}{l}\text { Extemal Interfaces } \\
\text { Oftsite Landfill } \\
\text { Offsite Sales } \\
\text { Hanford Site Environmental System Interfaces } \\
\text { None- }\end{array}$ \\
\hline
\end{tabular}


HNF-SP-1242

Rev: 0

Landiord Project

WBS 1.5

27 August 1997

\section{A.1.3 Facility Responsibility Assignment Matrix}

This section provides a table that identifies the sub-projects, major facilities, and the life cycle assignments.

Table A.1-2 Facility Responsibility Assignment Matrix

\begin{tabular}{|c|c|c|c|c|c|c|c|c|c|}
\hline \multirow{3}{*}{ Asset } & \multicolumn{9}{|c|}{ Life cycle } \\
\hline & \multirow{2}{*}{$\begin{array}{l}\text { Program } \\
\text { Planning }\end{array}$} & \multirow{2}{*}{\begin{tabular}{c|} 
Pre \\
Conceptual
\end{tabular}} & \multirow[b]{2}{*}{ Conceptual } & \multicolumn{3}{|c|}{ Execute } & \multirow[b]{2}{*}{$0 \& M$} & \multicolumn{2}{|c|}{ Close Out } \\
\hline & & & & Design & Construction & Tumover & & Post Ops & D\&D \\
\hline Reactors on the River Electrical System & RL-TP13 & & & & & & RL-1114 & RL-TP13 & RL-TP13 \\
\hline Reaclors on the River Water System & RL-TP13 & & & & & & RL-J112 & RL-TP13 & RL-IP13 \\
\hline \begin{tabular}{|l|l}
$\begin{array}{l}\text { Reactors on the River Liquid Sanitary Waste } \\
\text { System }\end{array}$ & F \\
\end{tabular} & RL-TP13 & & & & & & RL-1133 & RL-TP13 & RL-TP13 \\
\hline $\begin{array}{l}\text { Reactors on the River Telecommunicalion } \\
\text { System }\end{array}$ & RL-TP13 & & & & & & RL-I6 & RL-TP13 & RL-TPt3 \\
\hline Reactors on the River Office Facilities & RL-TP13 & & & & & & RL-113 & RL-TP13 & RL-TP13 \\
\hline Reactors on the River Area Storage Facilities $P$ & RL-TP13 & & & & & & RL-17 & RL-TP13 & RL-TP13 \\
\hline Reactors on the River Area Land & RL-TP13 & & & & & & $R L-1123$ & RL-TP13 & RL-TP13 \\
\hline Reactors on the River Road System. & RL-TP13 & & & & & & RiL-1121 & RL-TP13 & RL-TP13 \\
\hline Reactors on the River Rail System & RL-TP13 & & & & & & RL-1122 & RL-TP13 & RL-TP13. \\
\hline 200 Area Treated Effluent Disposal Facility & RL-WM05 & & & & & & RL-WM05 & RL-TP13 & $($ tbd) \\
\hline Centrai Plateau Steam System & RL-TP13 & & & & & & RL-111! & AL-TP13 & RL-TP13 \\
\hline Central Plateau Water System. & RL-JP13 & & & & & & RL-1112 & RL-TP13 & RL-TP13 \\
\hline Central Plateau Office Facilities & RL-TP13 & & & & & & RL-113 & RL-TP13 & RL-IP13 \\
\hline Central Piateau Electrical Svstem & RL-TP13 & & & & & & RL- 1114 & RL-TP13 & RL-TP13 \\
\hline Central Plateau Liquid Sanitary Waste System & RL-TP13 & & & & & & RL-1113 & RL-TP13 & BL-TP13 \\
\hline Central Plateau Telecommuncation System & RL-TP13 & & & & & & RL-16 & RL-TP13 & RL-TP13 \\
\hline Central Plateau Shop Facilities & RL-TP13 & & & & & & $\mathrm{RL}=114$ & RL-TP13 & RL-TP13 \\
\hline Central Plateau Storage Support Facilities & RL-TP13 & & & & & & RL-17 & RL-TP13 & RL-TP13 \\
\hline Central Plateau Land & RL-TP13 & & & & & & $R L=1123$ & RL-TP13 & AL-TP13 \\
\hline Central Plateau Road System & RL-TP13 & & & & & & RL-S121 & RL-TP13 & Al-TP13 \\
\hline Central Plateau Rail System. & RL-TP13 & & & & & & RL-J122 & RL-TP13 & RL-TP13 \\
\hline Central Core Electrical System & RL-TP13 & & & & & & RL-1114 & RL-TP13 & RL-TP13 \\
\hline Central Core Water System & RL-TP13 & & & & & & RL-1112 & RL-TP13 & RL-TP13 \\
\hline Central Core Office Facilities & RL-TP13 & & & & & & RL-193 & RL-TP13 & RL-TP13 \\
\hline Central Core Solid Sanitary Waste System & RL-TP13 & & & & & & RL-1123 & RL-TP13 & RL-TP13 \\
\hline Central Core Liquid Sanitary Wasle System & RL-TP13 & & & & & & RL-S113 & RL-TP13 & RL-TP13 \\
\hline Central Core Telecommunication System & RL-TP13 & & & & & & RL. 16 & RL-TP13 & RL-TP13 \\
\hline Central Core Storage Facilities & RL-TP13 & & & & & & RL-17 & RL-TP13 & RL-TP13 \\
\hline Central Core Land & RL-TP13 & & & & & & AL-1123 & RL-TP13 & RL-TP13 \\
\hline Central Core Road System & RL-TP13 & & & & & & RL-1121 & RL-TP13 & RL-TP13 \\
\hline Central Core Rail System & RL-TP13 & & & & & & RL-S122 & RL-TP13 & RL-TP13 \\
\hline South 600 Area Electrical System & RL-TP13 & & & & & & RL-1114 & RL-TP13 & RL-TP13 \\
\hline South 600 Area Stearn System & RL-TP13 & & & & & & $\begin{array}{l}\text { RL-I111 } \\
\text { RL-TP14 }\end{array}$ & $\begin{array}{l}\text { RL-TP13 } \\
\text { RL-TP14 }\end{array}$ & $\begin{array}{l}\text { RL-TP13 } \\
\text { RL-TP14 }\end{array}$ \\
\hline South 600 Area Waler System & RL-TPt3 & & & & & & $\begin{array}{ll}\text { RL-I112 } \\
\text { RL-JP14 }\end{array}$ & $\begin{array}{l}\text { RL-TP13 } \\
\text { RL-TP14 }\end{array}$ & \begin{tabular}{|l|} 
RL-TP13 \\
RL-TP14 \\
\end{tabular} \\
\hline South 600 Area Liquid Sanitary Waste System & RL-TP13 & & & & & & $\begin{array}{l}\text { RL-J113 } \\
\text { RL-TP14 }\end{array}$ & $\begin{array}{l}\text { RL-TP13 } \\
\text { RL-TP14 }\end{array}$ & $\begin{array}{l}\text { RL-TP13 } \\
\text { RL-TP14 }\end{array}$ \\
\hline South 600 Area Telecommunication System & RL-TP13 & & & & & & $\begin{array}{l}\text { RL-16 } \\
\text { RL-TP14 }\end{array}$ & $\begin{array}{l}\text { RL-TP13 } \\
\text { RL-TP14 }\end{array}$ & $\begin{array}{l}\text { RL-TP13 } \\
\text { RL-TP14 }\end{array}$ \\
\hline South 600 Area Otfice Facilities & RL-TP13 & & & & & & $\begin{array}{l}\text { RL-I13 } \\
\text { RL-TP14 }\end{array}$ & $\begin{array}{l}\text { RL-TP13 } \\
\text { RL-TP14 }\end{array}$ & $\begin{array}{l}\text { RL-TP13 } \\
\text { RL-TP14 }\end{array}$ \\
\hline South 600 Area Shop Facilities & RL-TP13 & & & & & & $\begin{array}{l}\text { RL-11 } \\
\text { RL-TP14 }\end{array}$ & $\begin{array}{l}\text { RL-TP13 } \\
\text { RL-TP14 }\end{array}$ & $\begin{array}{l}\text { RL-TP13 } \\
\text { RL-TP14 }\end{array}$ \\
\hline South 600 Area Storage Facilities & RL.TP13 & & & & & & $\mid \begin{array}{l}\text { RL-17 } \\
\text { RL-TP14 }\end{array}$ & $\begin{array}{l}\text { RL-TP13 } \\
\text { RL-TP14 }\end{array}$ & $\begin{array}{l}\text { RL-TP13 } \\
\text { RL-TP14 }\end{array}$ \\
\hline South 600 Area Land & RL-TP13 & & & & & & $\begin{array}{l}\text { RL- } 1123 \\
\text { RL-TP14 } \\
\end{array}$ & $\begin{array}{l}\text { RL-TP13 } \\
\text { RL-TP14 }\end{array}$ & $\begin{array}{l}\text { RL-TP13 } \\
\text { RL-TP14 }\end{array}$ \\
\hline South 600 Area Road System & RL-TP13 & & & & & & $\begin{array}{l}\text { RL-I121 } \\
\text { RL-TP14 }\end{array}$ & $\begin{array}{l}\text { RL-TP13 } \\
\text { BL-TP14 } \\
\end{array}$ & $\begin{array}{l}\text { RL-TP13 } \\
\text { RL-TP14 }\end{array}$ \\
\hline
\end{tabular}




\begin{tabular}{|c|c|c|c|c|c|c|c|c|c|}
\hline \multirow{3}{*}{ Asset } & \multicolumn{9}{|c|}{ Lifo oycle } \\
\hline & \multirow{2}{*}{$\begin{array}{l}\text { Program } \\
\text { Planning }\end{array}$} & \multirow{2}{*}{$\begin{array}{c}\text { Pre } \\
\text { Conceptua }\end{array}$} & \multirow[b]{2}{*}{ Conceptual } & \multicolumn{3}{|c|}{ Execute } & \multirow[b]{2}{*}{ O\&M } & \multicolumn{2}{|c|}{ Close Out } \\
\hline & & & & Design & Construction & Tumover & & Post Ops & D\&D \\
\hline South 600 Area Rail System & RL-TP13 & & & & & & $\begin{array}{l}\text { RL-1122 } \\
\text { RL-TP14 }\end{array}$ & $\begin{array}{l}\text { RL-TP13 } \\
\text { RL-TP14 }\end{array}$ & $\begin{array}{l}\text { RL-TP13 } \\
\text { RL-TP14 }\end{array}$ \\
\hline
\end{tabular}

RL-I1 - DynCorp Infrastructure

RL-1111 - Steam Utilitites

RL-11 12 - Water Utilities

RL-J113 - Liquid Sanitary Waste Utilities

RL.1114 - Electrical Utilities

RL-1121 - Roadway Maintenance

RL-1122 - Rait Operations \& Maintenance

RL-1123 - Vehicle Operations

RL.113 - General Purpose Facilities

RL-114 - Infrastructure Services

RL-16 - Information Resource Management

RL-17 - Asset Management

RL-TP13 - Landlord

RL-TP14 - Hantord Surpius Facility Program 300 Area Revitalization Project

RL-WM05 - Liquid Ettluents Project 


\section{A.1.4 Project Planning Assumptions}

This section contains the issues that affect the project. These include project specific issues, as well as the site-fevel issues that have been assigned to the project for resolution. It also contains the assumptions that are used as a basis for the development of project plans until the issues are formally resolved with records of decision. The "Champion" column determines if the Project has lead responsibility or is an affected participant. If the champion belongs to the Project, the Project has the lead. If not, the Project is an affected participant. Project plans include appropriate activities and resources for resolving these issues.

Table A.1-3 Project Issues And Assumptions

\begin{tabular}{|c|l|c|c|}
\hline \multicolumn{1}{|c|}{ ISSUE } & PLANNING ASSUMPTION & CHAMPION \\
\hline 1 & $\begin{array}{l}\text { Revitalization Project Facility Timing } \\
\text { What is the acceptance criteria and timing for the transfer of } \\
\text { facilities in the } 300 \text { Area to the Revitalization Proiect? }\end{array}$ & & \\
\hline 2 & $\begin{array}{l}\text { Revitalization Project Facility Acquisition } \\
\text { Which facilities in the } 300 \text { Area will be transfered to the } \\
\text { Revitalization Project? }\end{array}$ & & \\
\hline
\end{tabular}




\section{A.2.0 Project Hanford Breakdown Structure Hierarchy}

The Project Hanford Breakdown Structure (PHBS) identifies the EM Project Hierarchy. This section identifies the. EM Projects within the PHBS specific to this Project.

\section{A.2.1 PHBS Hierarchy}

This is a diagram of the projects that are contained in this MYWP. There is a subsequent section for each of the projects contained in this hierarchy.

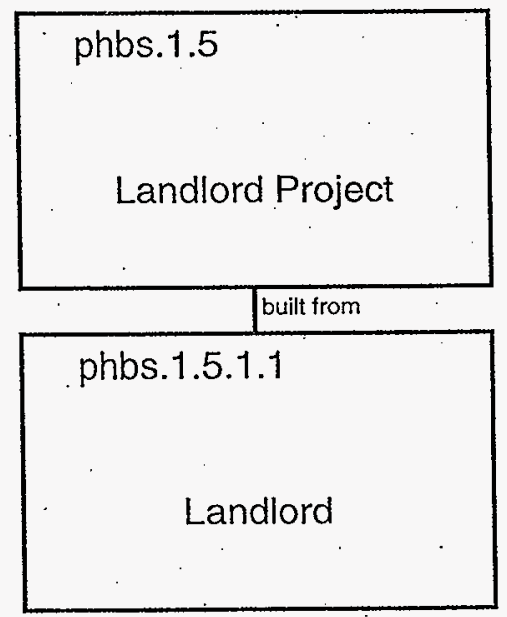

Figure A-1 Landlord Project 
3.0 SCHEDULE BASELINE 


\section{LANDLORD PROJECT \\ WBS 1.5}

FY 1998 Work Plan

HNF-SP-1242, Rev. 0

\section{A.3.1 Project SMBS by PBS}

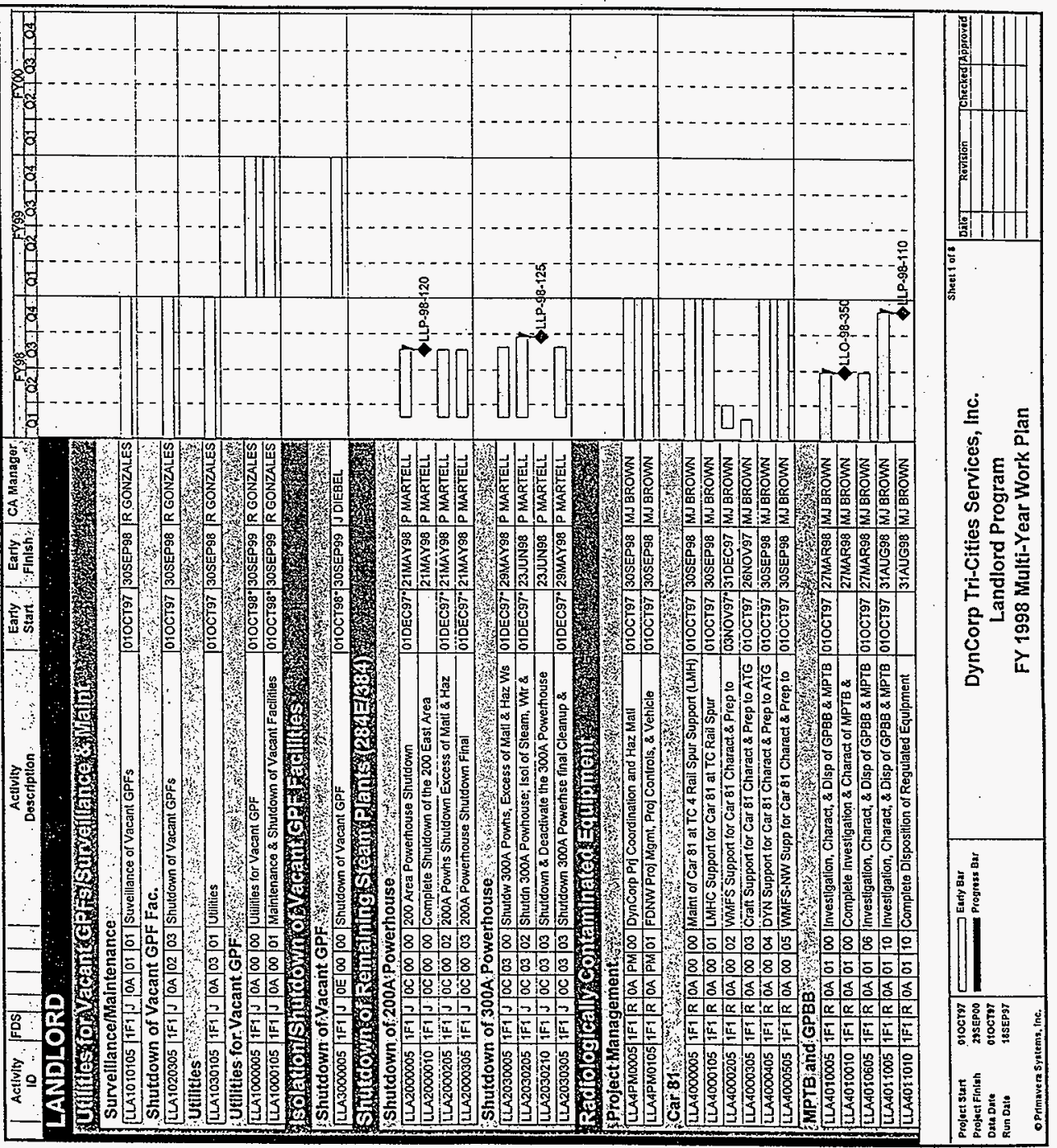




\section{A.3.1 Project SMBS by PBS}

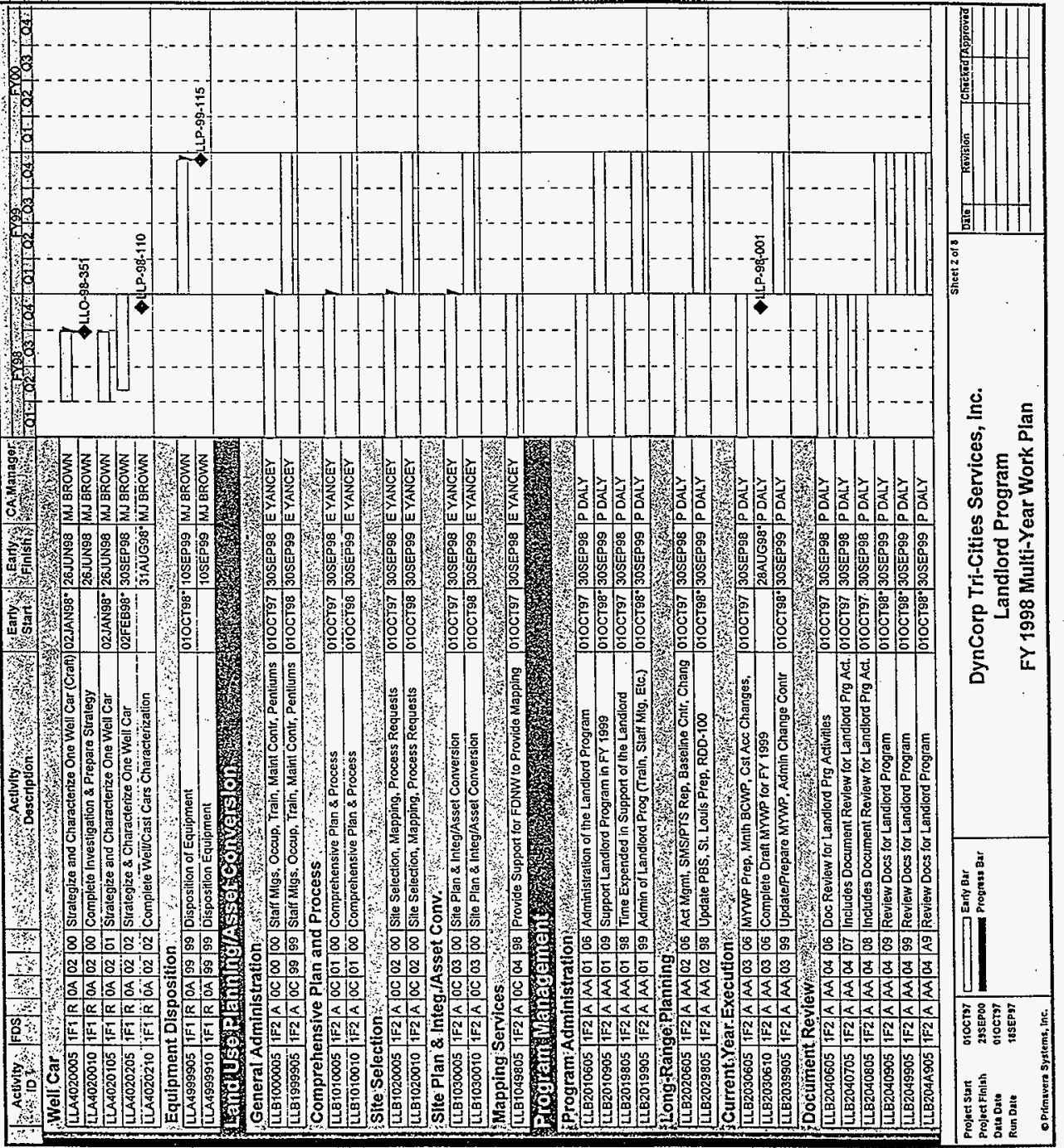




\section{A.3.1 Project SMBS by PBS}

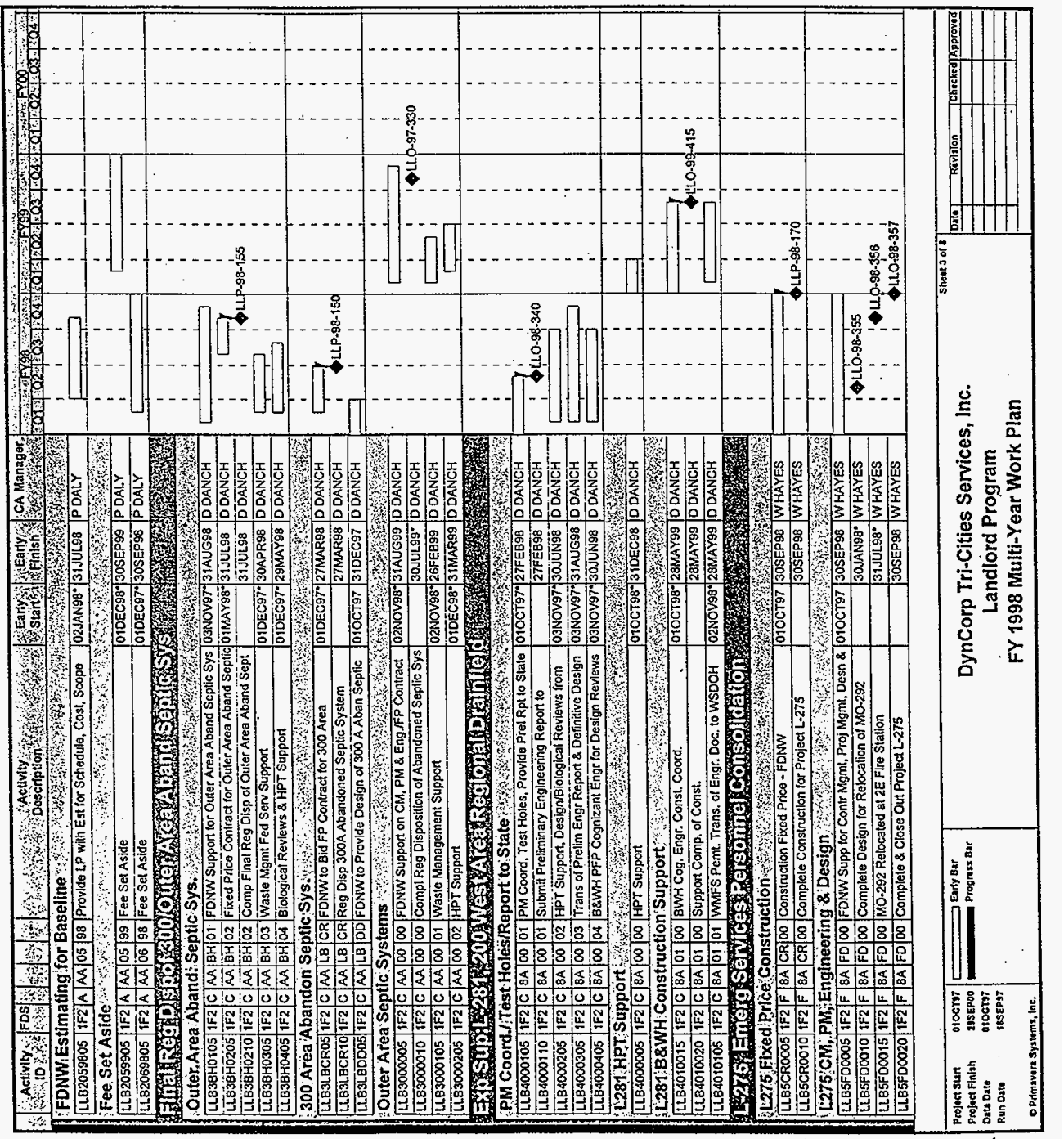




\section{A.3.1 Project SMBS by PBS}

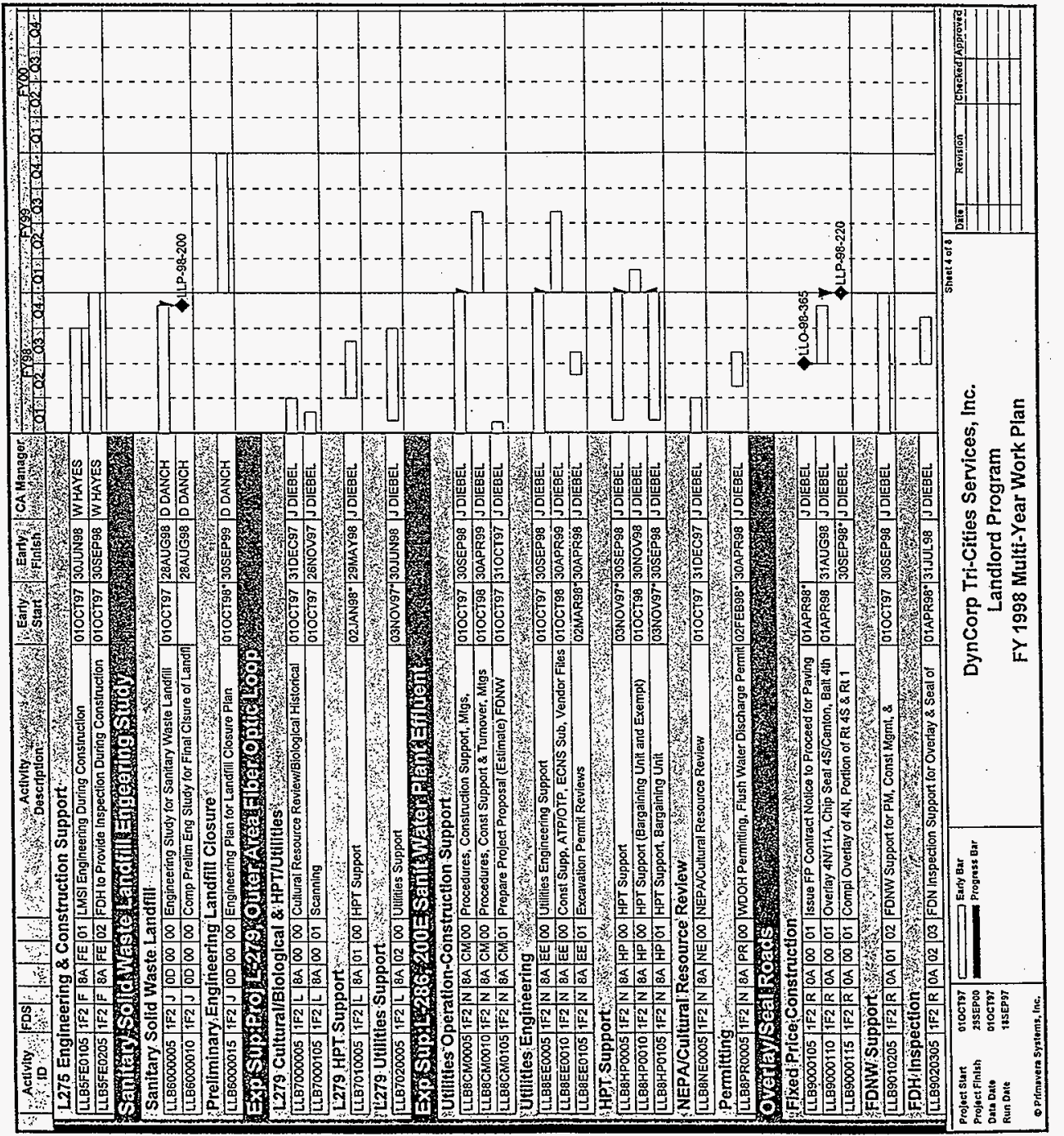




\section{A.3.1 Project SMBS by PBS}

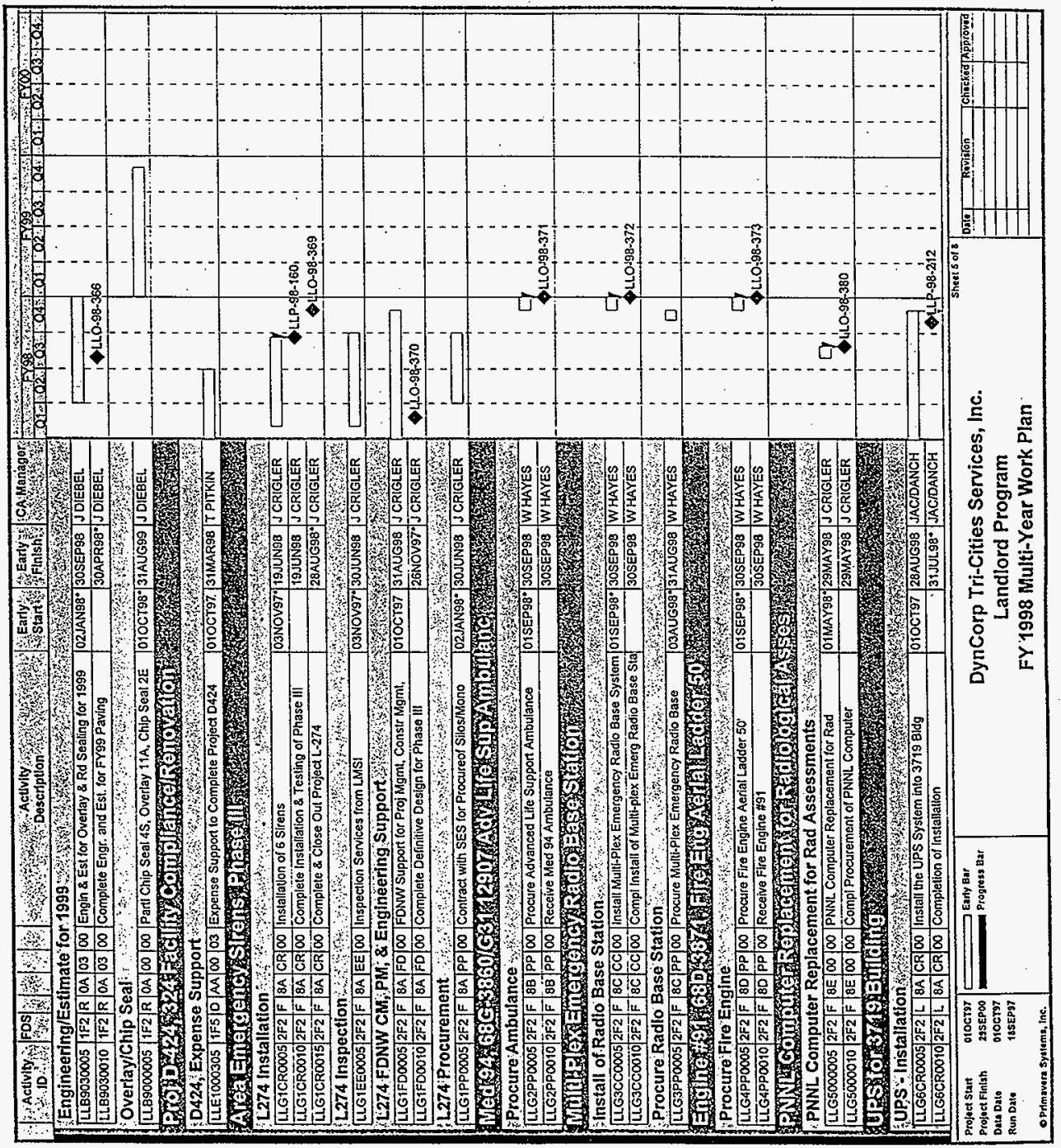




\section{A.3.1 Project SMBS by PBS}

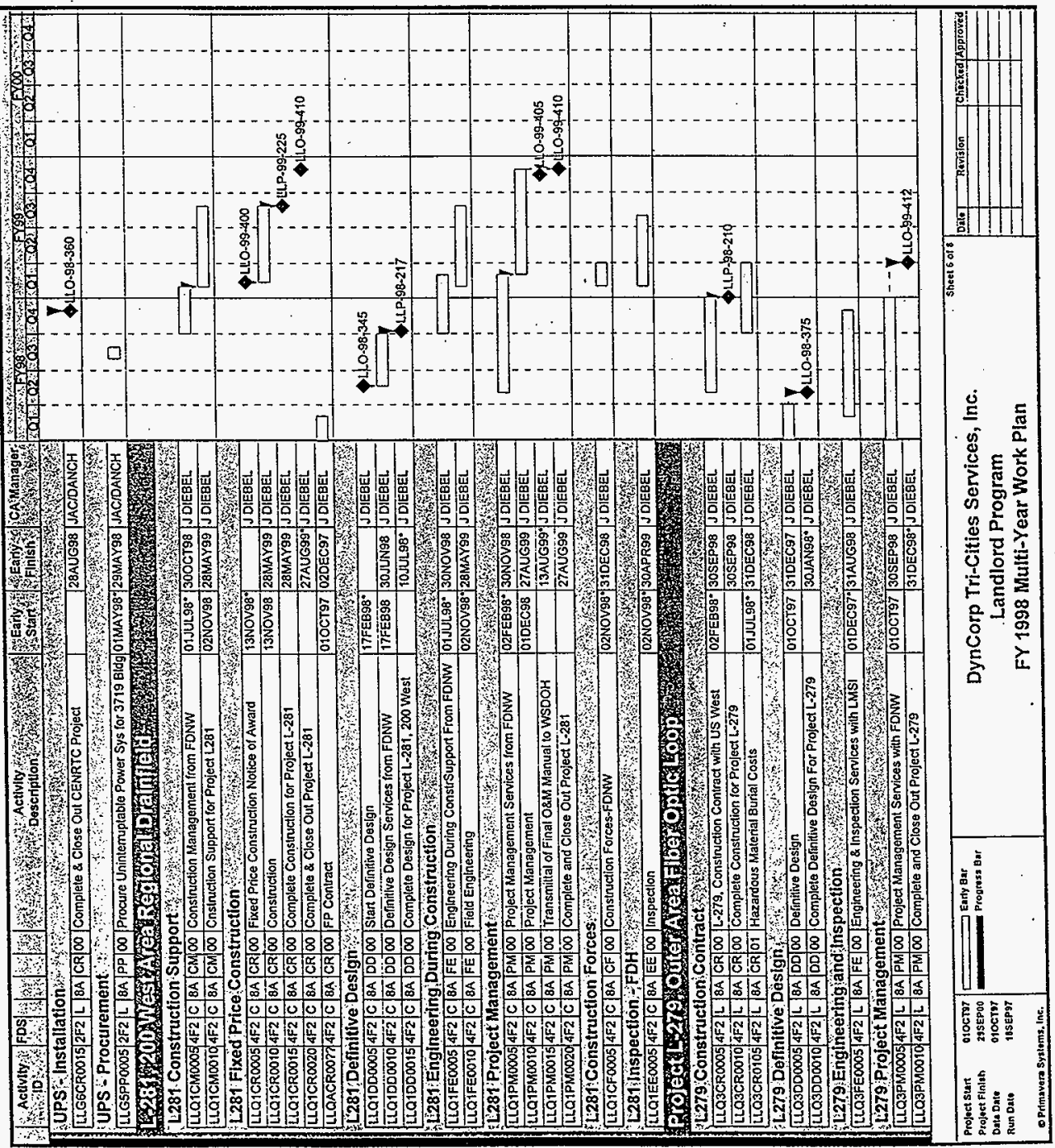




\section{A.3.1 Project SMBS by PBS}

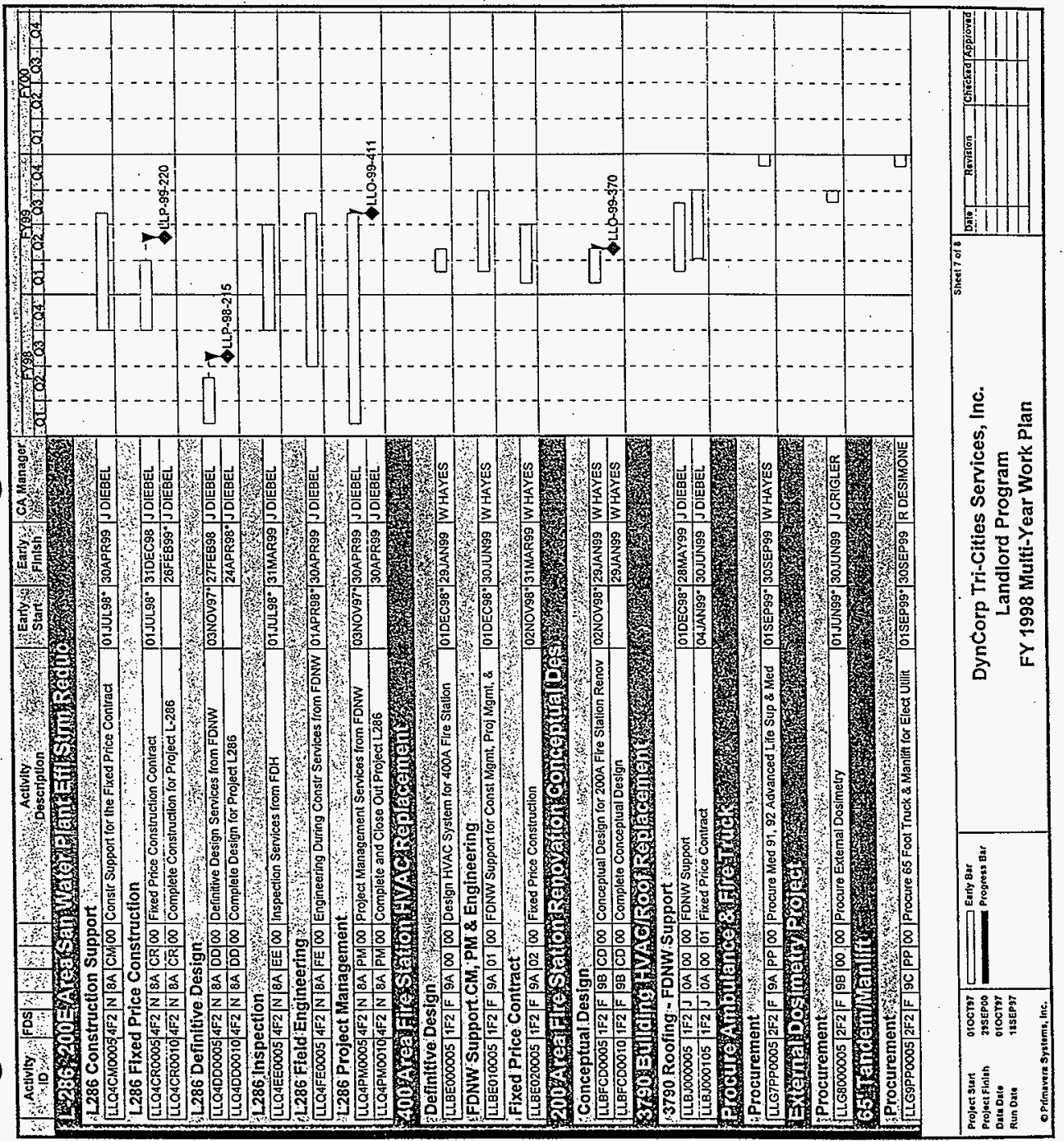




\section{LANDLORD PROJECT \\ WBS 1.5}

FY 1998 Work Plan

HNF-SP-1242, Rev. 0

\section{A.3.1 Project SMBS by PBS}

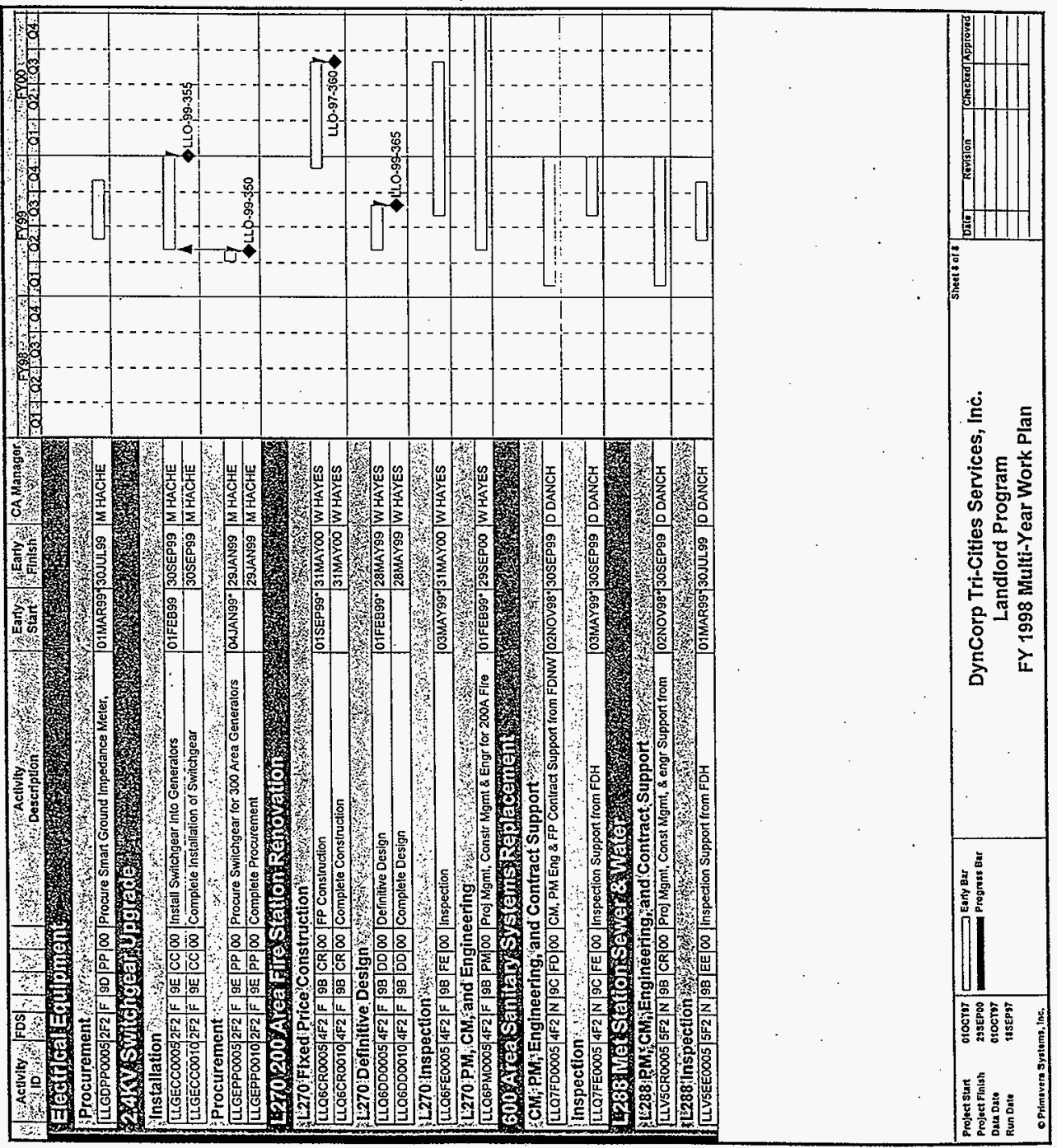




\section{LANDLORD PROJECT \\ WBS 1.5}

FY 1998 Work Plan

HNF-SP-1242, Rev. 0

\section{A.4.1 Basis of Estimate}

The Landlord Project's cost estimates were prepared in accordance with HNF-PR-000585, Cost Estimating. The estimates utilize the Activity-based Costing (ABC) method in all cases. These estimates are based on a "bottoms up" approach to determine: 1) resources necessary to complete each discrete piece of workscope; 2) how the resources are allocated by year and by month; 3) priorities based on DOE-RL, DOE-HQ, Stakeholder and Contractor values of cleanup work to be completed and its applicability to the current priority structure and the overall site mission.

Cost estimates for FY 1998 and 1999 were prepared at the task level. Cost estimates for FY 2000 were prepared at the cost account level with cost estimates for FY 2001 through FY 2006 at the activity level. For the remainder of the project life cycle, estimates have been prepared at the end function level.

The cost estimating packages contain the following information:

- Technical scope

- Purpose of the workscope to be completed

- Assumptions and exclusions

- Estimate work breakdown structure

- Technique and historical basis

- Cost estimating relationship development

- Labor rates, unit rates, and unit cost sources

- Escalation amounts and sources

- Contingency calculation development (if applicable)

- Legal drivers

- Labor costs

- Technical logic diagram

- Integrated, logic driven, resource loaded schedules

- Responsibility assignment matrix

- Other costs that will be reflected in the budget for the particular project.

The activity-based cost estimates can be found in our scheduling office at 2430 Stevens Drive, Room 252. 
LANDLORD PROJECT

SUMMARY OF LIFE CYCLE COST BASELINE (BCWS) BY YEAR BY BY PROJECT BASELINE SUMMARY (PBS)

FY 1998

(\$000s)

\begin{tabular}{|c|c|c|c|c|c|c|c|c|c|c|c|c|}
\hline & \multirow{2}{*}{$\begin{array}{l}\text { SUBTOT } \\
\text { FY1997- } \\
\text { FY2006 }\end{array}$} \\
\hline PBS TITLE & PBS NO & FY1997 & \% & FY1999 & FY2000 & FY2001 & FY2002 & FY2003 & FY2004 & FY2005 & FY2006 & \\
\hline & & & & & & & & & & & & \\
\hline \begin{tabular}{|l} 
Landiord Ptoject \\
\end{tabular} & 13 & 11.037 & 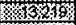 & 13,862 & 24,588 & 47,669 & 40,863 & 25,626 & 20.215 & 32,203 & 38,982 & 268,264 \\
\hline RL Contracts for Landlord & RL-TP13 & & $1 \times 593$ & & & & & & & & & 493 \\
\hline & & & m & & & & & & & & & $\cdot$ \\
\hline & & & 筮 & & & & & & & & & $\cdot$ \\
\hline & 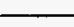 & 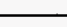 & (x) & 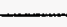 & {$[1$} & - & - & & $m$ & & & $\div$ \\
\hline & & & r. & & & & & & & & & $\therefore$ \\
\hline & & & 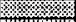 & & & & & & & & & $\rightarrow$ \\
\hline & & & & & & & & & & & & $\div$ \\
\hline & & & ক & & & & & & & & & $\therefore$ \\
\hline & & & \%, & & & & & & & & & -1 \\
\hline S & & ; & S & 3 & 243 & 40 & 箋 & 刑 & 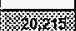 & (s) & 筮 & 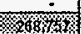 \\
\hline
\end{tabular}

'Budgeted Cost of Work Scheduled (BCWS) Equals Performance Measurement Baseline (PMB); Expense Carryover is NOT Included. 


\section{LANDLORD PROJECT}

SUMMARY, OF LIFE CYCLE COST BASELINE (BCWS) BY YEAR BY BY PROJECT BASELINE SUMMARY (PBS)

FY 1998

(\$000s)

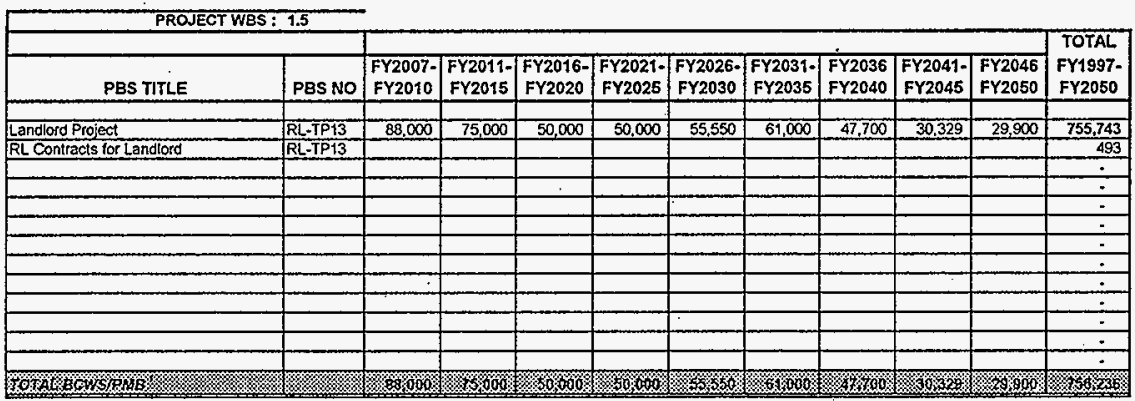


LANDLORD PROJECT

SUMMARY OF LIFE CYCLE BUDGET AUTHORITY (BIA) BY YEAR BY

BY PROJECT BASELINE SUMMARY (PBS)

FY 1998

(\$000s)

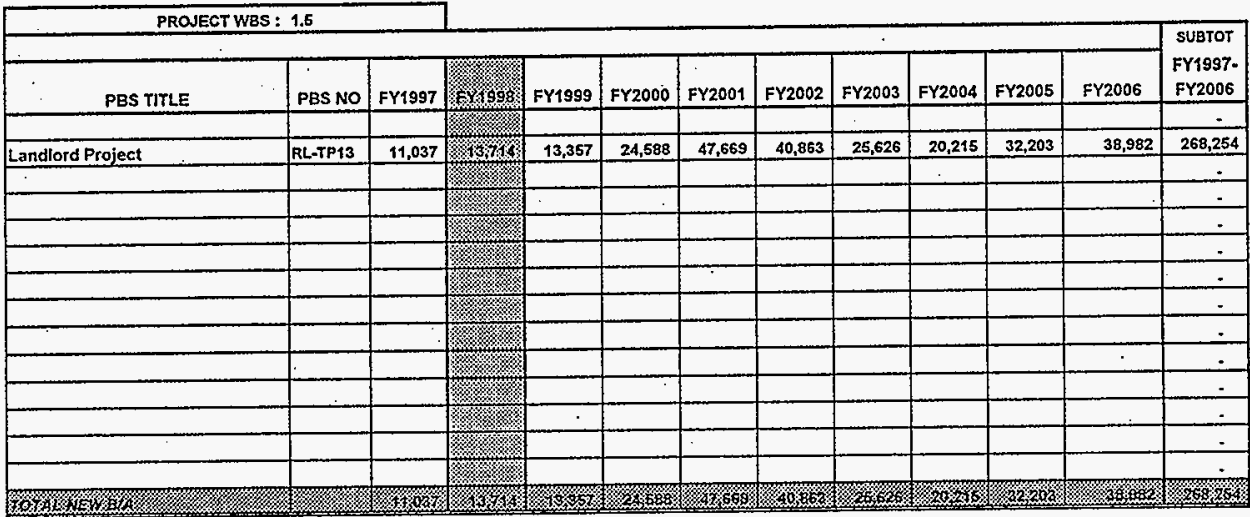


LANDLORD PROJECT

SUMMARY OF LIFE CYCLE BUDGET AUTHORITY (B/A) BYYEAR BY

BY PROJECT BASELINE SUMMARY (PBS)

FY 1998

(\$000s)

PROJECT WES : 1.5

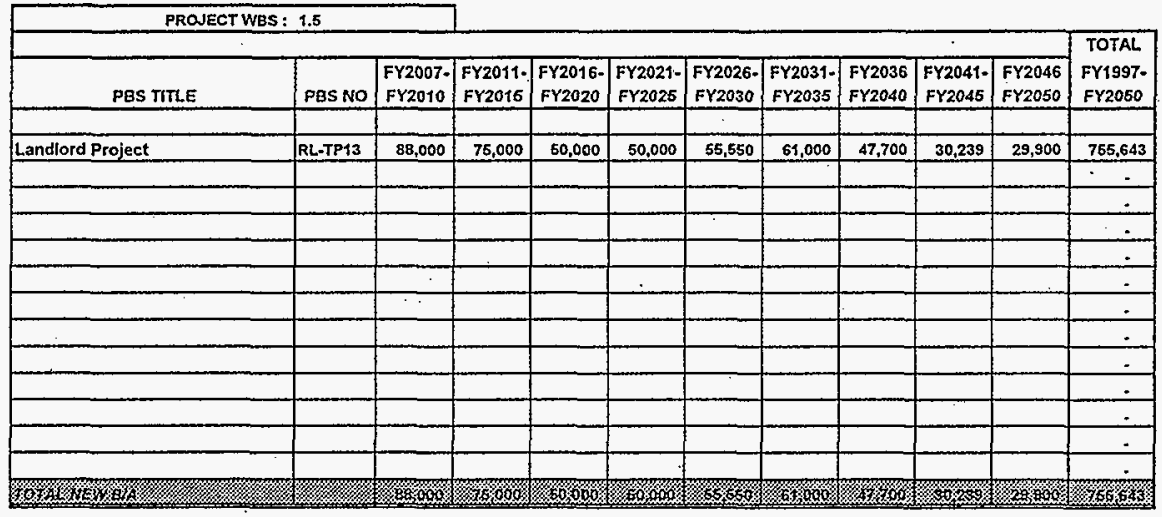




\section{LANDLORD PROJECT WBS 1.5}

FY 1998 Work Plan

HNF-SP-1242, Rev. 0

\section{A.4.4 BCWS for Execution Year by Month by Fund Type}

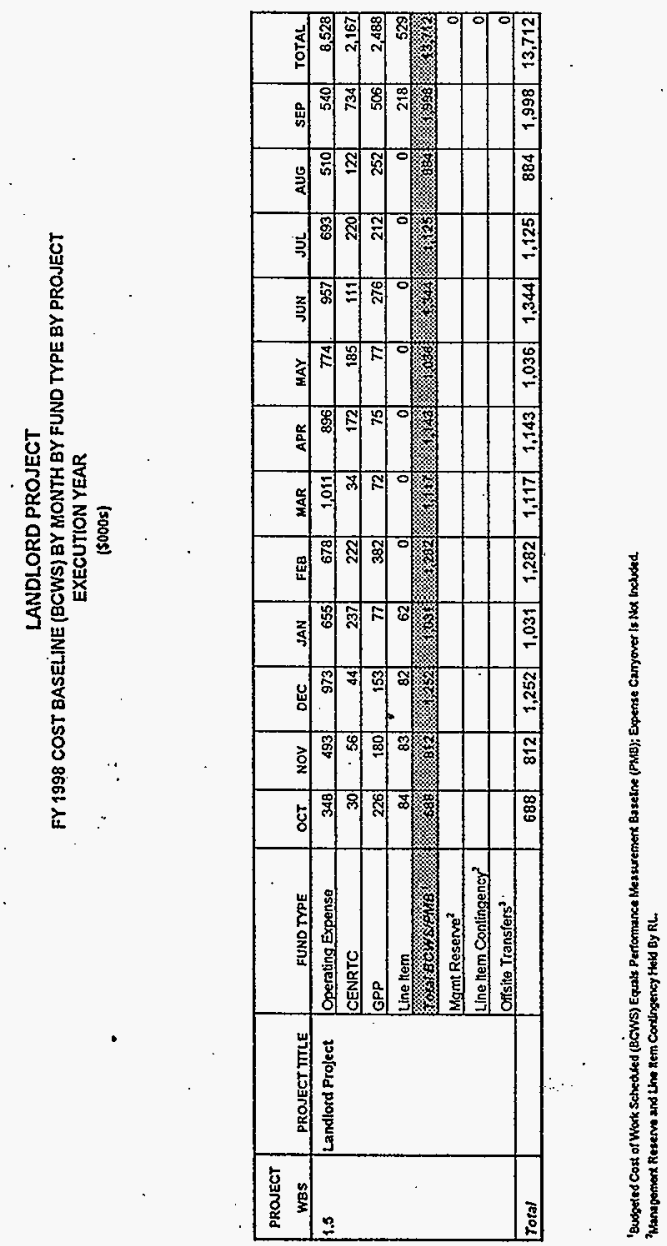


LANDLORD PROJECT

WBS 1.5

FX 1998 Work Plan

HNF-SP-1242, Rev. 0

\section{A.4.5 BCWS for Execution Year by Month by Cost Element}

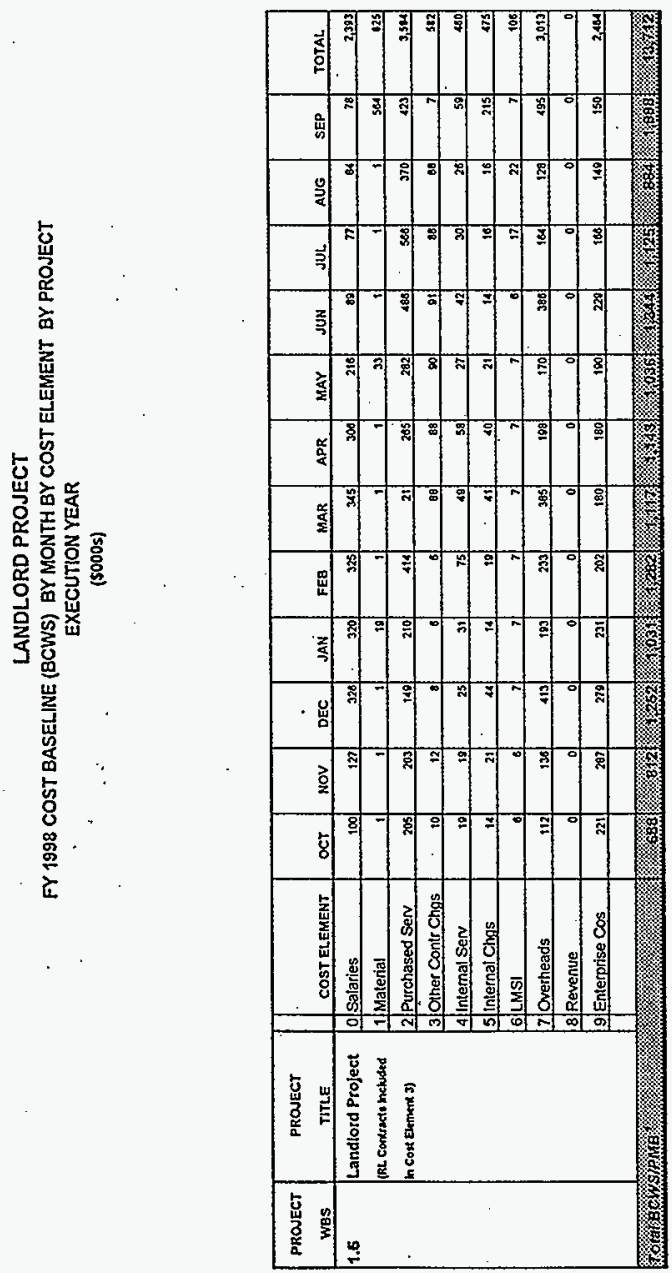

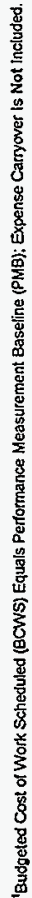


5.0 PERFORMANCE

MEASURES/METRICS 


\section{LANDLORD PROJECT}

WBS 1.5

FY 1998 Work Plan

HINF-SP-1242, Rev. 0

\section{A.5.0 Performance Measures/Metrics Including Cost Metrics}

Not applicable. 


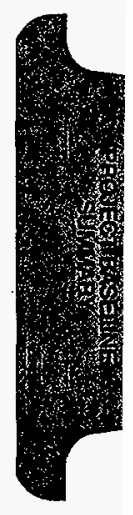




\section{B.1 Landlord (RL-TP13)}

\section{B.1.1.0 Landlord Technical Baseline (RL-TP13)}

\section{B.1.1.1 Landlord Organization Mission (RL-TP13)}

The mission of Landlord Project is to preserve, upgrade, maintain, and forecast cost effective general infrastructure activities to facilitate the Hanford Site cleanup mission. Specific functions and services provided by Landlord Project include utilities (i.e. steam, water, sanitary sewer, solid waste disposal, electrical and telecommunication distribution), transportation, general purpose facilities (includes general support shops and laboratories), services, and energy and land use management. All Landlord Project activities will be performed in an environmentally sound, safe, economical, prudent, and reliable manner. The Hanford Site Landlord Project will be competitive with commercially provided services to offer the best price, quality, and service available.

The major project objectives are:

The overall Landlord Project objective is to maintain a standard of excellence and safety while providing efficient, affordable, and effective infrastructure and support services.

The Project will strive to maintain, preserve, or upgrade strategic assets while consolidating facilities and functions to lower operating and maintenance costs. Realistic goals and strong baseline control are critical to meeting the Project objectives. Paramount to these objectives is compliance with all applicable laws, orders, agreements, codes, standards, and best management and safety practices.

The objectives for general infrastructure support are reflected in two specific areas, 1) Core Infrastructure Maintenance, and 2) Infrastructure Mortgage Reduction.

The following objectives are applicable to the Landlord Project:

Continue to focus on defining and addressing core infrastructure needs. Utilize mortgage reduction (i.e. elimination, excessing, salvage, and demolition) to transition facilities and equipment to their most cost effective status; thereby optimizing the site infrastructure. Provide upgrades to the Site only where cost effective.

Provide an infrastructure that serves the Hanford Mission, leaves diversification value and viable assets, concentrate service locations, excess, disposition, or demolish inadequate and under utilized facilities, promote energy efficiency, and maintain facilities and equipment as economicall and safely as possible within environmental regulations.

Integrate Occupational Safety and Health Act (OSHA) requirements into the continuous facility inspection project. Ensure compliance with applicable federal and state regulations, and the Tri-Party Agreement.

Identily future infrastructure needs, integrate Landlord Project planning with other project plans, effectively use limited resources, improve project definition and cost estimating, and direct fund all appropriate costs.

Develop a reliable and meaningful process for performing inspections, reporting and life cycle costing of projects for Landlord functions ensuring effective asset management of site resources.

Encourage outreach and partnership with local/private sector and Tribal Governments. 
Provide site wide coordination and management of land use issues to ensure effective support to site cleanup decisions and economic transition initiatives.

\section{B.1.1.2 Landlord End Point Targets from Hanford Strategic Plan}

\section{B.1.1.2.1 Landlord Principal End Point Targets}

- Transition high cost suplus facilities in the central plateau and south 600 areas to a low cost, stable, deactivated condition.

- Remove non-essential, surplus buildings and facilities that don't have identified post-cleanup uses.

- Dismantle, or close through entombment, D\&D facilities in the central plateua areas currently assigned to the ER program.

* Reuse facilities in the south 600 area for economic diversification where feasible.

\section{B.1.1:2.2 Landlord Supported End Point Targets}

-None- 
2.0 WORK BREAKDOWN

STRUCTURE 


\section{LANDLORD PROJECT \\ WBS 1.5}

\section{B.2.1 Work Breakdown Structure}

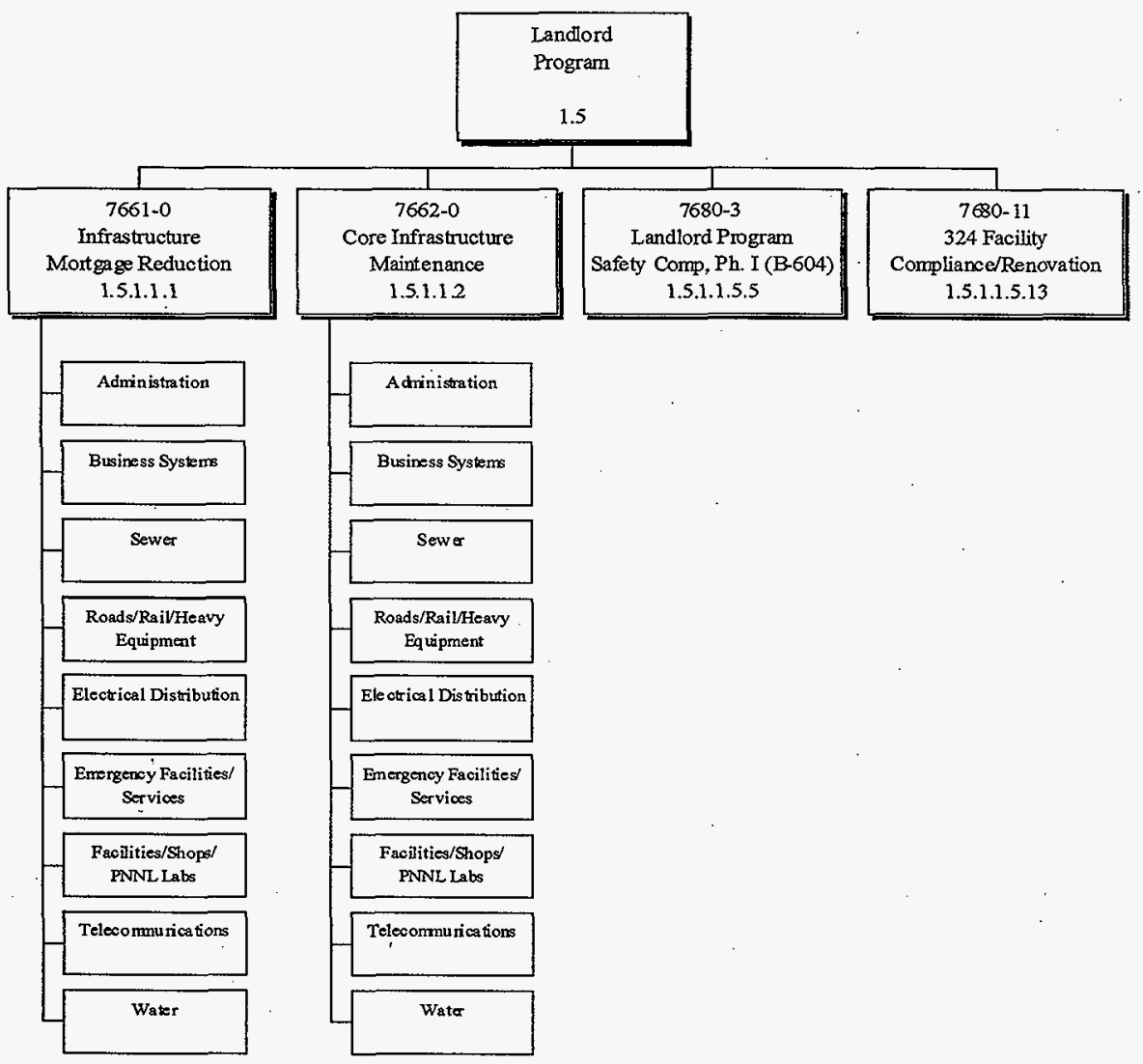




\section{LANDLORD PROJECT \\ WBS 1.5}

FY 1998 Work Plan

HNF-SP-1242, Rev. 0

\section{B.2.2 WBS Dictionary}

\section{HANFORD SITE WORK BREAKDOWN STRUCTURE DICTIONARY LANDLORD}

WBS: 1.5

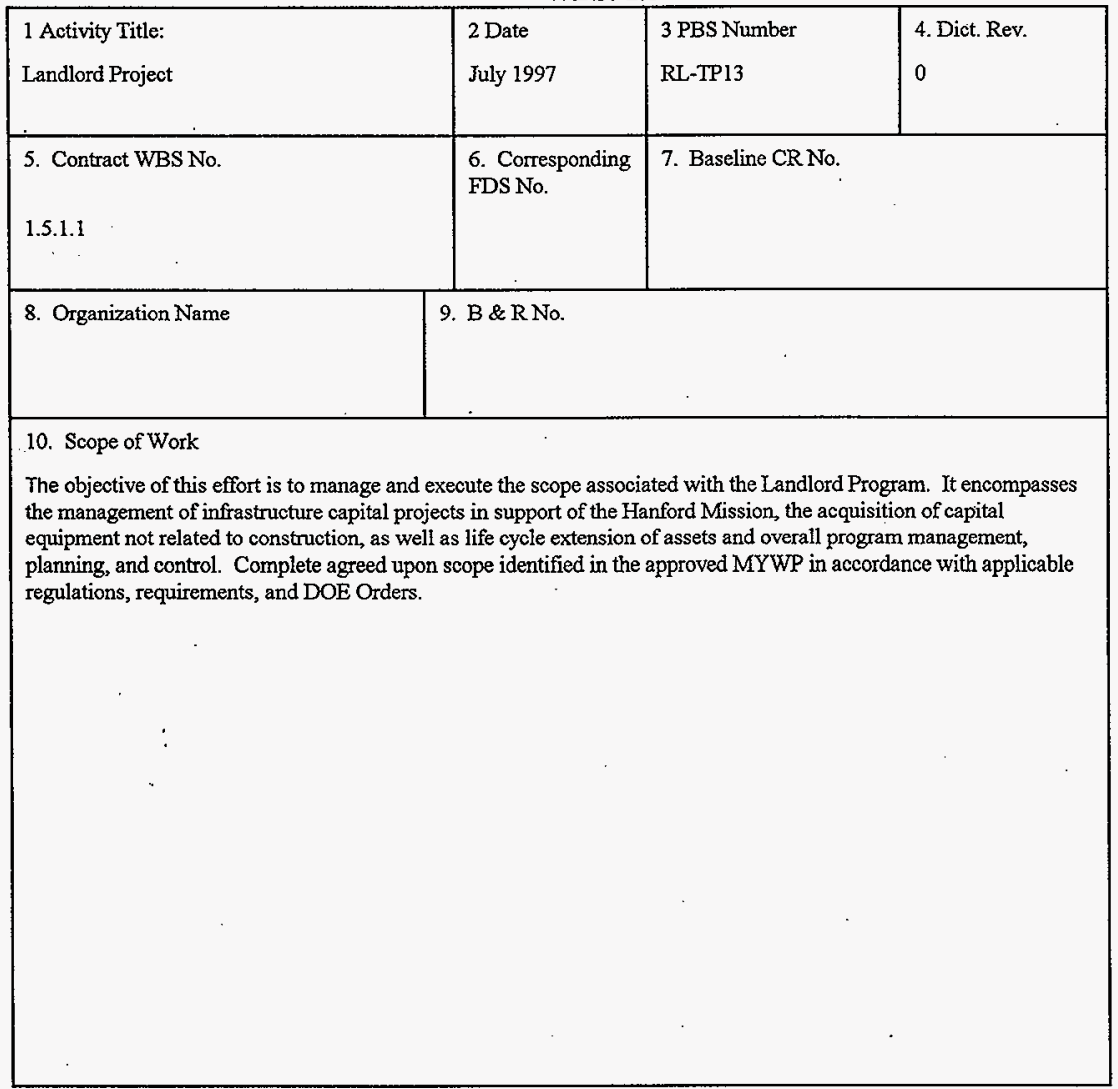




\section{HANFORD SITE WORK BREAKDOWN STRUCTURE DICTIONARY LANDLORD}

WBS: 1.5

\begin{tabular}{|l|l|l|c|}
\hline $\begin{array}{l}\text { 1. Activity Title: } \\
\text { Infrastructure Mortgage Reduction }\end{array}$ & $\begin{array}{l}\text { 2 Date } \\
\text { July } 1997\end{array}$ & $\begin{array}{l}\text { 3. PBS Number } \\
\text { RL-TP13 }\end{array}$ & $\begin{array}{c}\text { 4. Dict. Rev. } \\
0\end{array}$ \\
\hline 5. Contract WBS No. & $\begin{array}{l}\text { 6. Corresponding } \\
\text { FDS No. }\end{array}$ & 7. Baseline CR No. \\
\hline $\begin{array}{l}\text { 8. Organization Name } \\
\text { DynCorp Operations }\end{array}$ & $\begin{array}{l}\text { 9. B \& RNo. } \\
\text { EW7070201 }\end{array}$ & \\
\hline 10. Scope of Work & & \\
\hline
\end{tabular}

Transition the Hanford Site infrastructure with the goal to reduce outyear cost. The activity includes the following: reutilization of vacant land and properties; surveillance, maintenance, and demolition of vacant general purpose facilities (no funding in either FY 1998 or 1999); transfer of retired facilities (steam plants, electrical distribution systems, etc.) to other programs or agencies; disposition of contaminated equipment (railroad cars, cranes, vehicles, etc.); and surveillance, maintenance, and demolition of abandoned infrastructure systems (pump stations, electrical substations, water and sewer systems, etc. No funding is included for any demolition in either FY 1998 or 1999). 


\section{HANFORD SITE WORK BREAKDOWN STRUCTURE DICTIONARY LANDLORD}

WBS: 1.5

\begin{tabular}{|l|l|l|c|}
\hline $\begin{array}{l}\text { 1. Activity Title: } \\
\text { Core Infrastructure Maintenance }\end{array}$ & $\begin{array}{l}\text { 2Date } \\
\text { July } 1997\end{array}$ & $\begin{array}{l}\text { 3. PBS Number } \\
\text { RL-TP13 }\end{array}$ & $\begin{array}{c}\text { 4. Dict. Rev. } \\
0\end{array}$ \\
\hline 5. Contract WBS No. & $\begin{array}{l}\text { 6. Corresponding } \\
\text { FDS No. }\end{array}$ & 7. Baseline CR No. \\
\hline $\begin{array}{l}\text { 8. Organization Name } \\
\text { DynCorp Operations }\end{array}$ & $\begin{array}{l}\text { 9. B \& R No. } \\
\text { EW7070201 }\end{array}$ & \\
\hline
\end{tabular}

10. Scope of Work

This activity provides for the maintenance and replacement of core infrastructure systems to extend the life of the infrastructure to support the site mission to completion. The activity includes the maintenance and replacement of the following: highways and roads; railroads; supply and distribution of water for domestic and fire protection purposes (primarily in the 200 and 100 areas) sanitary waste water collection, treatment, and disposal systems (majority are septic systems north of the Wye Barricade); telephone and telecommunication networks (includes emergency and industrial signals, Hanford Local Area Network); and primary electrical distribution systems. Replacement of systems will be performed to resolve safety, health, and/or environmental issues or when cost effective and where life cycle savings can be realized. The infrastructure is needed to support the overall mission of the Hanford Site which includes waste management, environmental cleanup, research, and development. 


\section{HANFORD SITE WORK BREAKDOWN STRUCTURE DICTIONARY LANDLORD}

WBS: 1.5

\begin{tabular}{|l|l|l|l|}
\hline $\begin{array}{l}\text { 1. Activity Title: } \\
\begin{array}{l}\text { Project L-035, (Subproject B-604) } \\
200 \text { Area Water System Upgr. - Reservoir }\end{array}\end{array}$ & $\begin{array}{l}\text { 3. Pate } \\
\text { July } 1997 \\
\text { RL-TP13 }\end{array}$ & $\begin{array}{c}\text { 4. Dict. Rev. } \\
0\end{array}$ \\
\hline 5. Contract WBS No. & $\begin{array}{l}\text { 6. Corresponding } \\
\text { FDS No. } \\
\text { 1.5.1.1.5.5 }\end{array}$ & $\begin{array}{l}\text { 7. Baseline CR No. } \\
\text { 8. Organization Name } \\
\text { DynCorp Operations }\end{array}$ & $\begin{array}{l}\text { 9. B \& R No. } \\
\text { EW7070201 }\end{array}$ \\
\hline
\end{tabular}

10. Scope of Work

This WBS of the Hanford Landlord Program consists of Project B-468, "Railroad Upgrade Mainline"; Project B-604, "Water System Upgrades-Reservoir"; Project B-690, "Steam System Safety and Productivity Upgrade"; and Project L-001, "Fire Water Storage and Distribution Upgrades, 300 Area." Subproject B-468 replaced rail between the 1100 and 200 Areas and from the Columbia Center to the Yakima River bridge and completed in FY 1995. Subproject B-604 upgrades the capacity of the current water storage and supply systems for the 200 Area to meet RLIP 5480.7 and the National Fire Protection Associated standards. Subproject B-690 replaced existing electrical service, circuits and components in the 44 year old steam generation facilities in the 200 Areas and was completed in FY 1994. Subproject L-001 upgraded the existing 300 Area water system as required to provide an independent water supply capable of meeting fire flow demands simultaneous with peak,process and domestic demands for a period of not less than four hours and completed in FY 1995. 


\section{HANFORD SITE WORK BREAKDOWN STRUCTURE DICTIONARY LANDLORD}

WBS: 1.5

\begin{tabular}{|l|l|l|c|}
\hline $\begin{array}{l}\text { 1. Activity Title: } \\
\begin{array}{l}\text { Project D-424, 324 Facility Compliance/ } \\
\text { Renovation }\end{array}\end{array}$ & $\begin{array}{l}2 \text { Date } \\
\text { July } 1997\end{array}$ & $\begin{array}{l}\text { 3. PBS Number } \\
\text { RL-TP13 }\end{array}$ & $\begin{array}{c}\text { 4. Dict. Rev. } \\
0\end{array}$ \\
\hline $\begin{array}{l}\text { 5. Contract WBS No. } \\
\text { 1.5.1.1.5.13 }\end{array}$ & $\begin{array}{l}\text { 6. Corresponding } \\
\text { FDS No. }\end{array}$ & 7. Baseline CR No. \\
\hline $\begin{array}{l}\text { 8. Organization Name } \\
\text { DynCorp Operations }\end{array}$ & $\begin{array}{l}\text { 9. B \& R No. } \\
\text { EW7070201 }\end{array}$ & \\
\hline
\end{tabular}

10. Scope of Work

This WBS of the Hanford Landlord Program consists of bringing selected 324 Building systems into compliance; avoiding near-term adverse impacts on workers, the public, and the environment; and assures continuity of operations to meet requirements of key Hanford programs. The deteriorated, contaminated, or essential noncompliant building systems in this project must be renovated in accordance with U.S. Department of Energy (DOE) Orders, federal codes, and state regulations. Operating costs reflect funding required for validation efforts . and direct non-dedicated support to the authorized Line Item project. The construction scope includes: replacing worn out and deteriorated electrical service equipment which are very old and difficult to maintain because key parts are no longer manufactured; replacing wiring and connections which violate the National Electric Code (NEC); installing seismic bracing, supports, couplings, standpipes, fire department connections, backflow preventors, and the expansion of the fire sprinkler system to meet National Fire Protection Act (NFPA) codes. This project has been descoped to complete only NEC, NFPA, and associated fire protection activities so the facility can be cleaned out and transitioned for demolition. 


\section{LANDLORD PROJECT \\ WBS 1.5}

FY 1998 Work Plan

HNF-SP-1242, Rev. 0

\section{B.2.3 Landlord Responsibility Matrix}

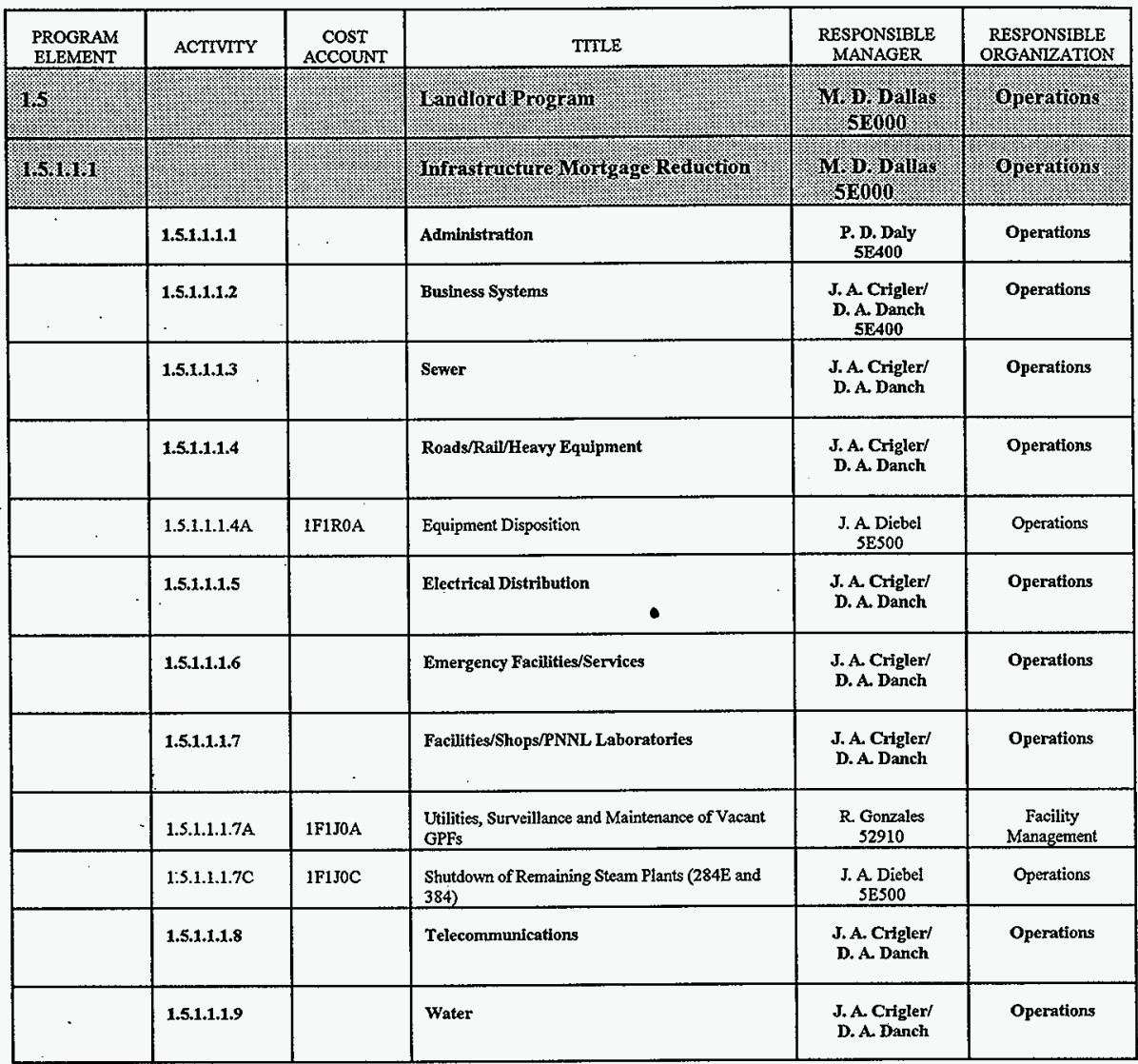




\section{LANDLORD PROJECT \\ WBS 1.5}

FY 1998 Work Plan

HNF-SP-1242, Rev. 0

\section{B.2.3 Landlord Responsibility Matrix}

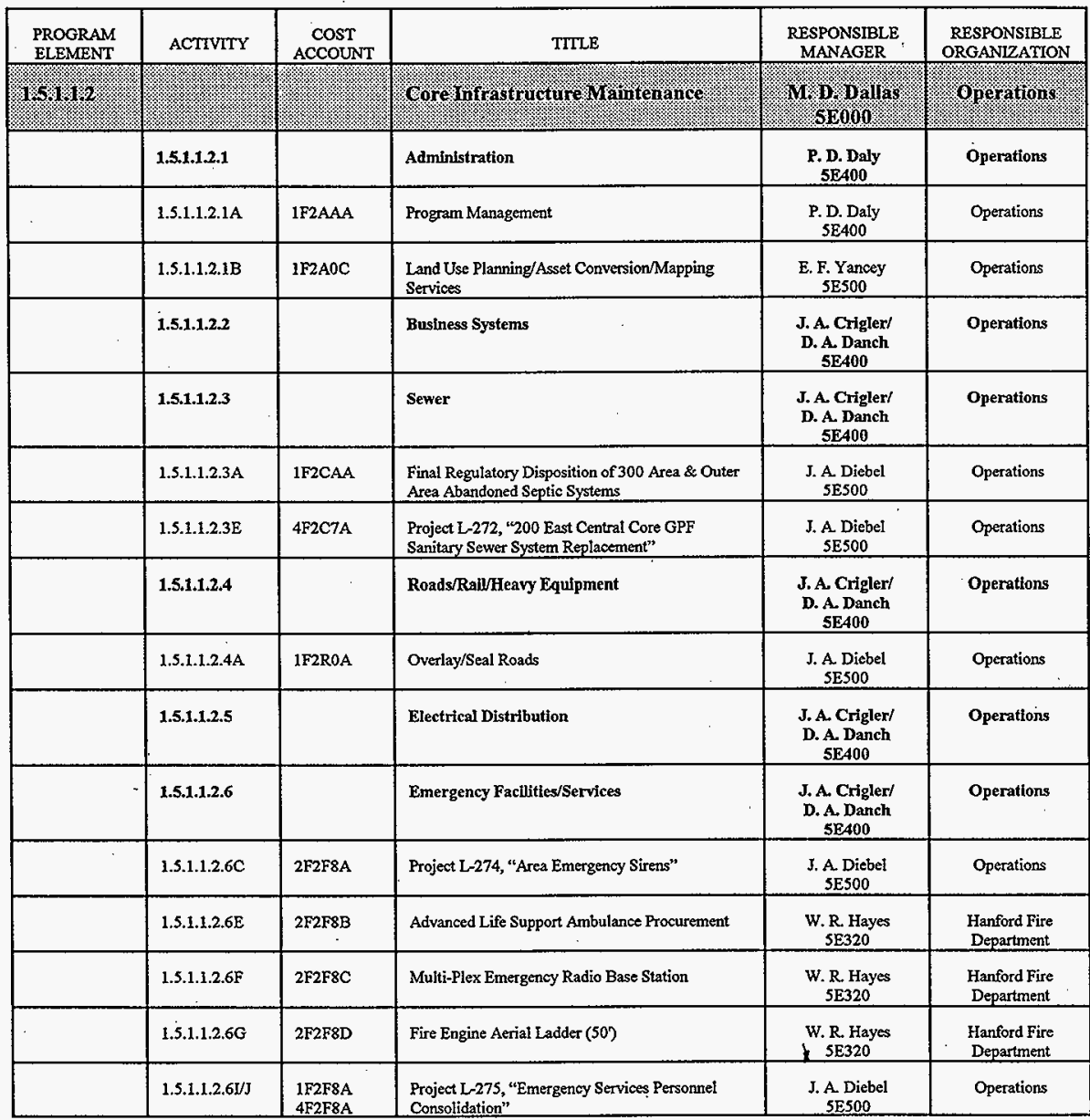




\section{LANDLORD PROJECT \\ WBS 1.5}

FY 1998 Work Plan

HNF-SP-1242, Rev. 0

\section{B.2.3 Landlord Responsibility Matrix}

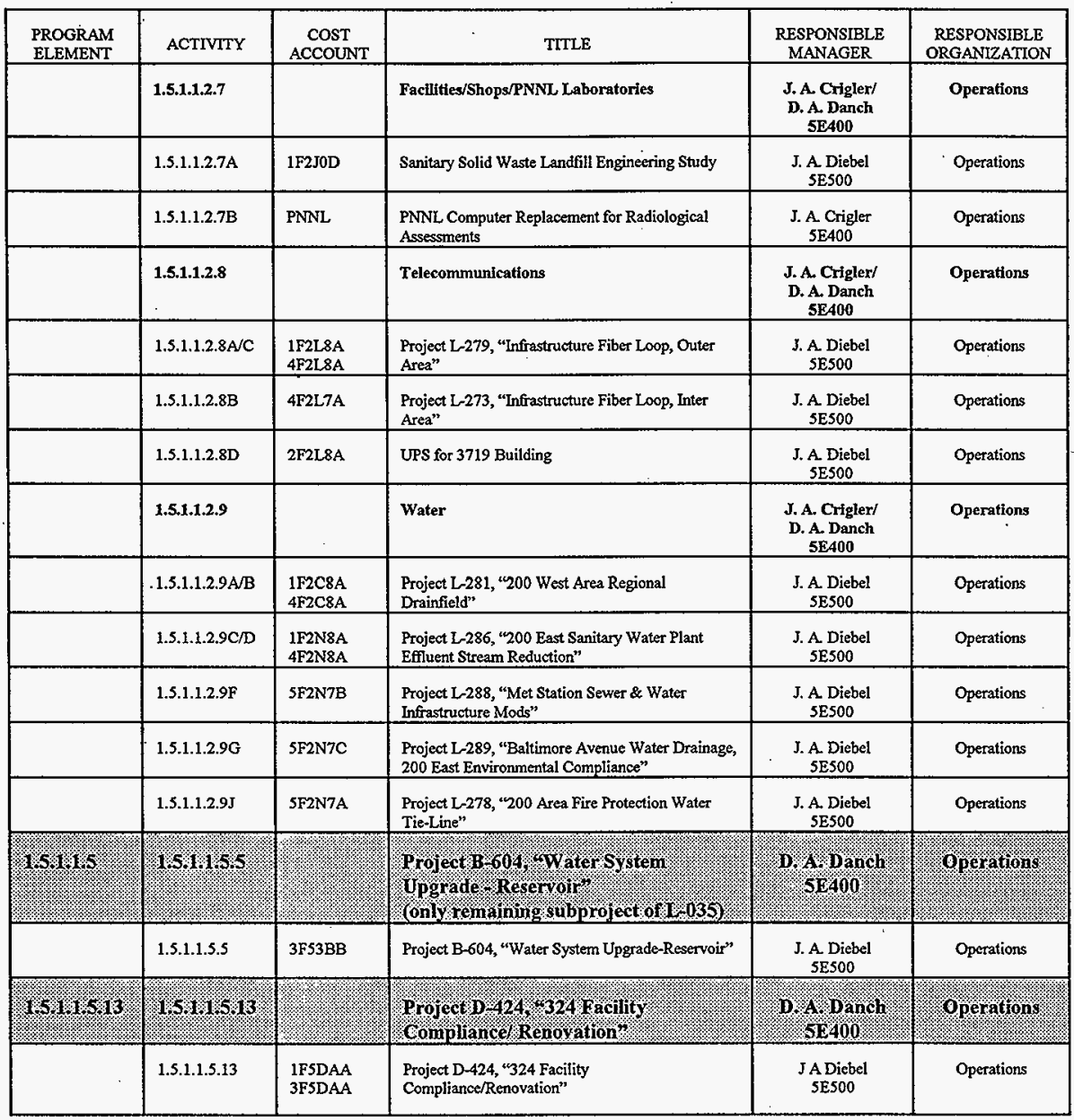




\section{B.3.1/B.3.2 PMBS by PBS/Execution Year PMBS}

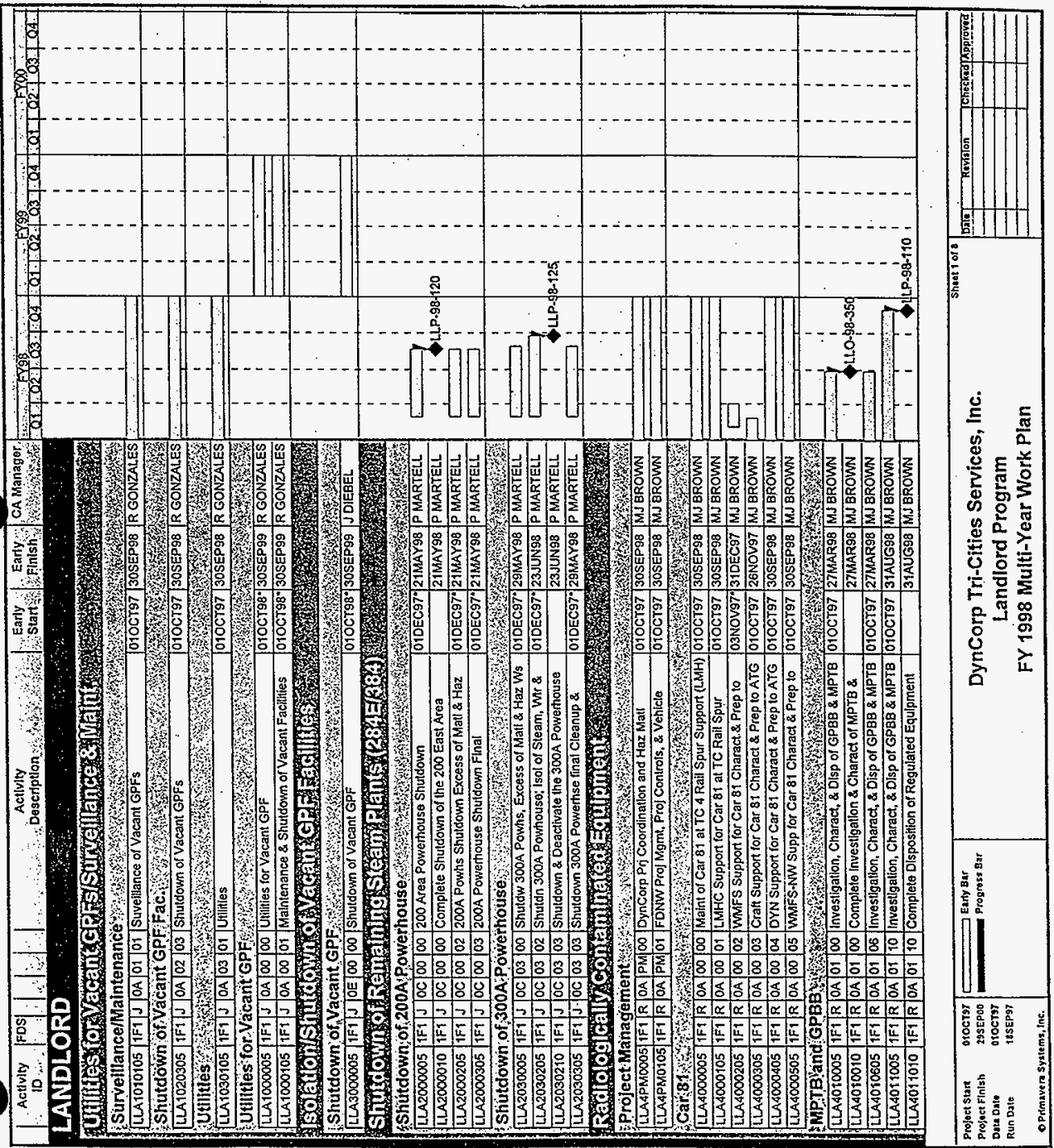




\section{B.3.1/B.3.2 PMBS by PBS/Execution Year PMBS}

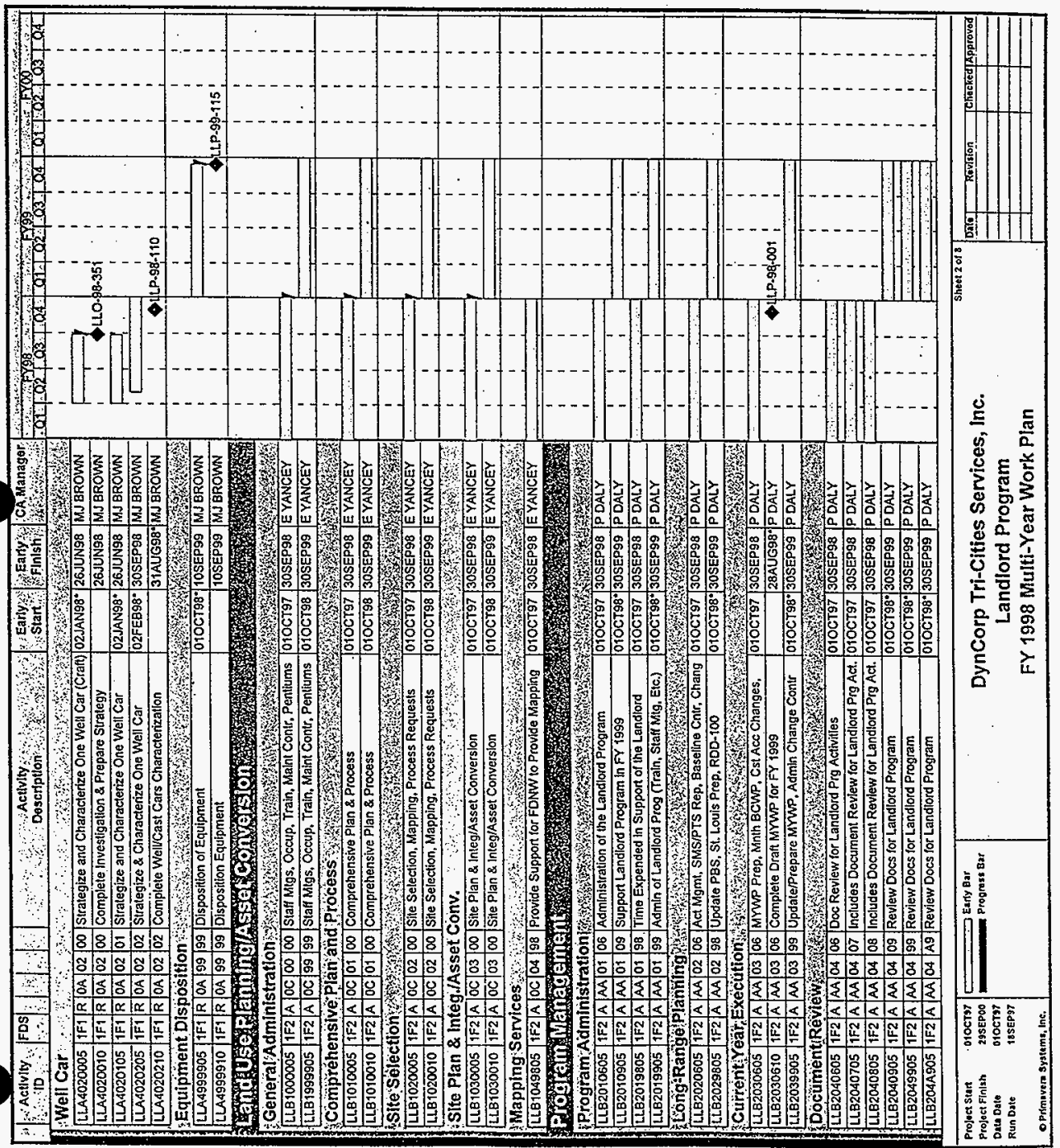




\section{B.3.1/3.3.2 PMBS by PBS/Execution Year PMBS}

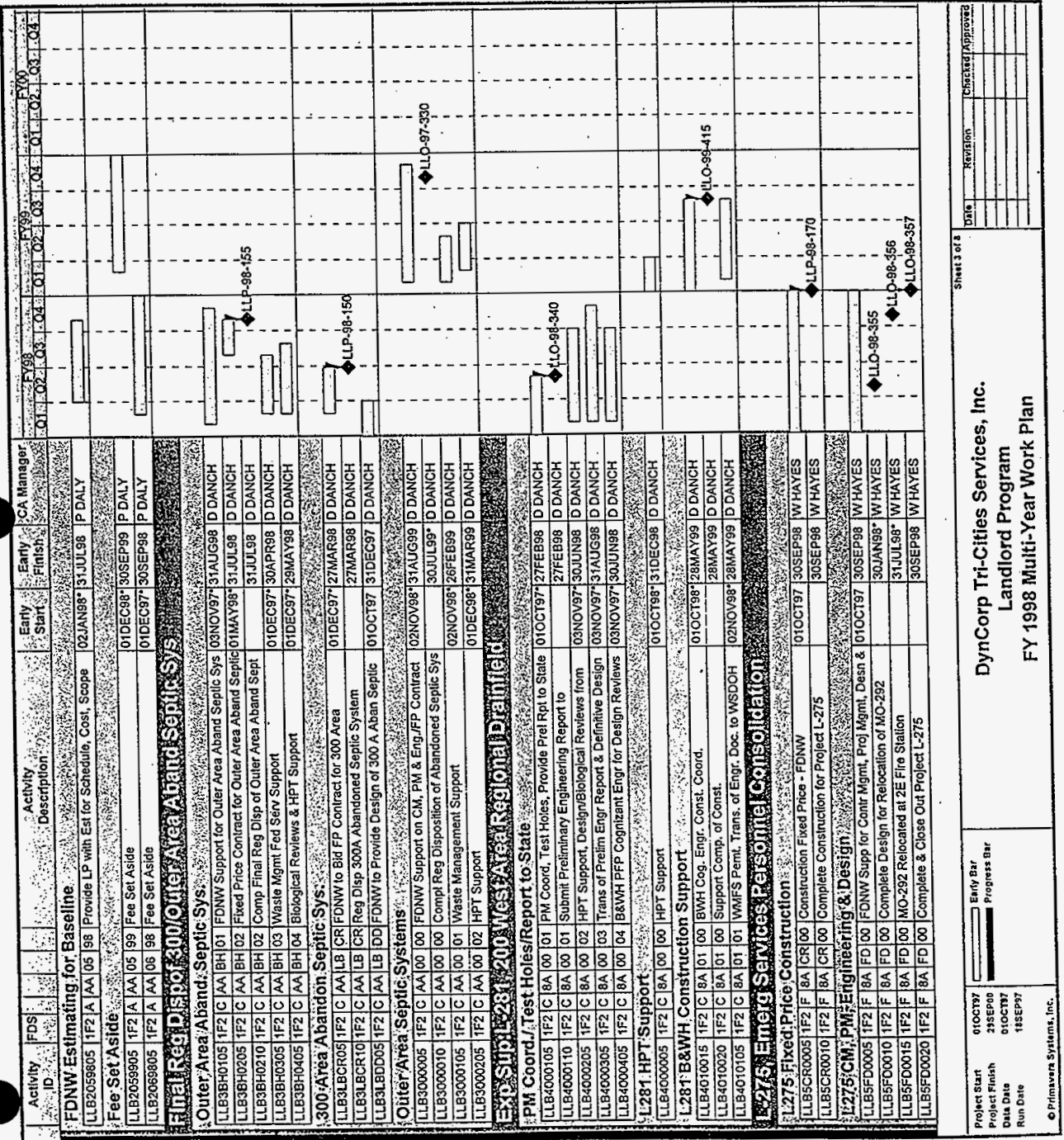




\section{B.3.1/B.3.2 PMBS by PBS/Execution Year PMBS}

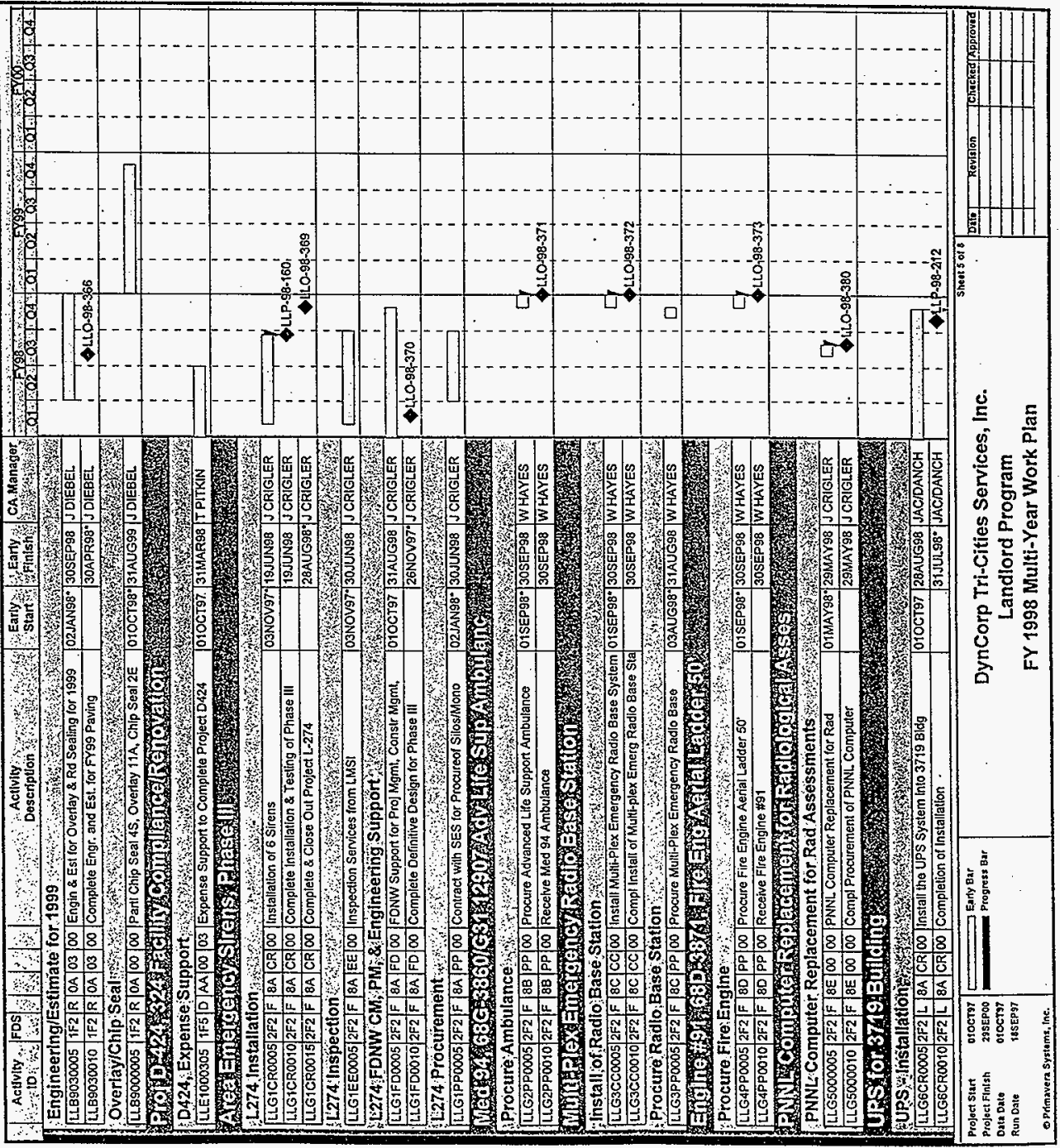




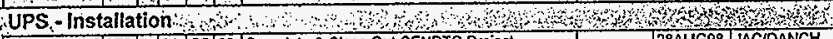

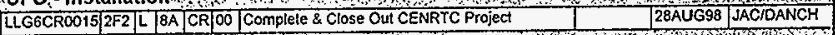

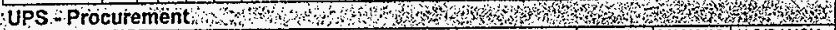

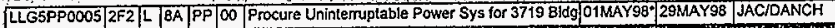
E2813010

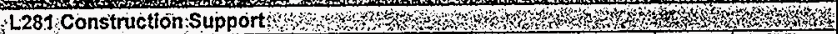

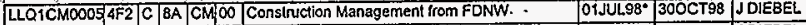

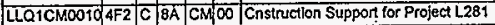
O2NOV98 28 MAY99 J JEIEBEL

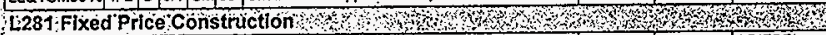

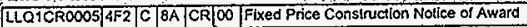
LLOICR0010 4 FF 2 C $\overline{8 \mathrm{~A}}$ CR 00 Construction

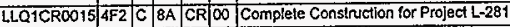

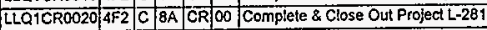
LLQQACR003? 4F2 C BA CR Do IFP Contract 13 NOV98. 13NOV98 $28 \mathrm{MAY} 99$ JDIEBEL

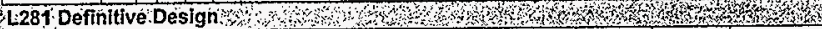

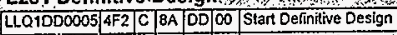

LLQ1DD0010 4F2 C 8 AA DD 00 Definitlve Design Sevices from FDNW

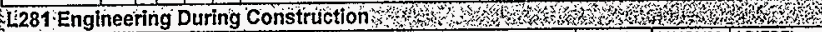

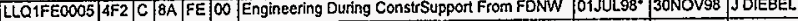

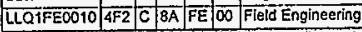
O2NOV $98^{\circ}$ 28MAY99 J DIEBEL.

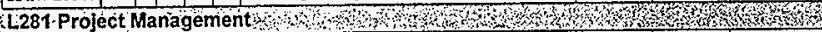

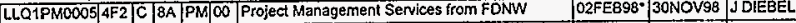

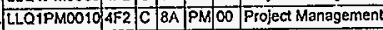

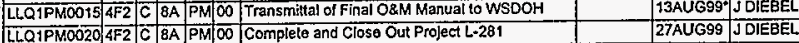

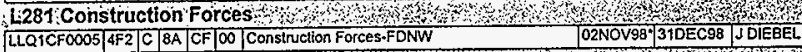

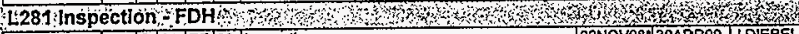

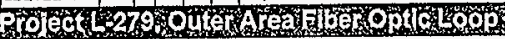

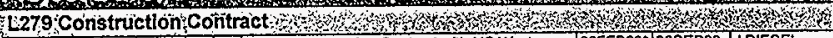

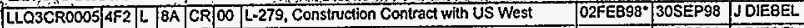
UL3CR0010 4F2 L QA CR के Complete Construction for Project L-279 \begin{tabular}{|l|l|l|l|l|l|l}
\hline$L L Q 3 C R 0105$ & $4 \mathrm{F2}$ & $\mathrm{L}$ & $8 \mathrm{~A}$ & $\mathrm{CR}$ & 01 & Hazardous Material Burtal Costs \\
\hline
\end{tabular} 1279 Defrittivo:Design

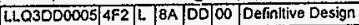

\begin{tabular}{|l|l|l|l|l|l|l}
\hline$L L Q 30 D 0010$ & $4 F 2$ & $L$ & 8A & DO & 00 & Complete Definitive Design For Project L.279 \\
\hline
\end{tabular}

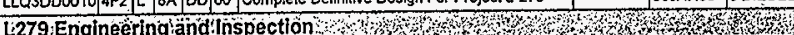

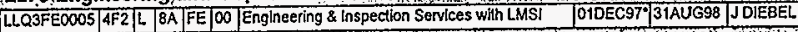

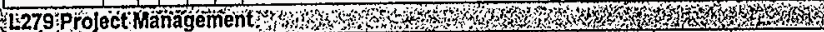

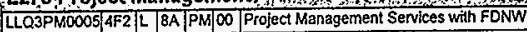

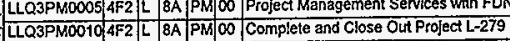
D10CT97 30 SEP98 J DIEBEL \begin{tabular}{|l|l|l|l|l}
\hline $01 . J U L 98^{\circ}$ & $310 E C 98$ & JOLEBEL \\
\hline
\end{tabular} Dens

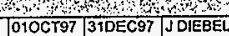
310EC98 JDIEBEL

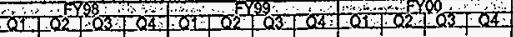
: $:>10-98-360$ :
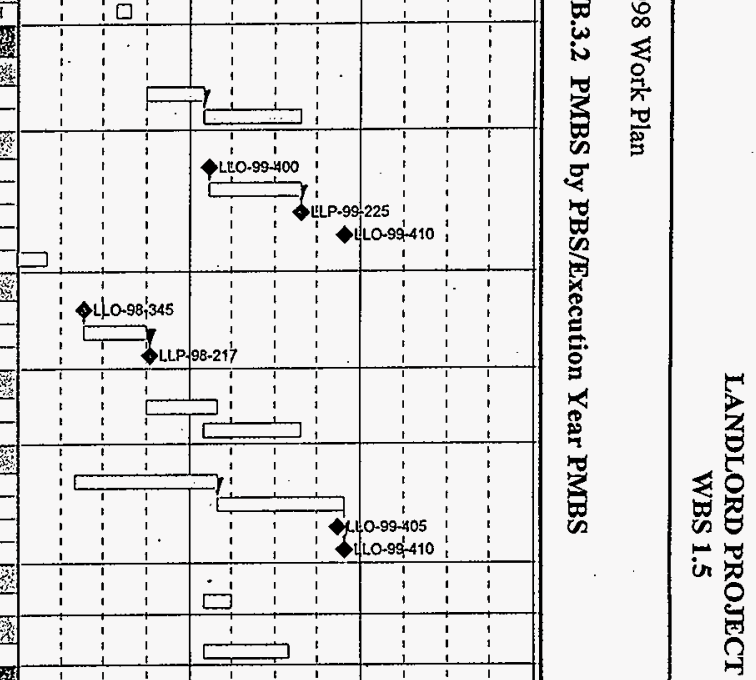

$\begin{array}{ll}\text { Projoct Stant } & 010 \mathrm{CTM7} \\ \text { Projtet Finish } & 295 \mathrm{EP00}\end{array}$

Date Date OTOCT97

OFrimavern Systoms, Ine.
DynCorp Tri-Cities Services, Inc. Landiord Program FY 1998 Multi-Year Work Plan 
B.3.1/B.3.2 PMBS by PBS/Execution Year PMBS

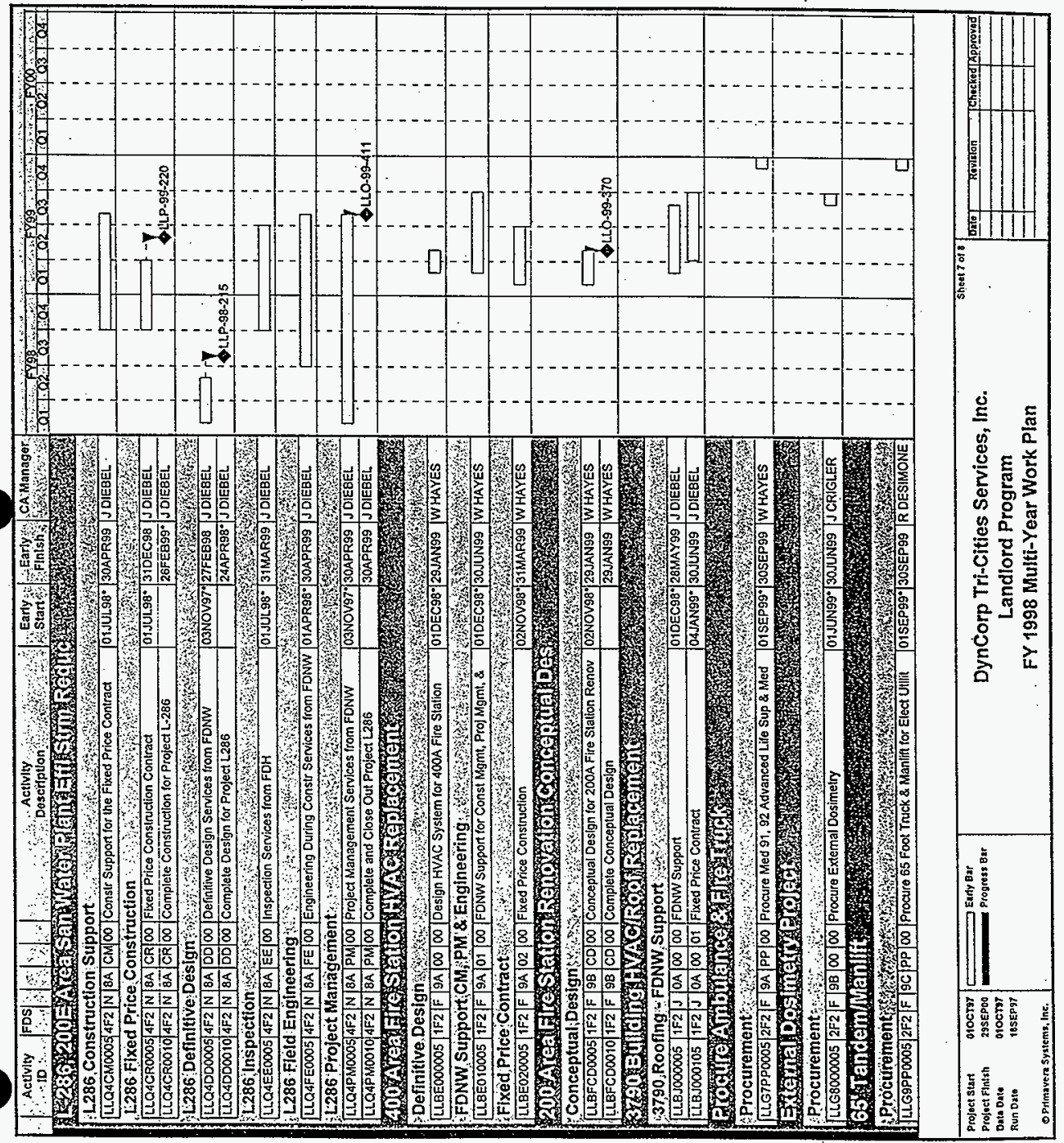




\section{LANDLORD PROJECT \\ WBS 1.5}

FY 1998 Work Plan

HNF-SP-1242, Rev. 0

B.3.1/B.3.2 PMBS by PBS/Execution Year PMBS

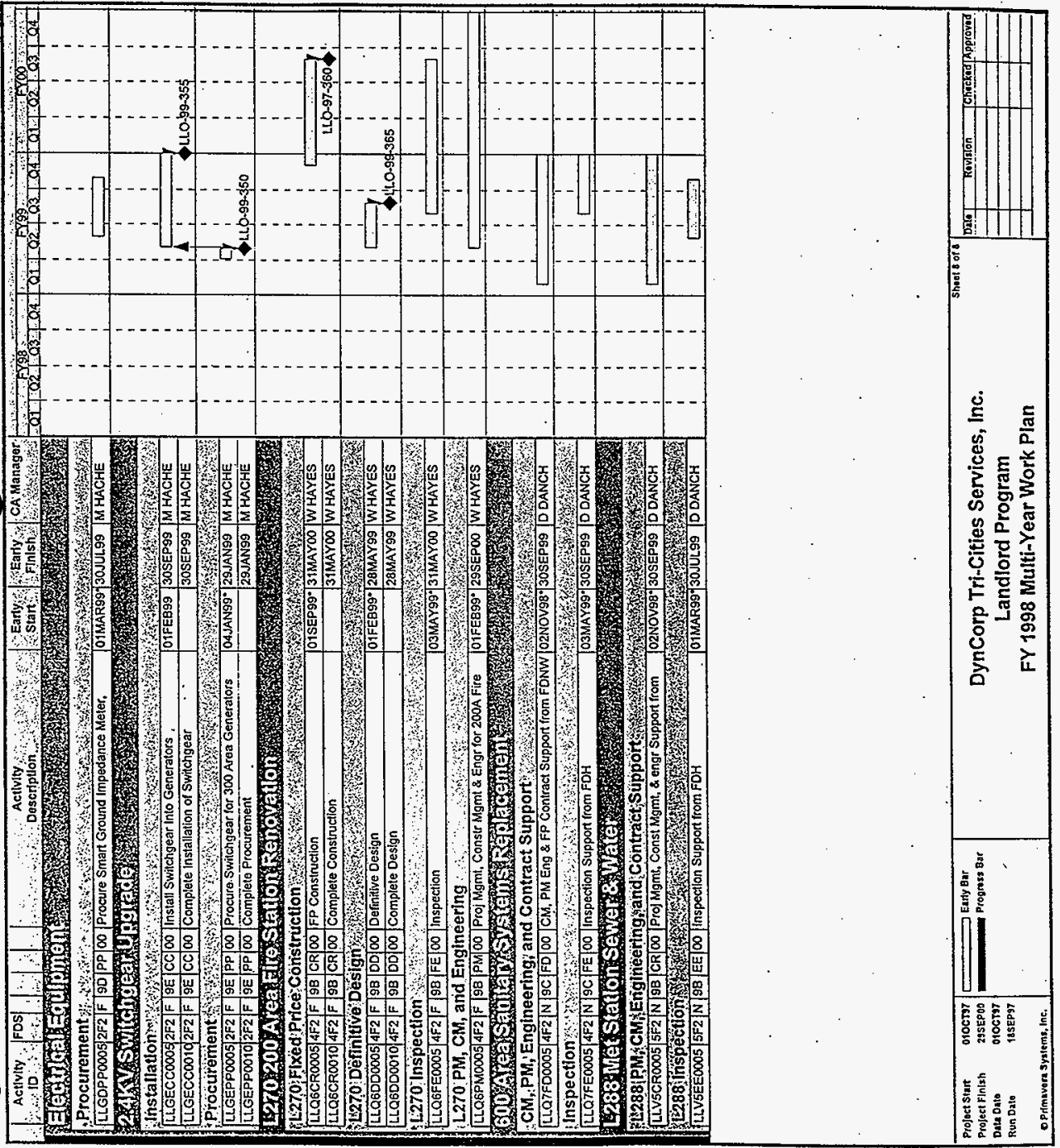




\section{B.3.3 Milestone List (RL, DOE-HQ, EA)}

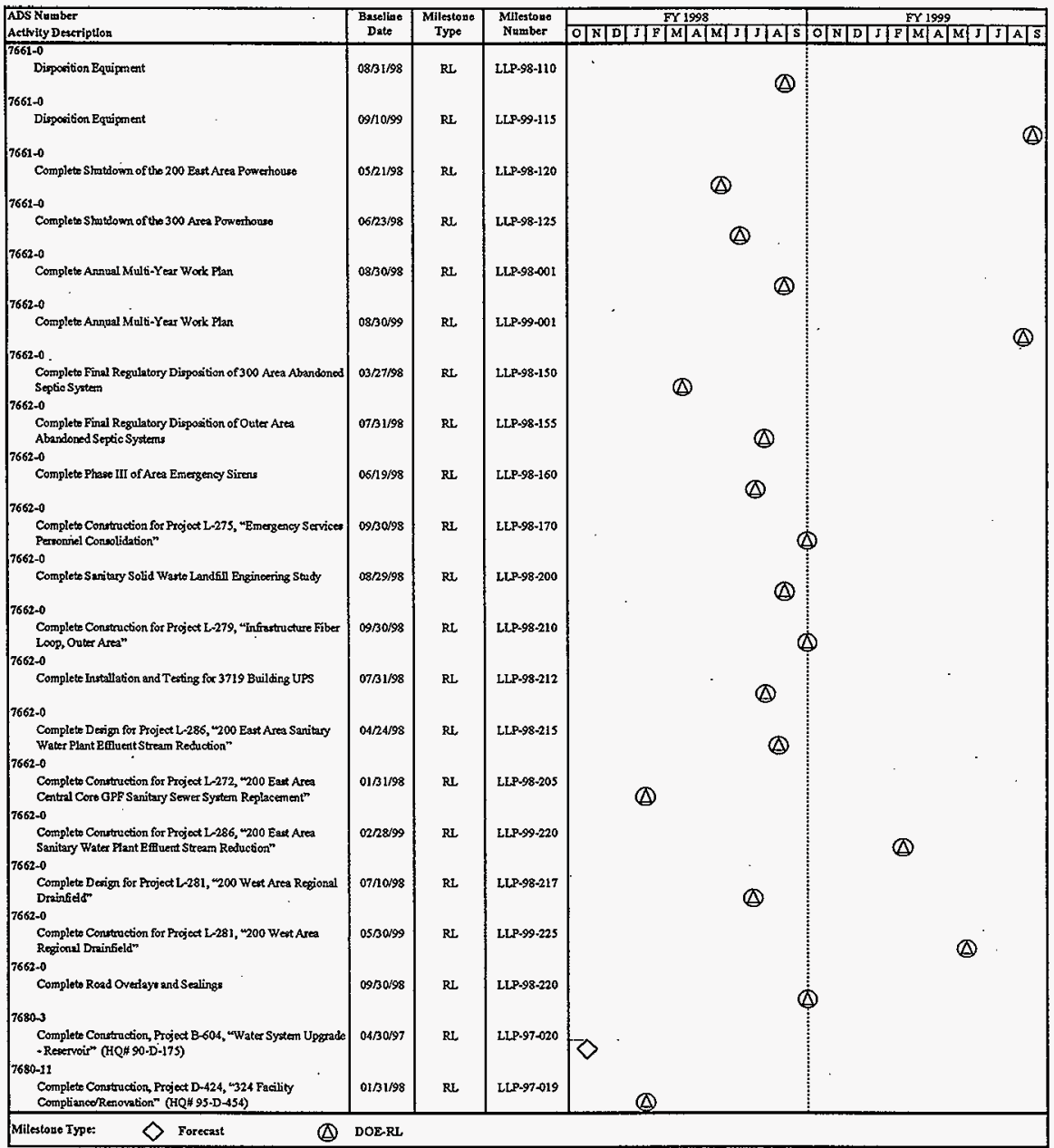




\section{LANDLORD PROJECT}

WBS 1.5

FY 1998 Work Plan

\section{B.3.3 Milestone List (RL, DOE-HQ, EA)}

\begin{tabular}{|c|c|c|c|c|c|}
\hline $\begin{array}{c}\text { Milestone } \\
\text { (M/S) } \\
\text { Type }\end{array}$ & $\begin{array}{c}\text { M/S } \\
\text { Control } \\
\text { Number }\end{array}$ & $\begin{array}{l}\text { WBS } \\
\text { Number }\end{array}$ & $\begin{array}{l}\text { ADS } \\
\text { Number }\end{array}$ & Milestone Description & $\begin{array}{l}\text { Due } \\
\text { Date }\end{array}$ \\
\hline RL & LLP-99-115 & 1.5.1.1.1.4 & $7661-0$ & Disposition Equipment & $09 / 10 / 99$ \\
\hline RL & LLP-98-001 & 1.5.1.1.2.1 & $7662-0$ & Complete Annual Multi-Year Work Plan & $08 / 30 / 98$ \\
\hline$R L$ & LLP-99-001 & 1.5.1.1.2.1 & $7662+0$ & Complete Annual Mutti-Year Work Plan & $08 / 30 / 99$ \\
\hline RL & LLP-98-150 & 1.5.1.1.2.3 & $7662-0$ & $\begin{array}{l}\text { Complete Final Regulatory Disposition of } 300 \text { Area Abandoned Septic } \\
\text { System }\end{array}$ & $03 / 27 / 98$ \\
\hline$R L$ & LLP-98-160 & 1.5.1.1.2.6 & $7662-0$ & Complete Phase III of Area Emergency Sirens & $06 / 19 / 98$ \\
\hline RL & LLP-98-170 & 1.5.1.1.2.6 & $7662-0$ & $\begin{array}{l}\text { Complete Construction for Project L-275, "Emergency Services } \\
\text { Personnel Consolidation" }\end{array}$ & $09 / 30 / 98$ \\
\hline RL & LLP-98-200 & 1.5.1.1.2.7 & $7662-0$ & Complete Sanitary Solid Waste Landfill Engineering Study & $08 / 29 / 98$ \\
\hline RL. & LLP-98-210 & 1.5.1.1.2.8 & $7662-0$ & $\begin{array}{l}\text { Complete Construction for Project L-279, "Infrastructure Fiber Loop, } \\
\text { Outer Area" }\end{array}$ & $09 / 30 / 98$ \\
\hline $\mathrm{RL}$ & LLP-98-212 & 1.5.1.1.2.8 & $7662+0$ & Complete Installation and Testing for 3719 Building UPS & $07 / 31 / 98$ \\
\hline RL & LLP-98-215 & 1.5.1.1.2.9 & $7662-0$ & $\begin{array}{l}\text { Complete Design for Project L-286, "200 East Area Sanitary Water } \\
\text { Plant Effluent Stream Reduction" }\end{array}$ & $04 / 24 / 98$ \\
\hline $\mathrm{RL}$ & LLP-99-225 & 1.5.1.1.2.9 & $7662-0$ & $\begin{array}{l}\text { Complete Construction for Project L-281, " } 200 \text { West Area Regional } \\
\text { Drainfteld" }\end{array}$ & $05 / 30 / 99$ \\
\hline RL & LLبP-98-220 & 1.5 .1 .1 .2 .4 & $7662-0$ & Complete Road Overlays and Sealings & $09 / 30 / 98$ \\
\hline RL & LLP-97-020 & 1.5.1.1.2.9 & $7680+3$ & $\begin{array}{l}\text { Complete Construction, Project B-604, "Water System Upgrade - } \\
\text { Reservoir" (HQ\# 90-D-175) }\end{array}$ & $04 / 30 / 97$ \\
\hline $\mathrm{RL}$ & LLP-97-019 & 1.5 .1 .1 .2 .7 & $7680-11$ & $\begin{array}{l}\text { Complete Construction, Project D-424, "324 Facility } \\
\text { Compliance/Renovation" (HQ\# 95-D-454) }\end{array}$ & $01 / 31 / 98$ \\
\hline
\end{tabular}




\section{B.3.4 Milestone Description Sheets}

\section{PHMC}

MILESTONE DESCRIPTION SHEET

\begin{tabular}{|c|c|c|c|c|}
\hline \multicolumn{4}{|c|}{ Title: DISPOSITION EQUIPMENT } & Date: $8 / 14 / 97$ \\
\hline \multicolumn{4}{|c|}{ Assigned To: LANDLORD PROJECT-DYNCORP } & CIN: \\
\hline \multicolumn{4}{|c|}{ Program UBS Designator: 1.5 .1 .1} & Due Date: $8 / 31 / 98$ \\
\hline \multicolumn{5}{|c|}{ PBS No: RL-TP13 } \\
\hline \multicolumn{2}{|c|}{ MC \#: LLP-98-110 } & TPA NO: & & Rev: 0 \\
\hline $\begin{array}{l}\text { MILESTONE } \\
\text { LEVEL: } \\
\\
\text { DOE-HQ } \\
\times \text { DOE-RL } \\
\text { DOE-FO } \\
\text { CONTRACTOR }\end{array}$ & $\begin{array}{l}\text { HILESTONE } \\
\text { TYPE: } \\
\text { EA } \\
\text { PEG } \\
\text { OTHER }\end{array}$ & $\begin{array}{l}\text { DNFSB STATUS: } \\
\text { DNFSB }(Y / N): N \\
\text { COMMIT } \frac{\hbar}{\hbar}: \\
\text { RECOMM } \frac{H}{\#:}\end{array}$ & $\begin{array}{l}\text { DELIVERABLE: } \\
\text { Report } \\
\text { letter } \\
\text { Drawing(s) } \\
\text { X other (Specify) } \\
\text { ACCEPTANCE OF } \\
\text { COMPLETEO WORK }\end{array}$ & $\begin{array}{l}\text { ADDRESS TO: } \\
\text { DOE-HQ } \\
\times \text { DOE-RL } \\
X \text { Other (Specify) } \\
\text { FDH DIRECTOR OF } \\
\text { INF. \& SEC. }\end{array}$ \\
\hline \multicolumn{5}{|c|}{$\begin{array}{l}\text { Milestone Description: } \\
\text { Complete disposition of regulated equipment as follows: } \\
\text { Characterize and disposition Nulti-Purpose Transfer Box (MPTB) HO-1OA- } \\
\text { 19370; Characterize and disposition General Purpose Burial Box (GPBB) HO- } \\
\text { 10A-19308; Complete Well/Cask Cars Characterization Strategy. } \\
\quad:\end{array}$} \\
\hline \multicolumn{5}{|c|}{$\begin{array}{l}\text { Description of what constitutes completion of this milestone: } \\
\text { The GPBB and MPTB dispositioning will be complete upon obtaining signatures } \\
\text { on the Acceptance of Completed Work, which will include copies of the } \\
\text { signed acceptance from the Low Level Waste Burial Ground signifying burial } \\
\text { of the contaminated equipment. } \\
\text { The Weil/Cask Cars Characterization Strategy will be complete when the plan . } \\
\text { is approved by the following: } \\
\text { DOE-RL: Representative from the Site Infrastructure Division } \\
\text { FDH: : Director of Infrastructure and Security or Designee } \\
\text { DynCorp: Landlord Project Technical Representative } \\
\text { DynCorp: Environmental Science Technical Representative } \\
\end{array}$} \\
\hline
\end{tabular}




\section{B.3.4 Milestone Description Sheets}

\section{PHMC}

MILESTONE DESCRIPTION SHEET

\begin{tabular}{|c|c|c|c|c|}
\hline \multicolumn{4}{|c|}{ TitTe: DISPOSITION EQUIPMENT } & Date: $8 / 14 / 97$ \\
\hline \multicolumn{4}{|c|}{ Assigned TO: LANDLORD PROJECT-DYNCORP } & CIN: \\
\hline \multicolumn{4}{|c|}{ Program WBS Designator: 1.5.1.1 } & Due Date: $9 / 10 / 99$ \\
\hline \multicolumn{5}{|c|}{ PBS No: RL-TP13 } \\
\hline \multicolumn{2}{|c|}{ MC \#: LLP-99-115 } & TPA No: & & Rev: 0 \\
\hline $\begin{array}{l}\text { MILESTONE } \\
\text { LEVEL: } \\
\text { DOE-HQ } \\
\text { DOE-HQ } \\
\text { DOE-RL } \\
\text { DOE-FO } \\
\text { CONTRACTOR }\end{array}$ & $\begin{array}{c}\text { MILESTONE } \\
\text { TYPE: } \\
\text { EA } \\
\text { PEG } \\
\text { OTHER }\end{array}$ & $\begin{array}{l}\text { DNFSB STATUS: } \\
\text { DNFSB (Y/N): } N \\
\text { COMMIT. H: } \\
\text { RECOMH H: } \\
\end{array}$ & $\begin{array}{l}\text { DELIVERABLE: } \\
\text { Report } \\
\text { Letter } \\
\text { Drawing(s) } \\
\text { X Other (Specify) } \\
\text { ACCEPTANCE OF } \\
\text { COMPLETED WORK. }\end{array}$ & $\begin{array}{l}\text { ADDRESS TO: } \\
\text { DOE-HQ } \\
\times \text { DOE-RL } \\
X \text { Other (Specify) } \\
\text { FDH DIRECTOR OF } \\
\text { INF. \& SEC. }\end{array}$ \\
\hline \multicolumn{5}{|c|}{$\begin{array}{l}\text { Milestone Description: } \\
\text { Complete disposition of one Weli/Cask Car. }\end{array}$} \\
\hline \multicolumn{5}{|c|}{$\begin{array}{l}\text { Description of what constitutes completion of this milestone: } \\
\text { Signed Acceptance of Completed Work (ACW), which includes signed acceptance } \\
\text { from the Low Level Haste Burial Grounds signifying burial of contaminated } \\
\text { elements of the Well/Cask Car. } \\
\text { The ACW is to be signed by the following: } \\
\text { DOE-RL: Representative of Site Infrastructure Division } \\
\text { FDH: } \quad \text { Director of Infrastructure and Security or Designee } \\
\text { DynCorp: Landlord Project Technical Representative } \\
\text { DynCorp: Environmental Science Technical Representative }\end{array}$} \\
\hline
\end{tabular}




\section{LANDLORD PROJECT \\ WBS 1.5}

FY 1998 Work Plan

HNF-SP-1242, Rev. 0

\section{B.3.4 Milestone Description Sheets}

\section{PHMC}

MILESTONE DESCRIPTION SHEET

\begin{tabular}{|c|c|c|c|c|}
\hline \multicolumn{4}{|c|}{$\begin{array}{l}\text { Title: COMPLETE SHUTDONN OF THE } 200 \text { EAST AREA } \\
\text { POWERHOUSE }\end{array}$} & Date: $8 / 14 / 97$ \\
\hline \multicolumn{4}{|c|}{ Assigned TO: LANOLORD PROJECT-DYNCORP } & CIN: \\
\hline \multicolumn{4}{|c|}{ Program WBS Designator: 1.5.1.I } & Due Date: $5 / 21 / 98$ \\
\hline \multicolumn{5}{|c|}{ PBS No: RL-TP13 } \\
\hline \multicolumn{2}{|c|}{ MC \#: LLP-98-120 } & TPA No: & & Rev: 0 \\
\hline $\begin{array}{l}\text { MILESTONE } \\
\text { LEVEL: } \\
\\
\text { DOE-HQ } \\
\text { X DOE-RL } \\
\text { OOE-FO } \\
\text { CONTRACTOR }\end{array}$ & $\begin{array}{l}\text { MILESTONE } \\
\text { TYPE: } \\
\text { EA } \\
\text { PEG } \\
\text { OTHER }\end{array}$ & $\begin{array}{l}\text { DNFSB STATUS: } \\
\text { DNFSB }(Y / N): N \\
\text { COMHIT } \#: \\
\text { RECONM } \\
\end{array}$ & $\begin{array}{l}\text { DELIVERABLE: } \\
\text { Report } \\
\text { Letter } \\
\text { Drawing(s) } \\
\text { X Other (Specify) } \\
\text { ACCEPTANCE OF } \\
\text { COMPLETEO HORK }\end{array}$ & $\begin{array}{l}\text { ADDRESS TO: } \\
\text { DOE-HQ } \\
X \text { DOE-RL } \\
X \text { Other (Specify) } \\
\text { FOH DIRECTOR OF } \\
\text { INF. \& SEC. }\end{array}$ \\
\hline \multicolumn{5}{|c|}{$\begin{array}{l}\text { Milestone Description: } \\
\text { Shutdown and deactivate the } 284 \text { East Powerhouse within } 120 \text { working days of } \\
\text { receiving written direction from RL-SID to do so. This milestone currently } \\
\text { assumes a starting date of December } 1,1997 .\end{array}$} \\
\hline \multicolumn{5}{|c|}{$\begin{array}{l}\text { Description of what constitutes completion of this milestone: } \\
\text { Acceptance of Completed Work signed by the following: } \\
\text { DOE-RL: Representative fron the Site Infrastructure Division } \\
\text { FDH: } \\
\text { DynCorp: Manager, Utilities } \\
\text { DynCorp: Landiord Project Technical Representative } \\
\end{array}$} \\
\hline
\end{tabular}




\section{B.3.4 Milestone Description Sheets}

\section{PHMC}

MILESTONE DESCRIPTION SHEET

\begin{tabular}{|c|c|c|c|c|}
\hline \multicolumn{4}{|c|}{ Title: COMPLETE ANNUAL MULTI-YEAR HORK PLAN } & Date: $8 / 11 / 97$ \\
\hline \multicolumn{4}{|c|}{ Assigned To: LANDLORD PROJECT-DYNCORP } & CIN: \\
\hline \multicolumn{4}{|c|}{ Program WBS Designator: 1.5 .1 .1} & Due Date: $8 / 30 / 98$ \\
\hline \multicolumn{5}{|c|}{ PBS No: RL-TP13 } \\
\hline \multicolumn{2}{|c|}{ KC \#: LLP-98-001 } & TPA No: & & Rev: 0 \\
\hline $\begin{array}{l}\text { MILESTONE. } \\
\text { LEVEL: } \\
\\
\text { DOE-HQ } \\
\text { DOEE-RL } \\
\text { DOE-FO } \\
\text { CONTRACTOR }\end{array}$ & $\begin{array}{l}\text { MILESTONE } \\
\text { TYPE: } \\
\text { EA } \\
\text { PEG } \\
\text { OTHER }\end{array}$ & $\begin{array}{l}\text { DNFSB STATUS: } \\
\text { DNFSB }(Y / N): N \\
\text { COMMIT } \frac{\pi}{\pi}: \\
\text { RECOMM } \#:\end{array}$ & $\begin{array}{l}\text { DELIVERABLE: } \\
\text {. } \\
\text { Report } \\
\text { Letter } \\
\text { Drawing(s) } \\
\text { X Other (Specify) } \\
\text { MULTI-YEAR WORK } \\
\text { PLAN }\end{array}$ & $\begin{array}{l}\text { ADDRESS TO: } \\
\text { DOE-HQ } \\
\times \text { DOE-RL } \\
x \text { Other (Specify) } \\
\text { FOH DIRECTOR OF } \\
\text { INF. \& SEC. }\end{array}$ \\
\hline \multicolumn{5}{|c|}{$\begin{array}{l}\text { Milestone Description: } \\
\text { The Multi-Year Work Plan is the program baseline document for Fiscal Year } \\
1999 \text {. }\end{array}$} \\
\hline \multicolumn{5}{|c|}{$\begin{array}{l}\text { Description of what constitutes completion of this milestone: } \\
\text { Transmit Multi-Year Work Plan by letter to the director of FDH } \\
\text { Infrastructure and Security and RL's Site Infrastructure Division by August } \\
30,1998 \text {. }\end{array}$} \\
\hline
\end{tabular}




\section{B.3.4 Milestone Description Sheets}

\section{PHMC}

MILESTONE DESCRIPTION SHEET

\begin{tabular}{|c|c|c|c|c|}
\hline \multicolumn{4}{|c|}{ Title: COMPLETE ANNUAL MULTI-YEAR WORK PLAN } & te: $8 / 20 / 97$ \\
\hline \multicolumn{4}{|c|}{ Assigned To: LANDLORD PROJECT-DYNCORP } & CIN: \\
\hline \multicolumn{4}{|c|}{ Program WBS Designator: 1.5 .1 .1} & Due Date: $8 / 30 / 99$ \\
\hline \multicolumn{5}{|c|}{ PBS No: RL-TP13 - } \\
\hline \multicolumn{2}{|c|}{ HC \#: :LLP -99-001 } & \multicolumn{2}{|l|}{ TPA No: } & Rev: 0 \\
\hline $\begin{array}{l}\text { MILESTONE } \\
\text { LEVEL: } \\
\text { DOE-HQ } \\
\times \text { DOE-RL } \\
\text { DOE-FO } \\
\text { CONTRACTOR }\end{array}$ & $\begin{array}{l}\text { MILESTONE } \\
\text { TYPE: } \\
\text { EA } \\
\text { PEG } \\
\text { OTHER }\end{array}$ & $\begin{array}{l}\text { DNFSB STATUS: } \\
\text { DNFSB }(\dot{Y} / N): N \\
\text { COMMIT } \#: \\
\text { RECOMM } \frac{\pi}{\pi}:\end{array}$ & $\begin{array}{l}\text { DELIVERABLE: } \\
\\
\text { Report } \\
\text { Letter } \\
\text { Drawing (s) } \\
\text { X Other (Specify) } \\
\text { MULYI-YEAR WORK } \\
\text { PLAN }\end{array}$ & $\begin{array}{l}\text { ADDRESS TO: } \\
\text { DOE-HQ } \\
\times \text { DOE-RL } \\
\times \text { Dther (Specify) } \\
\text { FDH DIRECTOR OF } \\
\text { INF. \& SEC. }\end{array}$ \\
\hline \multicolumn{5}{|c|}{$\begin{array}{l}\text { Milestone Description: } \\
\text { The Multi-Year Work Plan is the program baseline document for Fiscal Year } \\
2000 \text {. }\end{array}$} \\
\hline \multicolumn{5}{|c|}{$\begin{array}{l}\text { Description of what constitutes completion of this milestone: } \\
\text { Transmit Multi-Year Hork PTan by letter to the Director of FDH } \\
\text { Infrastructure and Security and RL's Site Infrastructure Division by August } \\
30,1999 \text {. Obtain FDH and RL approval for work authorization prior to the } \\
\text { beginning of FY } 2000 \text {. }\end{array}$} \\
\hline
\end{tabular}




\section{LANDLORD PROJECT \\ WBS 1.5}

FY 1998 Work Plan

HNF-SP-1242, Rev. 0

B.3.4 Milestone Description Sheets

PHMC

MILESTONE DESCRIPTION SHEET

\begin{tabular}{|c|c|c|c|c|}
\hline \multicolumn{4}{|c|}{$\begin{array}{l}\text { Title: COMPLETE FINAL REG. DISPOSITION OF } 300 \text { AREA } \\
\text { ABANDONEO SEPTIC SYS. }\end{array}$} & Date: $8 / 14 / 97$ \\
\hline \multicolumn{4}{|c|}{ Assigned To: LANOLORO PROJECT-DYNCORP } & CIN: \\
\hline \multicolumn{4}{|c|}{ Program KBS Designator: 1.5 .1 .1} & Due Date: $3 / 27 / 98$ \\
\hline \multicolumn{5}{|c|}{ PBS No: RL-TP13 } \\
\hline \multicolumn{2}{|c|}{ MC \#: LLP-98-150 } & TPA NO: & & Rev: 0 \\
\hline $\begin{array}{l}\text { MILESTONE } \\
\text { LEVEL: } \\
\text { DOE-HQ } \\
\times \text { DOE-RL } \\
\text { DOE-FO } \\
\text { CONTRACTOR } \\
.\end{array}$ & $\begin{array}{l}\text { MILESTONE } \\
\text { TYPE: } \\
\text { EA } \\
\text { PEG } \\
\text { OTHER } \\
\end{array}$ & $\begin{array}{l}\text { DNFSB STATUS: } \\
\text { DNFSB }(Y / N): N \\
\text { COMMIT } \frac{H}{\pi}: \\
\text { RECONH } \frac{H}{\pi}:\end{array}$ & $\begin{array}{l}\text { DELIVERABLE: } \\
\text { Report } \\
\text { Letter } \\
\text { Drawing(s) } \\
\text { X Other (Specify) } \\
\text { ACCEPTANCE OF } \\
\text { COMPLETED WORK }\end{array}$ & $\begin{array}{l}\text { ADDRESS TO: } \\
\text { DOE-HQ } \\
X \text { DOE-RL } \\
X \text { Other (Specify) } \\
\text { FOH DIRECTOR OF } \\
\text { INF. \& SEC. }\end{array}$ \\
\hline \multicolumn{5}{|c|}{$\begin{array}{l}\text { Milestone Description: } \\
\text { Regulatorially disposition the Abandoned } 300 \text { Area Septic System to meet } \\
\text { Washington Administrative Codes. This work jncludes complete isolation of } \\
\text { the septic tank, destruction of all pathogens, and filling the tank with } \\
\text { soil. The septic tank waste water trench will be abandoned in place and } \\
\text { requires no further action. }\end{array}$} \\
\hline \multicolumn{5}{|c|}{$\begin{array}{l}\text { Description of what constitutes completion of this milestone: } \\
\text { Acceptance of Completed Work signed by the following: } \\
\text { DOE-RL: Representative from the Site Infrastructure Division } \\
\text { FOH: } \quad \text { Director of Infrastructure and Security or Designee } \\
\text { DynCorp: Manager, } 300 \text { Area Sanitary Systems } \\
\text { DynCorp: Landlord Project Technical Representative } \\
\end{array}$} \\
\hline
\end{tabular}




\section{LANDLORD PROJECT \\ WBS 1.5}

FY 1998 Work Plan

HNF-SP-1242, Rev. 0

B.3.4 Milestone Description Sheets

PHMC

MILESTONE DESCRIPTION SHEET

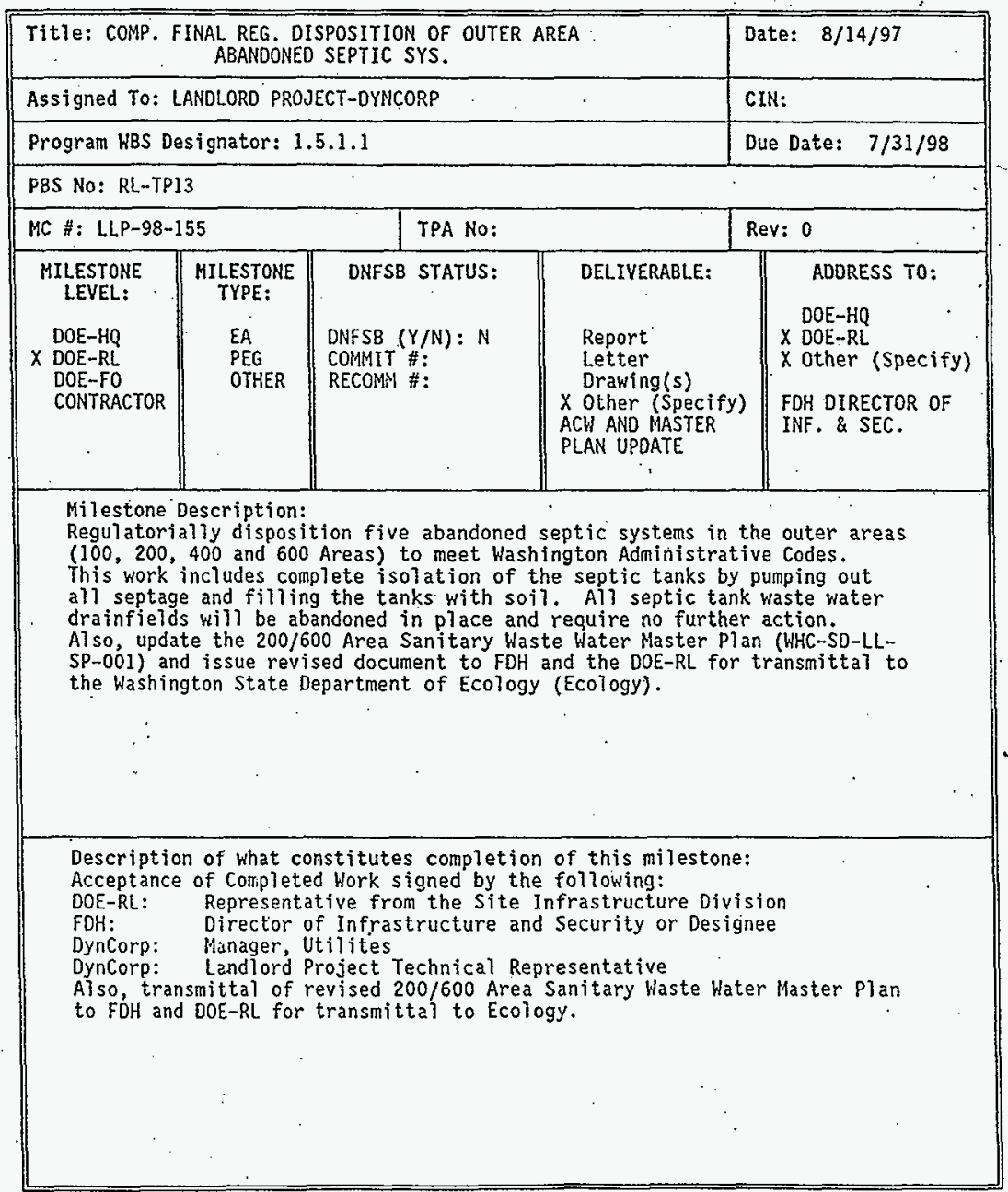




\section{LANDLORD PROJECT}

WBS 1.5

FY 1998 Work Plan

HNF-SP-1242, Rev. 0

\section{B.3.4 Milestone Description Sheets}

\section{PHMC}

MILESTONE DESCRIPTION SHEET

\begin{tabular}{|c|c|c|c|c|}
\hline \multicolumn{4}{|c|}{ Tit7e: COMPLETE PHASE III OF AREA EMERGENCY SIRENS } & Date: $8 / 20 / 97$ \\
\hline \multicolumn{4}{|c|}{ Assigned TO: LANDLORD PROJECT-DYNCORP } & CIN: \\
\hline \multicolumn{4}{|c|}{ Program HBS Designator: 1.5 .1 .1} & Due Date: $6 / 19 / 98$ \\
\hline \multicolumn{5}{|c|}{ PBS No: RL-TP13 } \\
\hline \multicolumn{2}{|c|}{ MC \# : L.LP-98-160 } & TPA No: & & Rev: 0 . \\
\hline $\begin{array}{l}\text { MILESTONE } \\
\text { LEVEL: } \\
\\
\text { DOE-HQ } \\
\times \text { DOE-RL } \\
\text { DOE-FO } \\
\text { CONTRACTOR }\end{array}$ & $\begin{array}{l}\text { MYLESTONE } \\
\text { TYPE: } \\
\text { EA } \\
\text { PEG } \\
\text { OTHER }\end{array}$ & $\begin{array}{l}\text { DNFSB STATUS: } \\
\text { DNFSB }(Y / N): N \\
\text { COMHIT } \frac{N}{11}: \\
\text { RECOMM } \frac{ \pm}{\pi}:\end{array}$ & $\begin{array}{l}\text { DELIVERABLE: } \\
\\
\text { Report } \\
\text { Letter } \\
\text { Drawing(s) } \\
\text { X Other (Specify) } \\
\text { ACCEPTANCE OO } \\
\text { COMPLETEO WORK }\end{array}$ & $\begin{array}{l}\text { ADDRESS TO: } \\
\text { DOE-HQ } \\
\times \text { DOE-RL } \\
\times \text { Other (Specify) } \\
\text { FOH DIRECTOR OF } \\
\text { INF. \& SEC. }\end{array}$ \\
\hline \multicolumn{5}{|c|}{$\begin{array}{l}\text { Milestone Description: } \\
\text { Complete installation and testing of the Phase In Area Emergency Sirens } \\
\text { along the Columbia River and in the } 400 \text { Area. }\end{array}$} \\
\hline \multicolumn{5}{|c|}{$\begin{array}{l}\text { Description of what constitutes completion of this milestone: } \\
\text { Acceptance of Completed Work signed by the following: } \\
\text { DOE-RL: Representative for the Site Infrastructure Division } \\
\text { FDH: } \quad \text { Director of Infrastructure and Security or Designee } \\
\text { DynCorp: Manager of Emergency Preparedness } \\
\text { DynCorp: Landlord Project Technical Representative } \\
\end{array}$} \\
\hline
\end{tabular}




\section{LANDLORD PROJECT \\ WBS 1.5}

FY 1998 Work Plan

HNF-SP-1242, Rev. 0

B.3.4 Milestone Description Sheets

PHMC
MILESTONE DESCRIPTION SHEET

\begin{tabular}{|c|c|c|c|c|}
\hline \multicolumn{4}{|c|}{ Title: COMPLETE CONSTRUCTION FOR PROJECT L-275 } & Date: $8 / 20 / 97$ \\
\hline \multicolumn{4}{|c|}{ Assigned TO: LANDLORD PROJECT-DYNCORP } & CIN: \\
\hline \multicolumn{4}{|c|}{ Program HBS Designator: 1.5 .1 .1} & Due Date: $9 / 30 / 98$ \\
\hline \multicolumn{5}{|c|}{ PBS No: RL-TP13 } \\
\hline \multicolumn{2}{|c|}{ MC \#: LLP-98-170 } & TPA No: & & Rev: 0 \\
\hline $\begin{array}{l}\text { MILESTONE } \\
\text { LEVEL: }\end{array}$ & $\begin{array}{l}\text { MILESTONE } \\
\text { TYPE: }\end{array}$ & DNFSB STATUS: & DELIVERABLE: & \multirow{2}{*}{$\begin{array}{l}\text { ADDRESS TO: } \\
\text { DOE-HQ } \\
\times \text { DOE-RL } \\
\times \text { Other (SPecify) } \\
\text { FDH DIRECTOR OF } \\
\text { INF. \& SEC. }\end{array}$} \\
\hline $\begin{array}{l}\text { DOE-HQ } \\
\times \text { DOE-RL } \\
\text { DOE-FO } \\
\text { CONTRACTOR }\end{array}$ & $\begin{array}{l}\text { EA } \\
\text { PEG } \\
\text { OTHER }\end{array}$ & $\begin{array}{l}\text { DNFSB }(Y / N): N \\
\text { COMMIT \#: } \\
\text { RECOMM \#: }\end{array}$ & $\begin{array}{l}\text { Report } \\
\text { Letter } \\
\text { Drawing (s) } \\
\times \text { Other (Specify) } \\
\text { ACCEPTANCE OF } \\
\text { COMPLETED WORK }\end{array}$ & \\
\hline
\end{tabular}

Milestone Description:

Relocate existing modular office complex (M0-292) to the west of the 200

Area Fire Station Complex $(609 \mathrm{~A} / \mathrm{C})$; including ail site preparation work.

Also, convert modular office $609 \mathrm{G}$ into the Respirator Protection

Maintenance and Overhaul Turnaround Shop, which includes relocation of the compressor system currentiy located in 609A.

Description of what constitutes completion of this milestone:

Completion of this project will include M0-292 ready for occupancy by

Emergency Services Personnel, $609 \mathrm{G}$ renovated to accept compressor equipment

located in 609A, and equipment from $609 \mathrm{~A}$ installed and operational in the

renovated 609G.

Two separate Acceptance of Completed Work forms (one for M0-292 Occupancy,

and one for $609 \mathrm{G}$ conversion/operations) signed by the following:

DOE-RL: Representative from Site Infrastructure Division

FDH: $\quad$ Director of Infrastructure and Security or Designee

DynCorp: Chief, Hanford Fire Department or Designee

DynCorp: Landlord Project Technical Representative 


\section{B.3.4 Milestone Description Sheets}

\section{PHMC}

MILESTONE DESCRIPTION SHEET

\begin{tabular}{|c|c|c|c|c|}
\hline \multicolumn{4}{|c|}{$\begin{array}{l}\text { Title: COMPLETE SANITARY SOLID WASTE LANDFILL } \\
\text { ENGINEERING STUDY }\end{array}$} & Date: $8 / 20 / 98$ \\
\hline \multicolumn{4}{|c|}{ Assigned TO: LANOLORO FUNOING } & CIN: \\
\hline \multicolumn{4}{|c|}{ Program WBS Designator: 1.5 .1 .1} & Due Date: $8 / 29 / 98$ \\
\hline \multicolumn{5}{|c|}{ PBS No: RL-TP13 } \\
\hline \multicolumn{2}{|c|}{ MC \#: LLP-98-200 } & TPA No: & & Rey: 0 \\
\hline $\begin{array}{l}\text { MILESTONE } \\
\text { LEVEL: } \\
\text { DOE-HQ } \\
\times \text { DOE-RL } \\
\text { DOE-FO } \\
\text { CONTRACTOR }\end{array}$ & $\begin{array}{l}\text { MILESTONE } \\
\text { TYPE: } \\
\text { EA } \\
\text { PEG } \\
\text { OTHER }\end{array}$ & $\begin{array}{l}\text { DNFSB STATUS: } \\
\text { ONFSB }(Y / N): N \\
\text { COMIIT \#: } \\
\text { RECOMM \#: }\end{array}$ & $\begin{array}{l}\text { DELIVERABLE: } \\
\text { Report } \\
\text { Letter } \\
\text { Drawing ( } s \text { ) } \\
\text { X Other (Specify) } \\
\text { ENGINEERING STUDY }\end{array}$ & $\begin{array}{l}\text { ADDRESS TO: } \\
\text { DOE-HQ } \\
\times \text { DOE-RL } \\
X \text { Other (Specify) } \\
\text { FOH DIRECTOR OF } \\
\text { INF. \& SEC. }\end{array}$ \\
\hline \multicolumn{5}{|c|}{$\begin{array}{l}\text { Milestone Description: } \\
\text { Complete preliminary engineering (evaluation of alternatives) study for the } \\
\text { final closure of the Hanford Sanitary Solid Haste Landfilt. }\end{array}$} \\
\hline \multicolumn{5}{|c|}{$\begin{array}{l}\text { Description of what constitutes completion of this milestone: } \\
\text { Transmittal of final engineering study by letter from the FDH Director of } \\
\text { Infrastructure and Security to the DOE-RL Director of the Site } \\
\text { Infrastructure Division. }\end{array}$} \\
\hline
\end{tabular}




\section{LANDLORD PROJECT. \\ WBS 1.5}

FY 1998 Work Plan

HNF-SP-1242, Rev. 0

\section{B.3.4 Milestone Description Sheets}

\section{PHMC}

MILESTONE DESCRIPTION SHEET

\begin{tabular}{|c|c|c|c|c|}
\hline \multicolumn{4}{|c|}{$\begin{array}{c}\text { Title: CONPLETE INSTALLATION AND TESTING OF } 3719 \\
\text { BUILDING UPS }\end{array}$} & Date: $8 / 14 / 97$ \\
\hline \multicolumn{4}{|c|}{ Assigned TO: LANDLORD PROJECT-DYNCORP } & CIN: \\
\hline \multicolumn{4}{|c|}{ Program HBS Designator: 1.5.1.1 } & Due Date: $7 / 31 / 98$ \\
\hline \multicolumn{5}{|c|}{ PBS No: RL-TP13 } \\
\hline \multicolumn{2}{|c|}{ MC \#: LLP-98-212 } & TPA NO: & & Rev: 0 \\
\hline $\begin{array}{l}\text { MILESTONE } \\
\text { LEVEL: } \\
\text { DOE-HQ } \\
\text { X DOE-RL } \\
\text { DOE-FO } \\
\text { CONTRACTOR }\end{array}$ & $\begin{array}{l}\text { MILESTONE } \\
\text { TYPE: } \\
\text { EA: } \\
\text { PEG } \\
\text { OTHER }\end{array}$ & $\begin{array}{l}\text { DNFSB STATUS: } \\
\text { DNFSB }(Y / N): \text { : } \\
\text { COMMIT } \#: \\
\text { RECOMM } \#:\end{array}$ & $\begin{array}{l}\text { DELIVERABLE: } \\
\\
\text { Report } \\
\text { Letter } \\
\text { Drawing(s) } \\
\text { O Other (Speci fy) } \\
\text { ACCEPTANCE OF } \\
\text { CONPLETED WORK. } \\
\text {. }\end{array}$ & $\begin{array}{l}\text { ADDRESS TO: } \\
\text { DOE-HQ } \\
\times \text { DOE-RL } \\
\hat{x} \text { Other (Specify) } \\
\text { FDH DIRECTOR OF } \\
\text { INF. \& SEC. }\end{array}$ \\
\hline \multicolumn{5}{|c|}{$\begin{array}{l}\text { Milestone Description: } \\
\text { Completion of installation of an uninterruptable pover supply (UPS) to } \\
\text { provide backup power for vital electrical components within the } 3719 \\
\text { Building. }\end{array}$} \\
\hline \multicolumn{5}{|c|}{$\begin{array}{l}\text { Description of what constitutes completion of this milestone: } \\
\text { The UPS system installed and tested verifying system operability. } \\
\text { Acceptance of Completed Hork to be signed by the following: } \\
\text { DOE-RL: Representative from the Site Infrastructure Division } \\
\text { FOH: } \quad \text { Chief Information officer } \\
\text { FDH: Director of Infrastructure and Security or Designee } \\
\text { Dyncorp: L Landlord Project Technical Representative } \\
\text { LMSI: }\end{array}$} \\
\hline
\end{tabular}




\section{B.3.4 Milestone Description Sheets}

\section{PHMC}

\section{MILESTONE DESCRIPTION SHEET}

\begin{tabular}{|c|c|c|c|c|}
\hline \multicolumn{4}{|c|}{ Title: COMPLETE DESIGN FOR PROJECT L-286. } & Date: $8 / 14 / 97$ \\
\hline \multicolumn{4}{|c|}{ Assigned TO: LANDLORD PROJECT-DYNCORP } & CIN: \\
\hline \multicolumn{4}{|c|}{ Program WBS Designator: 1.5.1.1 } & Due Date: $4 / 24 / 98$ \\
\hline \multicolumn{5}{|c|}{ PBS NO: RL-TP13 } \\
\hline \multicolumn{2}{|c|}{ MC \#: LLP-98-215 } & TPA No: & & Rev: 0 \\
\hline $\begin{array}{l}\text { MILESTONE } \\
\text { LEVEL: } \\
\text { DOE-HQ } \\
\text { XOE-RL } \\
\text { DOE-FO } \\
\text { CONTRACTOR }\end{array}$ & $\begin{array}{l}\text { MILESTONE } \\
\text { TYPE: } \\
\text { EA } \\
\text { PEG } \\
\text { OTHER }\end{array}$ & $\begin{array}{l}\text { DNFSB STATUS: } \\
\text { DNFSB }(Y / N): N \\
\text { COMMIT } \frac{\mathrm{H}}{\pi}: \\
\text { RECONM } \frac{n}{\pi}:\end{array}$ & $\begin{array}{l}\text { DELIVERABLE: } \\
\text { Report } \\
\text { Letter } \\
\text { Drawing(s) } \\
\text { X Other (Specify) } \\
\text { COIJPLETED DESIGN } \\
\text { AND EOT. }\end{array}$ & $\begin{array}{l}\text { ADDRESS TO: } \\
\text { DOE-HQ } \\
\times \text { DOE-RL } \\
\times \text { Other (Specify) } \\
\text { FOH OIRECTOR OF } \\
\text { INF. \& SEC. }\end{array}$ \\
\hline \multicolumn{5}{|c|}{$\begin{array}{l}\text { Milestone Description: } \\
\text { Complete design for Project L-286, '200 East Area Sanitary Water P]ant } \\
\text { Effluent Stream Reduction." }\end{array}$} \\
\hline \multicolumn{5}{|c|}{$\begin{array}{l}\text { Description of what constitutes completion of this milestone: } \\
\text { Approval of Engineering Data Transmittal by the following releasing the } \\
\text { Professional Engineer. stamped drawings and specifications: } \\
\text { DOE-RL: Representative from the Site Infrastructure Division } \\
\text { FDH: } \quad \text { Director of Infrastructure and Security or Designee } \\
\text { DynCorp: Design Authority } \\
\text { DynCorp: Landiord Project Technical Representative }\end{array}$} \\
\hline
\end{tabular}




\section{LANDLORD PROJECT \\ WBS 1.5}

FY 1998 Work Plan

HNF-SP-1242, Rev. 0

B.3.4 Milestone Description Sheets

\section{PHMC}

MILESTONE DESCRIPTION SHEET

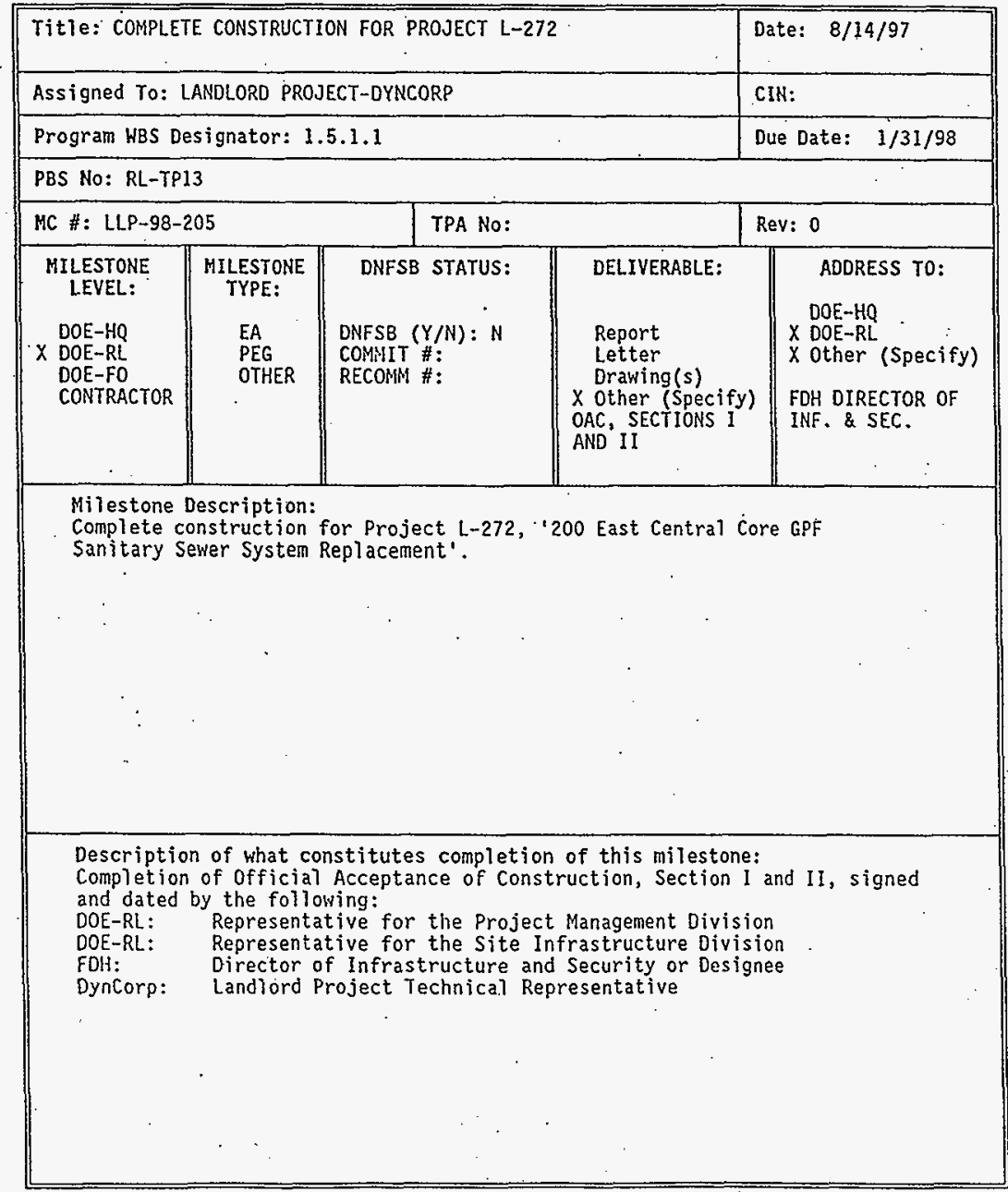




\section{B.3.4 Milestone Description Sheets}

\section{PHMC}

MILESTONE DESCRIPTION SHEET

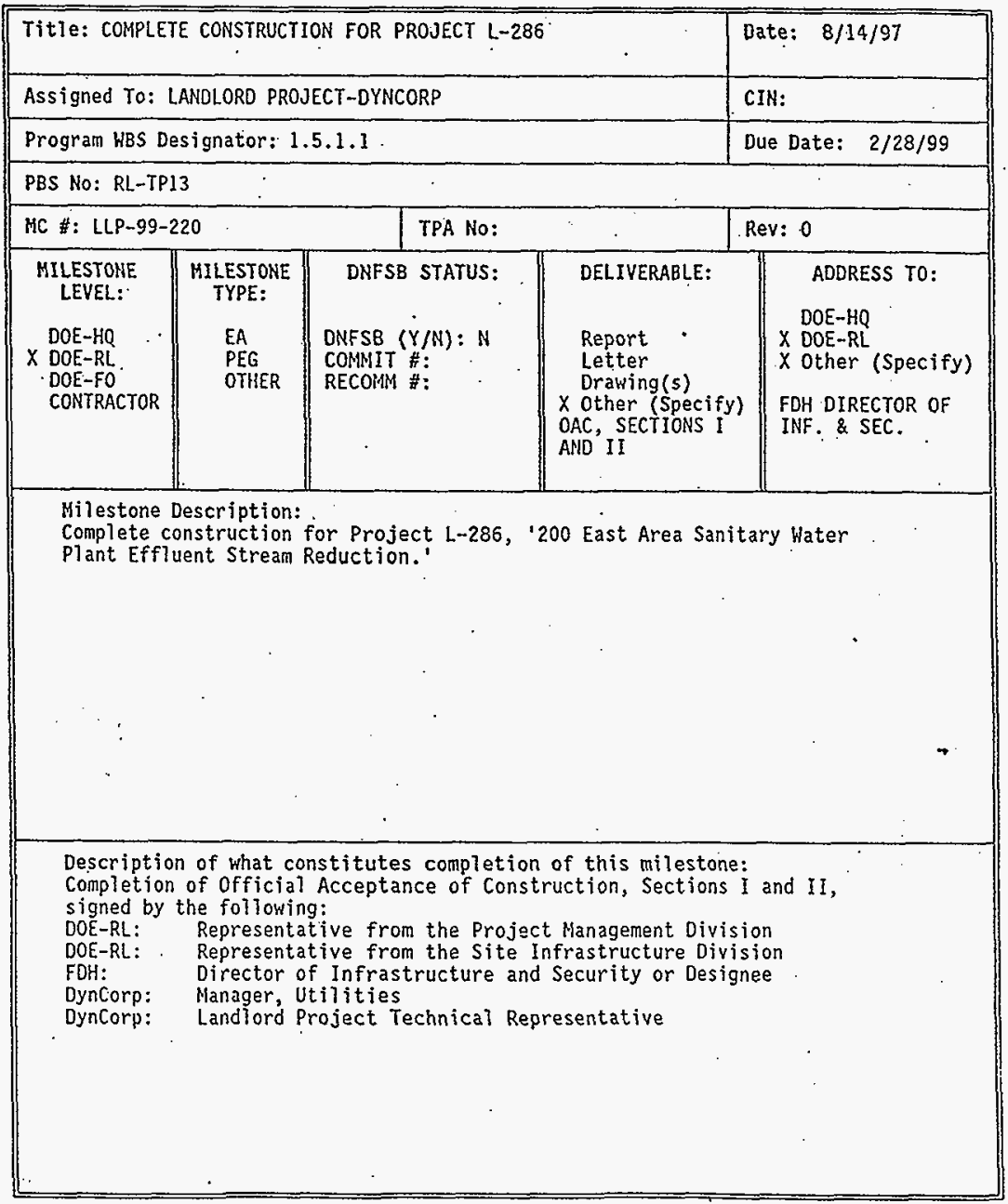




\section{LANDLORD PROJECT \\ WBS 1.5}

FY 1998 Work Plan

HNF-SP-1242, Rev. 0

\section{B.3.4 Milestone Description Sheets}

\section{PHMC}

MILESTONE DESCRIPTION SHEET

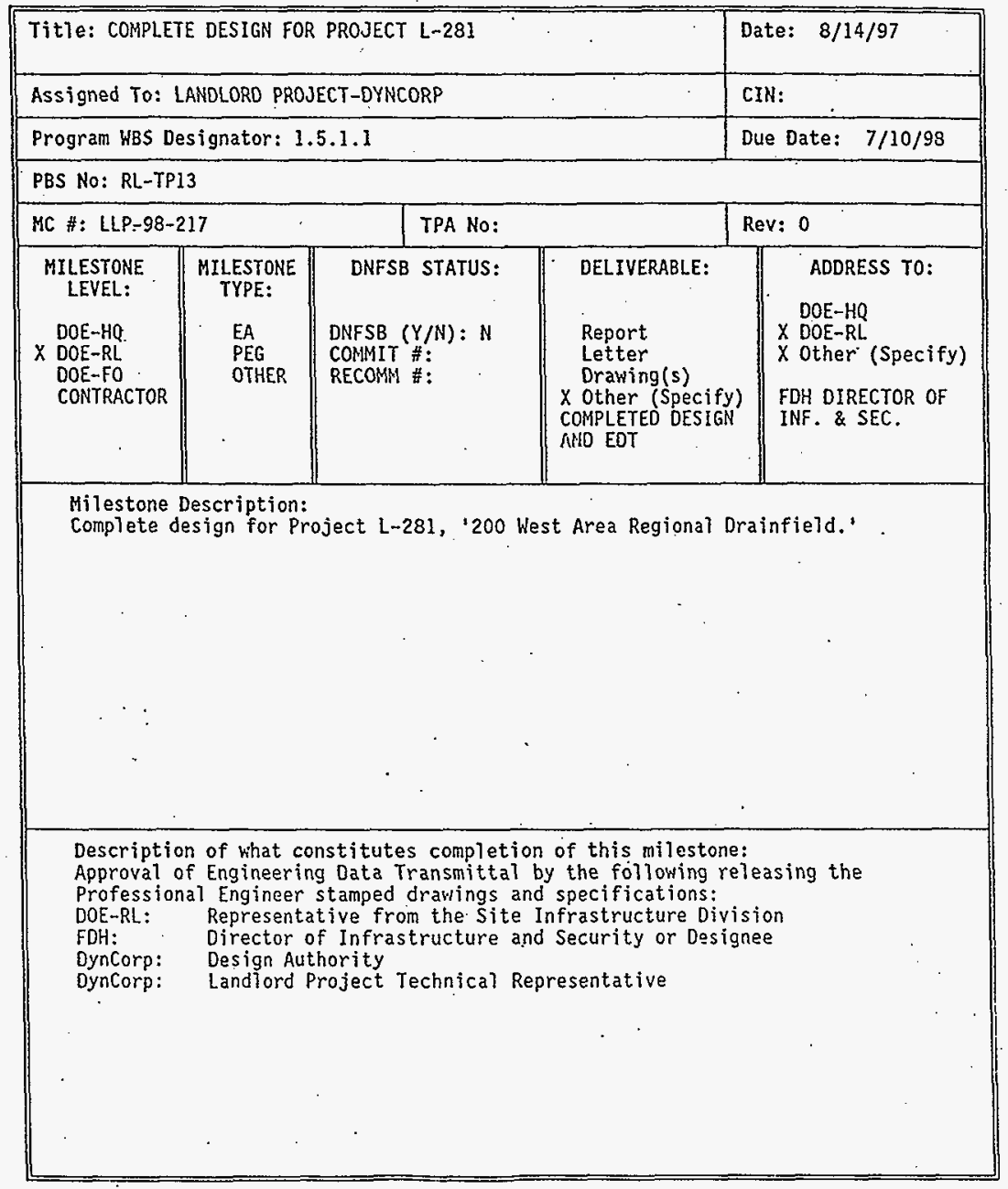




\section{B.3.4 Milestone Description Sheets}

\section{PHMC}

MILESTONE DESCRIPTION SHEET

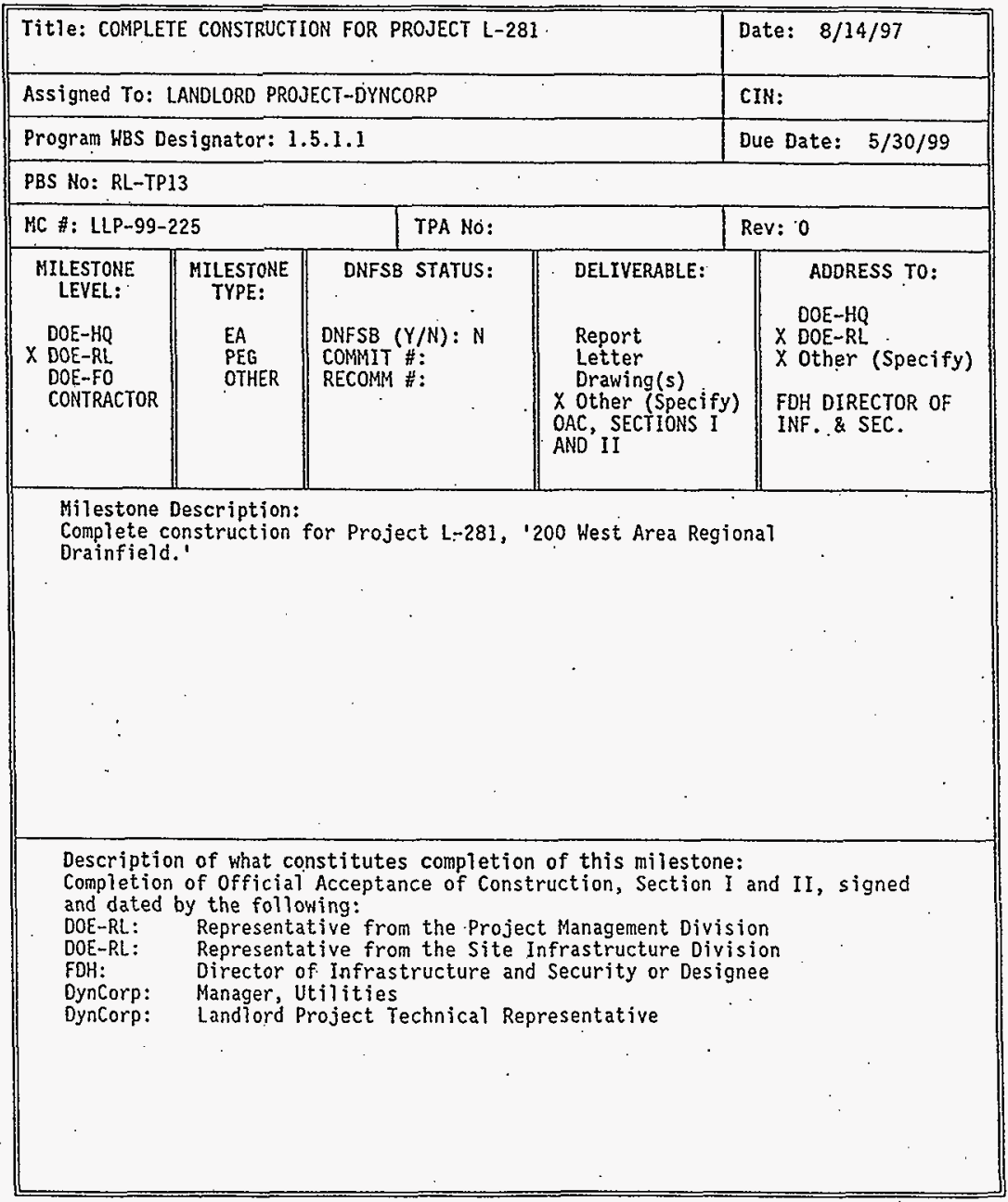




\section{B.3.4 Milestone Description Sheets}

\section{PHMC}

MILESTONE DESCRIPTION SHEET

\begin{tabular}{|c|c|c|c|c|}
\hline \multicolumn{4}{|c|}{$\begin{array}{l}\text { Title: COMPLETE CONSTRUCTION FOR PROJECT B-604, } \\
\text { WATER SYS. UPGRADE-RES. }\end{array}$} & Date: $8 / 14 / 97$ \\
\hline \multicolumn{4}{|c|}{ Assigned TO: LANDLORD PROJECT-DYNCORP } & CIN: \\
\hline \multicolumn{4}{|c|}{ Program WBS Designator: 1.5 .1 .1} & Due Date: $4 / 30 / 97$ \\
\hline \multicolumn{5}{|c|}{ PBS No: RL-TP13 } \\
\hline \multicolumn{3}{|c|}{ MC \#: LLP $-97-020$} & & Rev: 0 \\
\hline $\begin{array}{l}\text { MILESTONE } \\
\text { LEVEL: } \\
\text { DOE-HQ } \\
\text { DOE-RL } \\
\times \text { DOE-FO } \\
\text { CONTRACTOR }\end{array}$ & $\begin{array}{l}\text { MILESTONE } \\
\text { TYPE: } \\
\text { ER } \\
\text { PEG } \\
X \text { OTHER }\end{array}$ & $\begin{array}{l}\text { DNFSB STATUS: } \\
\text { DNFSB }(Y / N): N \\
\text { COMMIT } \#: \\
\text { RECOMM. } \#: \\
:\end{array}$ & $\begin{array}{l}\text { DELIVERABLE: } \\
\text { Report } \\
\text { Letter } \\
\text { Draving( } s \text { ) } \\
\text { X Other (Specify) } \\
\text { OAC, SECIION I }\end{array}$ & $\begin{array}{l}\text { ADDRESS TO: } \\
\text { DOE-HQ } \\
\times \text { DOE-RL } \\
x \text { Other (Specify) } \\
\text { FDH DIRECTOR OF } \\
\text { INF. \& SEC. }\end{array}$ \\
\hline \multicolumn{5}{|c|}{$\begin{array}{l}\text { Milestone Description: } \\
\text { Complete construction for Project 8-604, 'Water System Upgrade -Reservoir.' }\end{array}$} \\
\hline \multicolumn{5}{|c|}{$\begin{array}{l}\text { Description of what constitutes completion of this milestone: } \\
\text { Completion of official Acceptance of Construction, Section I, signed and } \\
\text { dated by the following: } \\
\text { DOE-RL: Representative for the Project Management Division } \\
\text { DOE-RL: Representative for the Site Infrastructure Division } \\
\text { FOH: } \quad \text { Director of Infrastructure and Security or Designee } \\
\text { DynCorp: Landlord Project Technical Representative } \\
\text { Construction is acceptable with noted exceptions that do not preclude the } \\
\text { operations and function of the project. } \\
\qquad\end{array}$} \\
\hline
\end{tabular}




\section{B.3.4 Milestone Description Sheets}

PHMC

MILESTONE DESCRIPTION SHEET

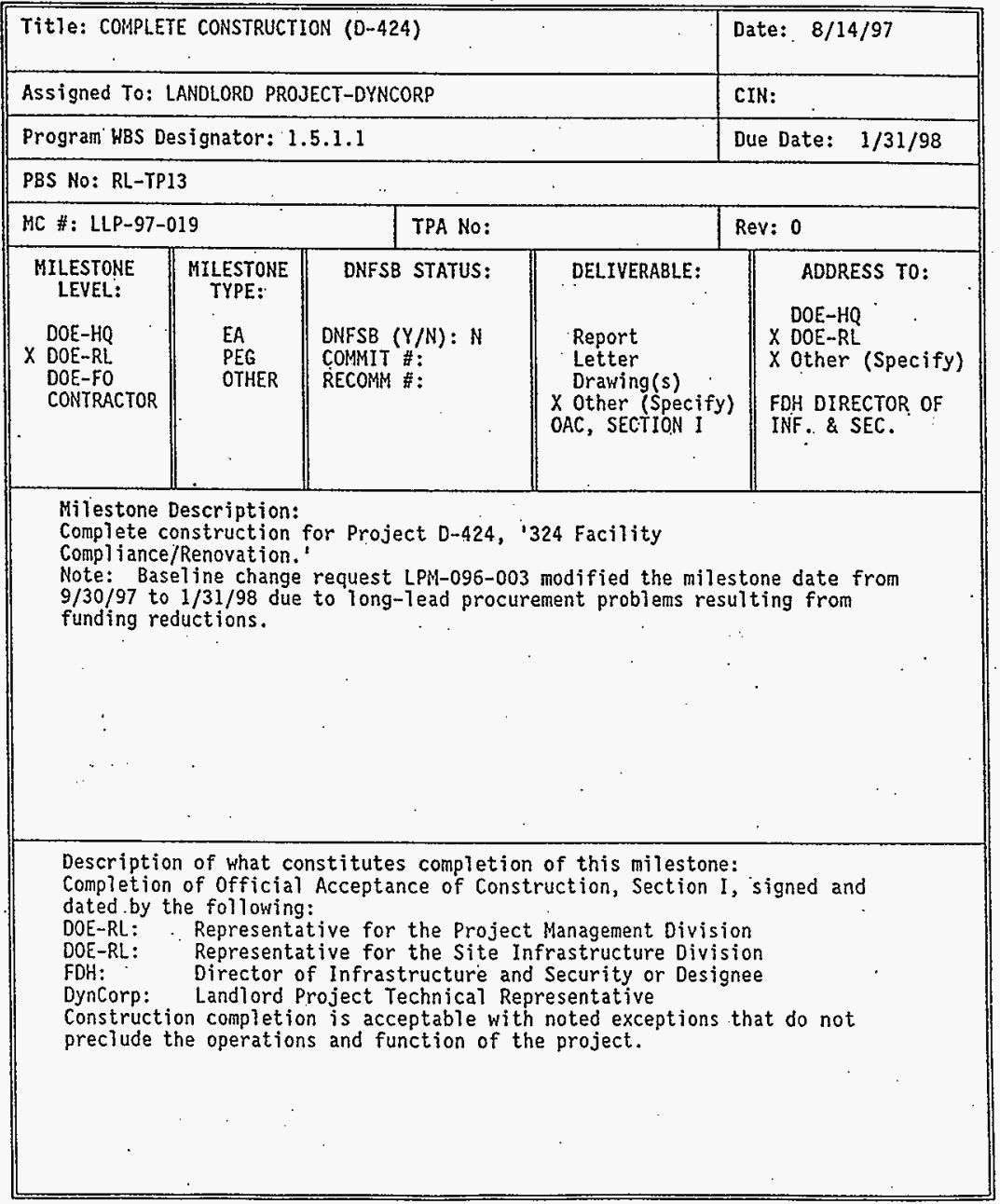


4.0 COST BASELINE 


\section{LANDLORD PROJECT \\ SUMMARY OF LIFE CYCLE COST BASELINE (BCWS). BY YEAR BY BY PROJECT BASELINE SUMMARY (PBS)}

FY 1998

$(\$ 000$ s)

\begin{tabular}{|c|c|c|c|c|c|c|c|c|c|c|c|c|}
\hline \multicolumn{12}{|c|}{ PROJECT WES: } & \multirow{2}{*}{$\begin{array}{l}\text { SUBTOT } \\
\text { FY1997- } \\
\text { FY2006 }\end{array}$} \\
\hline PBS TITLEE & PBS NO & FY1997 & 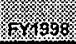 & FY1999 & FY2000 & FY2001 & FY2002 & FY2003 & FY2004 & FY2005 & FY2006 & \\
\hline Landlord Project & RL-TP13 & 11,037 & S & 13,862 & 24,588 & 47,669 & 40,863 & 25,626 & 20.215 & 32.203 & 38,982 & 268,757 \\
\hline & & & 13 & & & & & & & & & - \\
\hline & & & 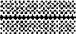 & & & & & & & & & $\div$ \\
\hline & & & 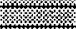 & & & & & & & & & $\cdot$ \\
\hline & & & 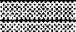 & & & & & & & & & - \\
\hline & & & $\sqrt{2}$ & & & 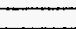 & & & & & & - \\
\hline & & & & & & & & & & & & ت \\
\hline & & & 16. & & & & & & & & & $=$ \\
\hline & & & 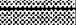 & & & & & & & & & - \\
\hline & & & w & & & & & & & & & \\
\hline 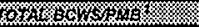 & s) & 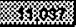 & 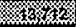 & 㱍筫管 & 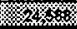 & 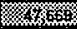 & 2. & 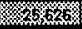 & 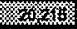 & - & 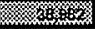 & 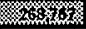 \\
\hline
\end{tabular}

tBudgeted Cost of Work Scheduled (8CWS) Equals Performance Measurement Baseline (PMB); Expense Carryover is NOT Included. 


\section{LANDLORD PROJECT}

SUMMARY OF LIFE CYCLE COST BASELINE (BCWS) BY YEAR BY BY PROJECT BASELINE SUMMARY (PBS)

FY 1998

(\$000s)

\begin{tabular}{|c|c|c|c|c|c|c|c|c|c|c|c|}
\hline \multicolumn{2}{|c|}{ PROJECT WBS: } & \multicolumn{3}{|c|}{1.5} & & & & & & & \\
\hline PBS TITLE & PBS NO & $\begin{array}{l}\text { FY2007- } \\
\text { FY2010 }\end{array}$ & $\begin{array}{l}\text { FY2011- } \\
\text { FY2015 }\end{array}$ & $\begin{array}{l}\text { FY2016- } \\
\text { FY2020 }\end{array}$ & $\begin{array}{l}\text { FY2021- } \\
\text { FY2025 }\end{array}$ & $\begin{array}{l}\text { FY2026- } \\
\text { FY2030 }\end{array}$ & $\begin{array}{l}\text { FY2031- } \\
\text { FY2035 }\end{array}$ & $\begin{array}{l}\text { FY2036 } \\
\text { FY2040 }\end{array}$ & $\begin{array}{l}\text { FY2041- } \\
\text { FY2045 }\end{array}$ & $\begin{array}{l}\text { FY2046 } \\
\text { FY2050 }\end{array}$ & $\begin{array}{l}\text { FY1997- } \\
\text { FY2050 }\end{array}$ \\
\hline Landlord Project & RL-TP13 & 88,000 & 75,000 & 50,000 & 50,000 & 55,550 & 61,000 & 47,700 & 30,329 & 29,900 & 756,235 \\
\hline & & & & & & & & & & & $\cdot$ \\
\hline & & & & & & & & & & & - \\
\hline & & & & & & & & & & & 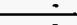 \\
\hline & & & & & & & & & & & $\because$ \\
\hline & & & & & & & & & & & - \\
\hline & & & & & & & & & & & $\dot{-}$ \\
\hline & & & & & & & & & & & - \\
\hline & & & & & & & & & & & $=$ \\
\hline & & & & & & & & & & & $\dot{\square}$ \\
\hline or H & 2 & 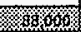 & s. & 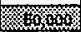 & 8 & 85 & 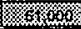 & 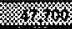 & 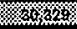 & 然湆晒 & 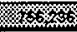 \\
\hline
\end{tabular}


SUMMARY OF LIFE CYCLE BUDGET AUTHORITY (B/A) BY YEAR BY

BY PROJECT BASELINE SUMMARY (PBS)

FY 1998

(\$000s)

\begin{tabular}{|c|c|c|c|c|c|c|c|c|c|c|c|c|}
\hline \multicolumn{3}{|c|}{ PROJECT WBS: 1.5} & & & & & & & & & & \multirow{3}{*}{$\begin{array}{l}\text { SUBTOT } \\
\text { FY1997- } \\
\text { FY2006 } \\
\end{array}$} \\
\hline & & & & & & & & & & & & \\
\hline \multirow[t]{2}{*}{ PBS TITLE } & PBS NO & FY1997 & Fros & FY1999 & FY2000 & FY2001 & FY2002 & FY2003 & FY2004 & FY2005 & FY2006 & \\
\hline & & & 3. & & & & & & & & & - \\
\hline \multirow[t]{12}{*}{\begin{tabular}{|l} 
Landlord Project \\
\end{tabular}} & RL-TP13 & 11,037 & 灌 & 13,357 & 24,588 & 47,669 & 40,863 & 25,626 & 20,215 & 32,203 & 38,982 & 268,254 \\
\hline & & & 弗 & & & & & & & & & - \\
\hline & & & & & & & & & & & & - \\
\hline & & & অ. & & & & & & & & & - \\
\hline & & & & & & & & & & & & . \\
\hline & & & \% & & & & & & & & & $\dot{\theta}$ \\
\hline & & & s & & & & & & & & & . \\
\hline & & & Fis & & & & & & & & &. \\
\hline & & & & & & & & & & & & - \\
\hline & & & 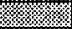 & & & & & & & & & $\dot{\theta}$ \\
\hline & & & 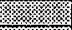 & & & & & & & & & - \\
\hline & & & & & & & & & & & & . \\
\hline & & & & & & & & & & & & \\
\hline 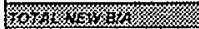 & & 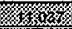 & 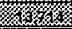 & 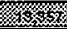 & s. & 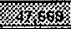 & 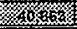 & 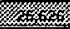 & 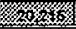 & 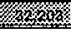 & 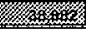 & 落s \\
\hline
\end{tabular}




\begin{tabular}{|c|c|c|c|c|c|c|c|c|c|c|c|}
\hline Sy & (n) & (5) & 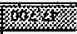 & 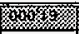 & 2 & 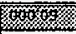 & $300 \%$ & (6) & 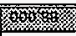 & (1) & 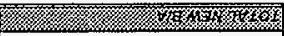 \\
\hline- & & & & & & & & & & & \\
\hline 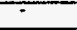 & & & & & & & & & & & \\
\hline$\cdot$ & & & & & & & & & & & \\
\hline$\cdot$ & & & & & & & & & & & \\
\hline - & & & & & & & & & & & \\
\hline - & & & & & & & & & & & \\
\hline$\cdot$ & & & & & & & & & & & \\
\hline$\ddot{*}$ & & & & & & & & & & & \\
\hline - & & & & & & & & & & & \\
\hline$\cdot$ & & & & & & & & & & & \\
\hline$=$ & & & & & & & & & & & \\
\hline- & & & & & & & & & & & \\
\hline Eb9'SSL & $006^{6} 6 \mathrm{Z}$ & $68 z^{\prime} 08$ & $00 L^{\prime} \angle t$ & $000^{\circ} 19$ & OSs'ss & $000^{\circ} 0 \mathrm{~S}$ & $000^{\prime} 0 \mathrm{~s}$ & $000^{\prime} 94$ & $000^{2} 88$ & Eld1-7y & 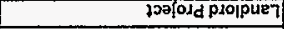 \\
\hline $\begin{array}{l}\text { 0902Ad } \\
\text { - 266thA }\end{array}$ & $\begin{array}{l}\text { 0902Ad } \\
\text { 9rozd }\end{array}$ & $\begin{array}{c}\text { Sp0zAd } \\
-250 z \lambda s\end{array}$ & $\begin{array}{l}\text { 0b02Ad } \\
\text { 980zAd }\end{array}$ & $\begin{array}{r}980 z \lambda A \\
-180 z \lambda A\end{array}$ & $\begin{array}{l}080 z \lambda A \\
-9 z 0 z \lambda A\end{array}$ & $\begin{array}{l}\text { SzozAJ } \\
\text {-LZOZAJ }\end{array}$ & \begin{tabular}{|}
$0202 \lambda]$ \\
$-910 z \lambda A$
\end{tabular} & $\begin{array}{l}\text { SLOZAd } \\
\text { - LLOZAd }\end{array}$ & $\begin{array}{l}0102 \lambda \mathrm{J} \\
-200 \mathrm{KA}\end{array}$ & ON SEd & ᄏ7111 sed \\
\hline $7 \forall 101$ & & & & & & & & & & & \\
\hline
\end{tabular}

(s000\$)

8662 ㅅ

(sQd) ᄉy

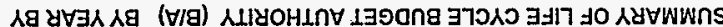
LOتrOYd aYOTaNG7 


\section{LANDLORD PROJECT \\ LIFE CYCLE COST BASELINE (BCWS) BY YEAR BY FUND TYPE BY PROJECT BASELINE SUMMARY (PBS) \\ FY 1998}

\begin{tabular}{|c|c|c|c|c|c|c|c|c|c|c|c|}
\hline $\begin{array}{r}\text { PROJECT WBS: } \\
\text { PBS NO: }\end{array}$ & $\begin{array}{r}1.5 .1 .1 \\
\mathrm{RL} . \mathrm{TP13} \\
\end{array}$ & & & (\$000s) & & & & & & & \\
\hline PBS TITLE: & LANDLORD PROJECT & & & & & & & & & & sUBTor \\
\hline $\begin{array}{l}\text { FUND } \\
\text { TYPE }\end{array}$ & FY1997 & $8 \times 1498$ & FY1999 & FY2000 & FY2001 & FY2002 & FY2003 & Fr2004 & FY2005 & FY2006 & $\begin{array}{l}\text { FY1997- } \\
\text { FY2006 } \\
\end{array}$ \\
\hline OPERATING EXPENSE & 5,989 & \% & 7,925 & 15,568 & 10,669 & 29,863 & 20,126 & 11,715 & 17,203 & 24,982 & 162,688 \\
\hline & & res & & & & & & & & & \\
\hline CENRTC & 1,758 & \% & 2,882 & 4,840 & 2,000 & 5,000 & 2,500 & 3,500 & 5,000 & 6,000 & 35,647 \\
\hline & & 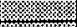 & & & & & & & & & \\
\hline GENERAL PLANT PROJECT & 3,290 & 2188 & 3,055 & 4,180 & 5,000 & 6,000 & 3,000 & 5,000 & 10,000 & 8,000 & 50,013 \\
\hline & & 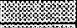 & & & & & & $\because$ & . & 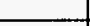 & \\
\hline LINE ITEM (LSA Each OPO) & & 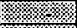 & - & - & - & - & $=$ & $\therefore$ & $\Sigma$ & - & \\
\hline Santary Sclio Wreste Landith Cep & & 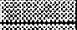 & 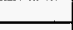 & & 30,000 & 5 & + & + & - & $\cdot$ & 30,000 \\
\hline Project D-424 & & 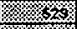 & $=$ & - & - & - & $\dot{.}$ & $\div$ & $\cdot$ & $\therefore$ & 529 \\
\hline & & 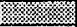 & & & & & & & & & - \\
\hline & & \% & & & & & & & & & - \\
\hline & & \% & & & & & $\therefore$ & & $\therefore$ & & - \\
\hline Subtotal Line ltems & - & \% & - & $\therefore$ & 30,000 & - & + & - & 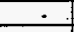 & 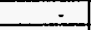 & 30,529 \\
\hline . & & $8 \%$ & & & & & & & & & \\
\hline 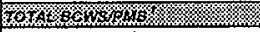 & 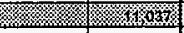 & \% & 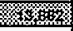 & 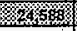 & 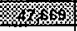 & 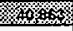 & 2 & 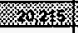 & 8 & 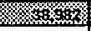 & 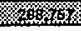 \\
\hline & & 被 & & & & & & & & & \\
\hline MGMT RESERVE ${ }^{2}$ & $=$ & & - & 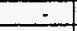 & $\therefore$ & - & $\div$ & - & $\therefore$ & - & $\div$ \\
\hline & & 落觉 & & & & & & & & & \\
\hline LINE ITEM CONTINGENCY ${ }^{2}$ & - & 3.x. & - & - & - & - & 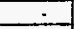 & - & $\therefore$ & - & $=$ \\
\hline & & & & & & & & & & & \\
\hline OFFSITE TRANSFERS $^{3}$ & 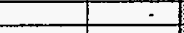 & 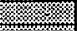 & $\cdot$ & - & - & - & $\div$ & - & $\dot{-}$ & - & - \\
\hline & & & & & & & & & & & \\
\hline Sublotal & $=$ & & $\therefore$ & - & $\dot{-}$ & - & + & $\therefore$ & $\cdot$ & - & 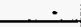 \\
\hline & & 198\% & & & & & & & & & \\
\hline TOTAL & 䈐 & 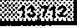 & 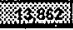 & 2445 & 等 & 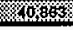 & 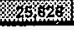 & 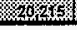 & 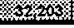 & 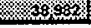 & 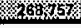 \\
\hline
\end{tabular}

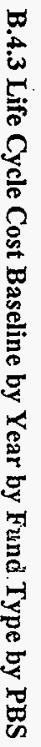

'Budgeted Cost of Work Scheduted (BCWS) Equals Perrormance Measurement Baseline (PMB); Expense Caryover NOT Included. ${ }^{2}$ Management Reserve and Line ltem Contingency Held by RL.

"Work Performed at Sites Other Than Hanford. 
LANDLORD PROJECT

LIFE CYCLE COST BASELINE (BCWS) BY YEAR BY FUND TYPE BY PROJECT BASELINE SUMMARY (PBS)

FY 1998

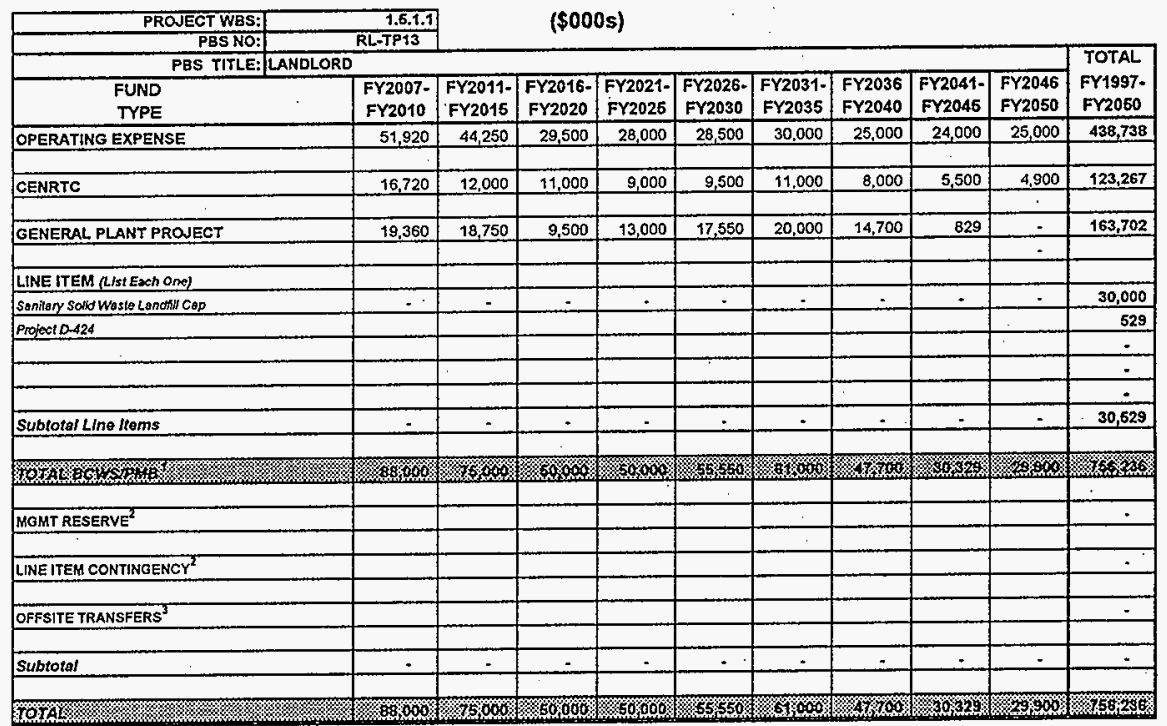


LANDLORD PROJECT

FY 1998 COST BASELINE (BCWS) BY MONTH

BY PROJECT BASELINE SUMMARY (PBS)

BY ACTIVITY DATA SHEET (ADS)

EXECUTION YEAR

(\$000s)

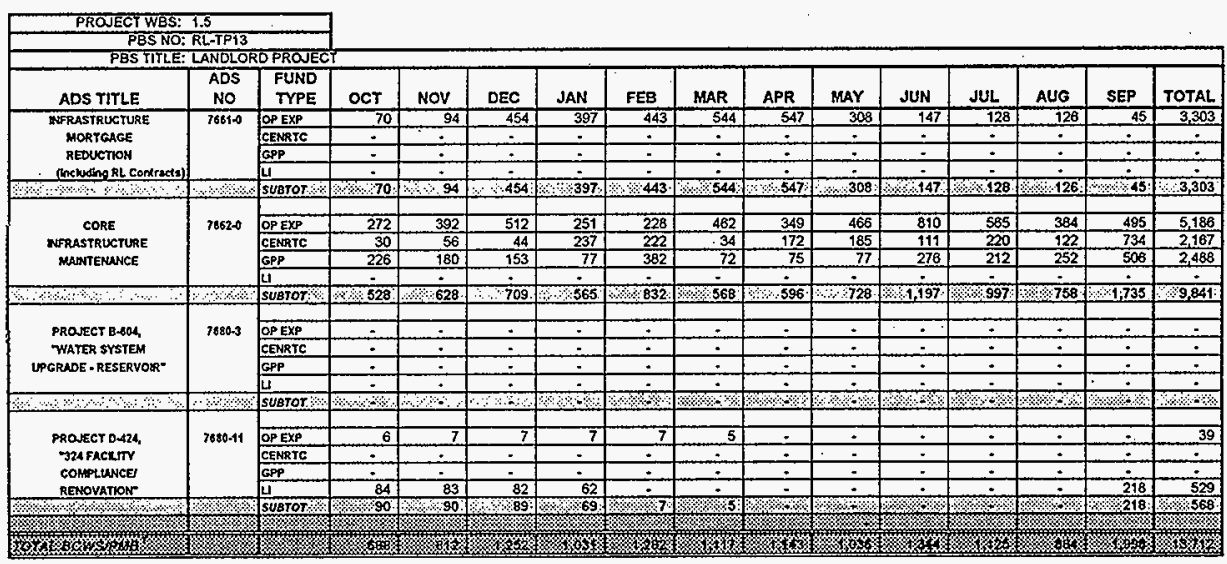




\section{B.4.4.1 FY 1998 Landlord Project Integrated Priority List}

\section{LANDLORD PROJECT INTEGRATED PRIORITY LIST}

\begin{tabular}{|c|c|c|c|c|}
\hline ADS & Description & FY 1998 & $\begin{array}{c}\mathbf{R L} \\
\text { Manager }\end{array}$ & $\begin{array}{c}\text { FDH } \\
\text { Manager }\end{array}$ \\
\hline $7680-11$ & Project D-424, "324 Facility Compliance/Renovation," (Expense Support)" & 39 & Rutherford & Harper \\
\hline $7662-0$ & Program Management* & 720 & Rutherford & Harper \\
\hline $7662-0$ & Land Use Planning/Asset Conversion* & 660 & Rutherford & Harper \\
\hline $7662-0$ & Mapping Services & 432 & Rutherford & Harper \\
\hline $7662-0$ & FDH Feo & 875 & Rutherford & Harper \\
\hline $7662-0$ & Sanitary Solid Waste Landfill Engineering Study (prep for FY 2001 line item) & 130 & Rutherford & Harper \\
\hline $7661-0$ & Radiologically Contaminated Equipment (Railcar Disposition) & 1083 & Rutherford & Harper \\
\hline $7661-0$ & Utilities for Vacant GPFs & 150 & Rutherford & Harper \\
\hline $7662-0$ & Med 94, 68G-3860/G31 12907 Advanced Life Support Ambulance (1989) & 175 & Veitenheimer & Harper \\
\hline $7662-0$ & Overlay/Seal Roads & 1041 & Rutherford & Harper \\
\hline $7661-0$ & Vacant GPF Facilities Demolition & 0 & Rutherford & Harper \\
\hline $7662-0$ & Area Emergency Sirens, Phase III & 830 & Veitenheimer & Harper \\
\hline $7662-0$ & Mobile Tactical incident Command Unit & 0 & Veitenheimer & Harper \\
\hline $7661-0$ & Surveillance \& Maintenance of GPFs & 170 & Rutherford. & Harper \\
\hline $7662-0$ & Project L-279, "Outer Area Fiber Optic Loop" & 979 & Rutherford & Sterling \\
\hline $7662-0$ & L-286, "200 East Area Sanitary Water Plant Effluent Stream Reduction" & 1430 & Rutherford & Harper \\
\hline $7662-0$ & UPS for 3719 Building & 225 & Rutherford & Sterling \\
\hline $7662-0$ & Emergency Services Personnel Consolidation (Project L-275) & 849 & Veitenheimer & Harper \\
\hline $7662-0$ & Emergency Services Vehicles Consolidation (Project L-276) & 0 & Veitenheimer & Harper \\
\hline $7662-0$ & Mult-Plex Emergency Radio Base Station & 135 & Veitenheimer & Harper \\
\hline $7662-0$ & L-281, "200 West Area Regional Drainfield" & $\$ 126$ & Rutherford & Harper \\
\hline $7662-0$ & Final Regulatory Disposition of 300 Area Abandoned Septic System & 79 & Rutherford & Harper \\
\hline $7662-0$ & PNNL Computer Replacement for Radiological Assessments & 40 & Roselli & Harper \\
\hline $7661-0$ & Shutdown of Remaining Steam Plants (284E and 384) & 1900 & Rutherford & Harper \\
\hline $7661-0$ & Isolation/Shutdown/Maintenance of Vacant GPF Facilities & 0 & Rutherford & Harper \\
\hline $7662-0$ & Final Regulatory Disposition of Outer Area Abandoned Septic System & 111 & Rutherford & Harper \\
\hline $7662-0$ & Engine \#91, 68D-3871, Fire Engine Aerial Ladder 50' (1978) & 535 & Veitenheimer & Harper \\
\hline & FY 1998 Funding Level & $\overline{13714}$ & & \\
\hline
\end{tabular}




\section{PROJECT HANFORD BREAKDOWN STRUCTURE LANDLORD PROJECT}

3 DIGIT CONTRACTOR IDENTIFIER-PR-001

Revision 0

AVERAGE ANNUAL FULLL TIME EQUIVALENTS

(includes Major Subcontractors but not Enterprise Companies)

PHBS 1.5

Submittal Date: September 1997

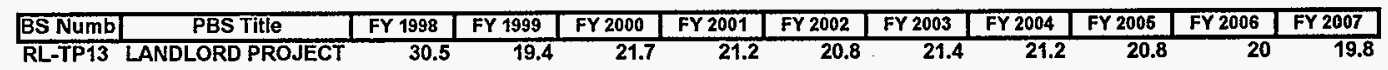




\section{LANDLORD PROJECT WBS 1.5}

FY 1998 Work Plan

HNF-SP-1242, Rev. 0

\section{B.4.5 Staffing}

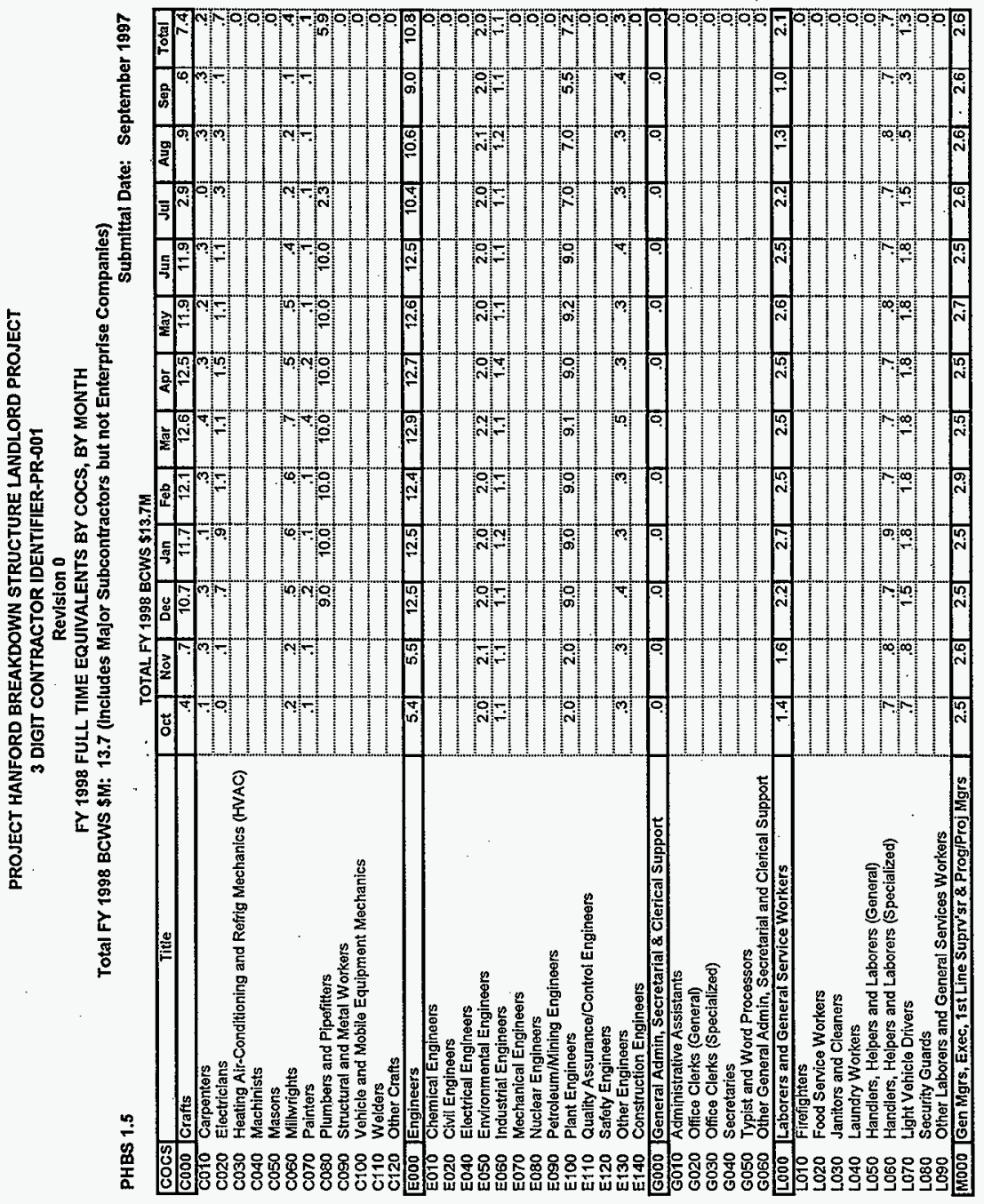


LANDLORD PROJECT

WBS 1.5

FY 1998 Work Plan

HNF-SP-1242, Rev. 0

\section{B.4.5 Staffing}

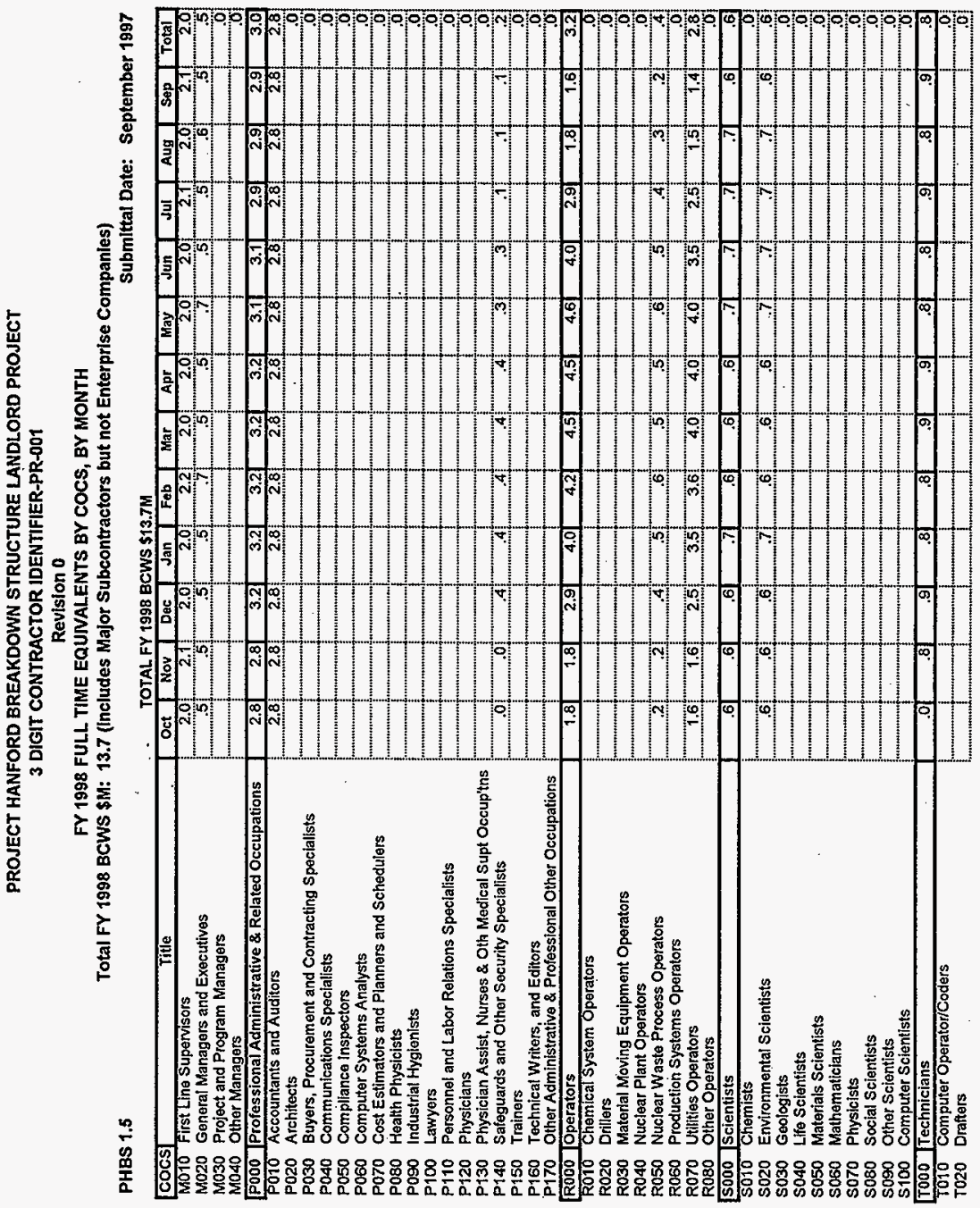


PROJECT HANFORD BREAKDOWN STRUCTURE LANDLORD PROJECT 3 DIGIT CONTRACTOR IDENTIFIER-PR-001

Revision 0

FY 1998 FULL TIME EQUNALENTS BY COCS, BY MONTH

PHBS 1.5

Total FY 1998 BCWS \$M: 13.7 (Includes Major Subcontractors but not Enterprise Companies)

Submittal Date: September 1997

\begin{tabular}{|c|c|c|c|c|c|c|c|c|c|c|c|c|c|c|}
\hline COCS & Title & Oet & Nov & Dec & $\operatorname{Jan}$ & Feb & Mar & Apr & May & Jun & Jul & Aug & Sep & Total \\
\hline T030 & Engineering Technicians & & & & & & & & & & & & & .0 \\
\hline T040 & Environmental Selences Technleians & & & & & & & & & & & & & 0 \\
\hline T050 & Health Physies Technicians & .0 & .8 & .9 & .8 & .8 & 9 & .9 & .8 & 8 & .9 & .8 & 9 & .8 \\
\hline T060 & Industrial Safety and Health Tochnicians & & & & & & & & & & & & & .0 \\
\hline T070 & Instrument and Control Technicians & & & & & & & & & & & & & .0 \\
\hline T080 & Laboratory Technicians & & & & & & & & & & & & & .0 \\
\hline T090 & Modia Technicians & & & & & & & & & & & & & 0 \\
\hline T100 & Survey and Mapping & 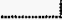 & 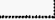 & & & & "מ.: & 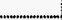 & & 1 & & & & 0 \\
\hline T110 & Other Technicians & & & & & & & & & & & & & 0 \\
\hline Total & & 14.9 & 16.4 & 35.5 & 38.1 & 38.7 & 39.7 & 39.4 & 39.0 & 38.0 & 25.5 & 21.6 & 19.2 & 30.5 \\
\hline
\end{tabular}


PROJECT HANFORD BREAKDOWN STRUCTURE LANDLORD PROJECT

3 DIGIT CONTRACTOR IDENTIFIER-PR-001

Revision 0

TEN YEAR FULL TIME EQUIVALENTS BY COCS

PHBS 1.5

(includes Major Subcontractors but not Enterprise Companies)

Each Year's BCWs \$M

\begin{tabular}{|c|c|c|}
\hline COCS & \\
\hline Co00 & Cratis \\
\hline
\end{tabular}

(includes Major Subcontractors but not Enterprise Companies

Submittal Date: September 1997

CO10 Carpenters

CO20 Electricians

C030 Heating Air-Conditioning and Refrig Mechanics (HVAC)

CO40 Machinists

CO50 Masons

CO60 Millwights

co70 Paintors

coso Plumbers and Pipefitters

C090 Structural and Metal Workers

C100 Vehicle and Mobile Equipment Mechanics

C110 Welders

C120 Other Crafts

E000 Engineers

E010 Chemical Engineers

E020 Civil Engineers

E040 Electrical Engineers

E050 Environmental Engineers

E060 Industrial Engineers

E070 Mechanical Engineers

E080 Nuclear Engineers

E090 Petroleum/Mining Engineers

E100 Plant Englneers

E110 Quality Assurance/Control Englneers

E120 Safety Engineers

E130 Other Engineers

E140 Construction Enginoors

G000 General Admin, Secretarial \& Clerical Support

G010 Administrative Assistants

G020. Office Clerks (General)

$\mathrm{G} 030$ Office Clerks (Speclalized)

G040 Secretaries

G050 Typist and Word Processors

G060 Other General Admin, Secretarial and Clerical Support

LO00 Laborers and General Service Workers

Loto Fitefighters

L020 Food Service Workers

L030 Janitors and Cleaners

L040 Laundry Workers

L050 Handlers, Helpers and Laborers (General)

L060 Handlers, Helpers and Laborers (Specialized)

L070 Light Vehicle Drivers

$\begin{array}{llllllllll}\$ 13.7 & \$ 13.4 & \$ 22.0 & \$ 22.0 & \$ 22.0 & \$ 22.0 & \$ 22.0 & \$ 22.0 & \$ 22.0 & \$ 22.0\end{array}$ \begin{tabular}{|r|r|r|r|r|r|r|r|r|r|}
\hline FY 1998 & FY 1999 & FY 2000 & FY 2001 & FY 2002 & FY 2003 & FY 2004 & FY 2005 & FY 2006 & FY 2007 \\
\hline
\end{tabular}

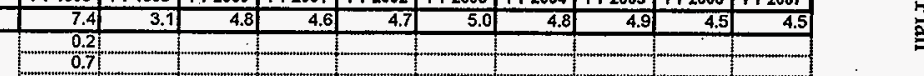




\section{B.4.5 Staffing}

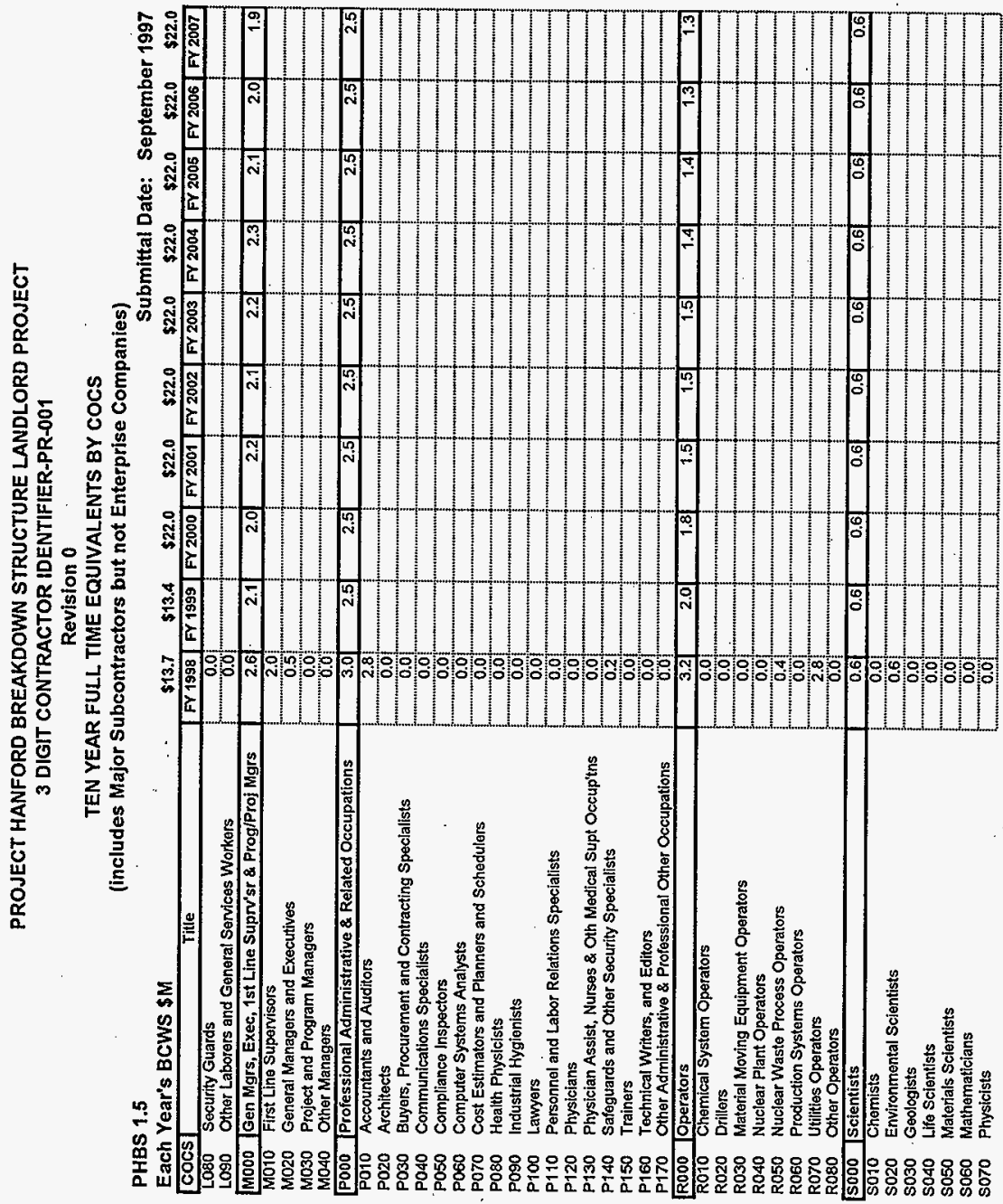


PROJECT HANFORD BREAKDOWN STRUCTURE LANDLORD PROJECT 3 DIGIT CONTRACTOR IDENTIFIER-PR-001

Revision 0

TEN YEAR FULLL TIME EQUIVALENTS BY COCS

PHBS 1.5

(includes Major Subcontractors but not Enterprise Companies)

Each Year's BCWS $\$ M$

\begin{tabular}{|c|c|c|c|c|c|c|c|c|c|c|c|}
\hline Each & Year's BCWS \$M & $\$ 13.7$ & $\$ 13.4$ & $\$ 22.0$ & $\$ 22.0$ & $\$ 22.0$ & $\$ 22.0$ & $\$ 22.0$ & $\$ 22.0$ & $\$ 22.0$ & $\$ 22.0$ \\
\hline COCS & 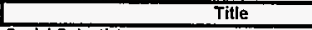 & FY 4998 & FY1999 & FY2000 & FY 2001 & Fr2002 & FY2003 & FY2004 & FY 2005 & FY2006 & FY2007 \\
\hline 5080 & Social Scientists & 0.0 & & & & & & & & & \\
\hline $\begin{array}{l}s 090 \\
\$ 100\end{array}$ & $\begin{array}{l}\text { Other Scientists } \\
\text { Computer Scientists }\end{array}$ & 0.0 & & & & & & & & & \\
\hline T000 & $\begin{array}{l}\text { Computer Scientists } \\
\text { Technicians }\end{array}$ & $\frac{0.01}{0.8}$ & & & & & & & & & \\
\hline T010 & Computer Operator/Coders & 0.8 & 0.8 & 0.8 & 0.7 & 0.7 & 0.7 & 0.6 & 0.5 & 0.6 & 0.5 \\
\hline TO20 & Drafters & 0.0 & & & & & & & & & \\
\hline T030 & Engineoring Technicians & 0.0 & & & & & & & & & \\
\hline T040 & Environmental Sciences Technicians & 0.0 & & & & & & & & & \\
\hline $\operatorname{TOSO}$ & Health Physics Technicians & 0.8 & & & & & & & & & \\
\hline$T 060$ & Industrial Safety and Health Technicians & 0.0 & & & & & & & & & \\
\hline TO70 & $\begin{array}{l}\text { Instrument and Control Techniclans } \\
\text { Laboratory Techniclans }\end{array}$ & 0.0 & & & & & & & & & \\
\hline TO80 & $\begin{array}{l}\text { Laboratory Techniclans } \\
\text { Media Technicians }\end{array}$ & 0.0 & & & & & & & & & \\
\hline TO90 & Media Technicians & 0.0 & & & & & & & & & \\
\hline T100 & $\begin{array}{l}\text { Suvey and Mapping } \\
\text { Other Techniclans }\end{array}$ & 0.0 & & & & & & & & & \\
\hline Total & & 30.5 & 19.4 & 21.7 & 21.2 & 20.8 & $\frac{i}{21.4}$ & 21.2 & 20.8 & 20.0 & 198 \\
\hline
\end{tabular}



LANDLORD PROJECT

WBS 1.5

FY 1998 Work Plan

HNF-SP-1242, Rev. 0

\section{HANFORD SITE WORK BREAKDOWN STRUCTURE DICTIONARY \\ LANDLORD}

WBS: 1.5

\begin{tabular}{|l|l|l|c|}
\hline $\begin{array}{l}\text { 1. Activity Title: } \\
\text { Infrastructure Mortgage Reduction - } \\
\text { Administration }\end{array}$ & $\begin{array}{l}\text { 2. Date } \\
\text { July } 1997\end{array}$ & $\begin{array}{l}\text { 3. PBS Number } \\
\text { RL-TP13 }\end{array}$ & $\begin{array}{c}\text { 4. Dict. Rev. } \\
0\end{array}$ \\
\hline 5. Contract WBS No. & $\begin{array}{l}\text { 6. Corresponding } \\
\text { FDS No. }\end{array}$ & 7. Baseline CR No. \\
\hline $\begin{array}{l}\text { 8.5.1.1.1.1 } \\
\text { DynCorganization Name Operations }\end{array}$ & $\begin{array}{l}\text { 9. B \& R No. } \\
\text { EW7070201 }\end{array}$ \\
\hline
\end{tabular}

10. Scope of Work

This workscope would include administrative activities associated with reducing site mortgages, such as monitoring, planning, reporting and baseline management. 


\section{LANDLORD PROJECT \\ WBS 1.5}

FY 1998 Work Plan

HNF-SP-1242, Rev. 0

\section{HANFORD SITE WORK BREAKDOWN STRUCTURE DICTIONARY \\ LANDLORD}

WBS: 1.5

\begin{tabular}{|l|l|l|c|}
\hline $\begin{array}{l}\text { 1. Activity Title: } \\
\begin{array}{l}\text { Infrastructure Mortgage Reduction - } \\
\text { Business Systems }\end{array}\end{array}$ & $\begin{array}{l}\text { 2 Date } \\
\text { July } 1997\end{array}$ & $\begin{array}{l}\text { 3. PBS Number } \\
\text { RL-TP13 }\end{array}$ & $\begin{array}{c}\text { 4. Dict. Rev. } \\
0\end{array}$ \\
\hline $\begin{array}{l}\text { 5. Contract WBS No. } \\
\text { 1.5.1.1.1.2 }\end{array}$ & $\begin{array}{l}\text { 6. Corresponding } \\
\text { FDS No. }\end{array}$ & $\begin{array}{l}\text { 7. Baseline CR No. } \\
\text { 8. Organization Name } \\
\text { DynCorp Operations }\end{array}$ & $\begin{array}{l}\text { 9. B \& R No. } \\
\text { EW7070201 }\end{array}$ \\
\hline
\end{tabular}

10. Scope of Work

This workscope would include projects related to reducing site mortgages by replacing business systems and components that are antiquated and expensive to maintain. Where possible, this equipment would be replaced by energy saving equipment, thereby saving the entire site incremental costs.

Currentiy, there are no planned projects in FY 1998. 


\section{LANDLORD PROJECT \\ WBS 1.5}

FY 1998 Work Plan

HNF-SP-1242, Rev. 0

HANFORD SITE WORK BREAKDOWN STRUCTURE DICTIONARY

LANDLORD

WBS: 1.5

\begin{tabular}{|l|l|l|c|}
\hline $\begin{array}{l}\text { 1. Activity Title: } \\
\text { Infrastructure Mortgage Reduction - } \\
\text { Sewer }\end{array}$ & $\begin{array}{l}\text { 2 Date } \\
\text { July } 1997\end{array}$ & $\begin{array}{l}\text { 3. PBS Number } \\
\text { RL-TP13 }\end{array}$ & $\begin{array}{c}\text { 4. Dict. Rev. } \\
0\end{array}$ \\
\hline $\begin{array}{l}\text { 5. Contract WBS No. } \\
\text { 1.5.1.1.1.3 }\end{array}$ & $\begin{array}{l}\text { 6. Corresponding } \\
\text { FDS No. }\end{array}$ & $\begin{array}{l}\text { 7. Baseline CR No. } \\
\text { 8. Organization Name } \\
\text { DynCorp Operations }\end{array}$ & $\begin{array}{l}\text { 9. B \& R No. } \\
\text { EW7070201 }\end{array}$ \\
\hline
\end{tabular}

10. Scope of Work

This workscope would include projects related to reducing site mortgages by replacing sewer equipment and components that are antiquated and expensive to maintain. Where possible, this equipment would be replaced by energy saving equipment, thereby saving the entire site incremental costs.

Currently, there are no planned projects in FY 1998. 


\section{LANDLORD PROJECT \\ WBS 1.5 .}

FY 1998 Work Plan

HNF-SP-1242, Rev. 0

\section{HANFORD SITE WORK BREAKDOWN STRUCTURE DICTIONARY}

LANDLORD

WBS: 1.5

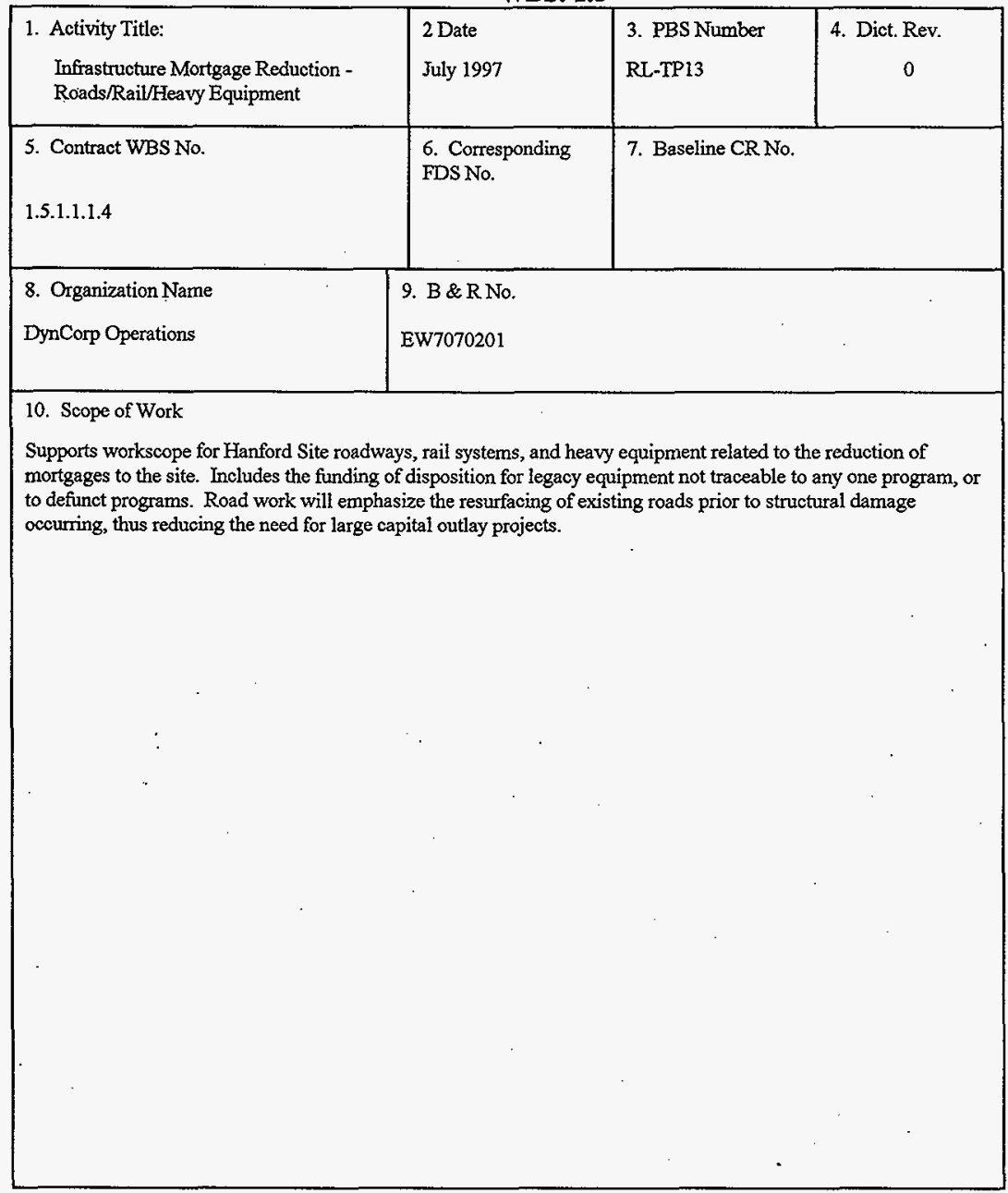




\section{HANFORD SITE WORK BREAKDOWN STRUCTURE DICTIONARY}

\section{LANDLORD}

WBS: 1.5

\begin{tabular}{|c|c|c|c|}
\hline $\begin{array}{l}\text { 1. Activity Title: } \\
\text { Infrastructure Mortgage Reduction - } \\
\text { Equipment Disposition }\end{array}$ & $\begin{array}{l}\text { 2Date } \\
\text { July } 1997\end{array}$ & $\begin{array}{l}\text { 3. PBS Number } \\
\text { RL-TP13 }\end{array}$ & $\begin{array}{c}\text { 4. Dict. Rev. } \\
0\end{array}$ \\
\hline $\begin{array}{l}\text { 5. Contract WBS No. } \\
\text { 1.5.1.1.1.4A }\end{array}$ & $\begin{array}{l}\text { 6. Corresponding } \\
\text { FDS No. }\end{array}$ & \multicolumn{2}{|l|}{ 7. Baseline CR No. } \\
\hline $\begin{array}{l}\text { 8. Organization Name } \\
\text { DynCorp Operations }\end{array}$ & \multicolumn{3}{|l|}{$\begin{array}{l}\text { 9. B \& RNo. } \\
\text { EW7070201 }\end{array}$} \\
\hline \multicolumn{4}{|c|}{$\begin{array}{l}\text { 10. Scope of Work } \\
\text { This scope dispositions RCRA non-compliant and radiologically contaminated rail equipment managed by the } \\
\text { contractor for DOE-RL. The objective of the project is to bring the non-compliant equipment in compliance with } \\
\text { RCRA regulations and to develop and implement the final dispositioning of these equipment pieces. }\end{array}$} \\
\hline
\end{tabular}




\section{HANFORD SITE WORK BREAKDOWN STRUCTURE DICTIONARY LANDLORD \\ WBS: 1.5}

\begin{tabular}{|l|l|l|c|}
\hline $\begin{array}{l}\text { 1. Activity Title: } \\
\text { Infrastructure Mortgage Reduction - } \\
\text { Electrical Distribution }\end{array}$ & $\begin{array}{l}\text { Date } \\
\text { July } 1997\end{array}$ & $\begin{array}{l}\text { 3. PBS Number } \\
\text { RL-TP13 }\end{array}$ & $\begin{array}{c}\text { 4. Dict. Rev. } \\
0\end{array}$ \\
\hline 5. Contract WBS No. & $\begin{array}{l}\text { 6. Corresponding } \\
\text { FDS No. }\end{array}$ & $\begin{array}{l}\text { 7. Baseline CR No. } \\
\text { 1.5.1.1.1.5 }\end{array}$ & $\begin{array}{l}\text { 9. B \& R No. } \\
\text { 8. Organization Name }\end{array}$ \\
DynCorp Operations & EW7070201 & \\
\hline
\end{tabular}

10. Scope of Work

This workscope would include projects related to reducing site mortgages by replacing electrical distribution equipment that is antiquated and expensive to maintain. Where possible, this equipment would be replaced by energy saving equipment, thereby saving the entire site incremental costs. Also, this activity would include the removal of legacy equipment and cable which would be sold for salvage value.

Currently, there are no planned projects in FY 1998. 
HANFORD SITE WORK BREAKDOWN STRUCTURE DICTIONARY LANDLORD

WBS: 1.5

\begin{tabular}{|c|c|c|c|}
\hline $\begin{array}{l}\text { 1. Activity Title: } \\
\text { Infrastructure Mortgage Reduction - } \\
\text { Emergency Facilities/Services }\end{array}$ & $\begin{array}{l}2 \text { Date } \\
\text { July } 1997\end{array}$ & $\begin{array}{l}\text { 3. PBS Number } \\
\text { RL-TP13 }\end{array}$ & $\begin{array}{c}\text { 4. Dict. Rev. } \\
0\end{array}$ \\
\hline $\begin{array}{l}\text { 5. Contract WBS No. } \\
\text { 1.5.1.1.1.6 }\end{array}$ & $\begin{array}{l}\text { 6. Corresponding } \\
\text { FDS No. }\end{array}$ & \multicolumn{2}{|c|}{ 7. Baseline CR No. } \\
\hline $\begin{array}{l}\text { 8. Organization Name } \\
\text { DynCorp Operations }\end{array}$ & \multicolumn{3}{|l|}{$\begin{array}{l}\text { 9. B \& RNo. } \\
\text { EW7070201 }\end{array}$} \\
\hline \multicolumn{4}{|c|}{$\begin{array}{l}\text { 10. Scope of Work } \\
\text { This workscope would include projects related to reducing site mortgages by replacing emergency facilities/services } \\
\text { equipment and components that are antiquated and expensive to maintain. Where possible, this equipment would be } \\
\text { replaced by energy saving equipment, thereby saving the entire site incremental costs. } \\
\text { Currently, there are no planned projects in FY } 1998 \text {. }\end{array}$} \\
\hline
\end{tabular}




\section{HANFORD SITE WORK BREAKDOWN STRUCTURE DICTIONARY LANDLORD}

WBS: 1.5

\begin{tabular}{|l|l|l|c|}
\hline $\begin{array}{l}\text { 1. Activity Title: } \\
\begin{array}{l}\text { Infrastructure Mortgage Reduction - } \\
\text { Facilities/Shops/PNNL Laboratories }\end{array}\end{array}$ & $\begin{array}{l}\text { 2Date } \\
\text { July } 1997\end{array}$ & $\begin{array}{l}\text { 3. PBS Number } \\
\text { RL-IP13 }\end{array}$ \\
\hline 5. Contract WBS No. & $\begin{array}{l}\text { 6. Corresponding } \\
\text { FDS No. }\end{array}$ & $\begin{array}{l}\text { 7. Baseline CR No. } \\
\text { 1.5.1.1.1.7 }\end{array}$ & $\begin{array}{l}\text { 9. B \& R No. } \\
\text { EW7070201 }\end{array}$ \\
\hline $\begin{array}{l}\text { 8. Organization Name } \\
\text { DynCorp Operations }\end{array}$ & \\
\hline
\end{tabular}

10. Scope of Work

This workscope includes mortgage reduction activities to reduce the number of occupied general purpose facilities on the Hanford Site. This includes isolating the facilities from all utilities in a cost cutting effort, thereby reducing the necessary surveillance costs. In cases where the fire protection systems must be maintained after the facility is vacated, the utility costs are included in this activity. The buildings can then be transitioned to the demolition program or can be redeployed to private entities. 


\section{HANFORD SITE WORK BREAKDOWN STRUCTURE DICTIONARY LANDLORD}

WBS: 1.5

\begin{tabular}{|l|l|l|c|}
\hline $\begin{array}{l}\text { 1. Activity Title: } \\
\begin{array}{l}\text { Infrastructure Mortgage Reduction - } \\
\text { Utilities, Surveillance and Maintenance }\end{array}\end{array}$ & $\begin{array}{l}\text { 2 Date } \\
\text { July } 1997\end{array}$ & $\begin{array}{l}\text { 3. PBS Number } \\
\text { RL-TP13 }\end{array}$ & 0 \\
\hline 5. Contract WBS No. & $\begin{array}{l}\text { 6. Corresponding } \\
\text { FDS No. }\end{array}$ & $\begin{array}{l}\text { 7. Baseline CR No. } \\
\text { 1.5.1.1.1.7A }\end{array}$ & 9. B \& R No. \\
\hline \begin{tabular}{l|l} 
8. Organization Name \\
DynCorp Operations
\end{tabular} & EW7070201 & \\
\hline
\end{tabular}

10. Scope of Work

This WBS of the Hanford Landlord Program provides for the shutdown and isolation of unoccupied general purpose facilities. This WBS also includes a portion of the utility costs for vacant facilities required to maintain fire protection systems. This project is driven by the site mission of cleanup, federal and state laws concerning management and exposure to hazardous materials and conditions (OSHA 29 Code of Federal Regulations 1926.58 and Washington Administrative Code 296-62-077), and transition of real estate for other uses. The infrastructure age and initial "temporary" nature of facilities to support the Manhattan Project has resulted in generally deteriorated conditions and inefficient systems. This activity supports providing a safe workplace that is free from fatalities and serious accidents, and continuously reduces injuries and adverse health effects. 


\section{HANFORD STTE WORK BREAKDOWN STRUCTURE DICTIONARY LANDLORD}

WBS: 1.5

\begin{abstract}
1. Activity Title:
Infrastructure Mortgage Reduction Shutdown of Remaining Steam Plants
\end{abstract}

5. Contract WBS No.

1.5.1.1.1.7C

8. Organization Name

DynCorp Operations
9. $B \& R N o$.

EW7070201

10. Scope of Work

Shutdown and isolation of the $284 \mathrm{E}$ and 384 Steam Plants supports the award of the energy savings performance contract. These facilities will be shutdown and isolated in FY 1998 to support demolition at a later date. 


\section{HANFORD SITE WORK BREAKDOWN STRUCTURE DICTIONARY LANDLORD \\ WBS: 1.5}

\begin{tabular}{|l|l|l|c|}
\hline $\begin{array}{l}\text { 1. Activity Title: } \\
\begin{array}{l}\text { Infrastructure Mortgage Reduction - } \\
\text { Telecommunications }\end{array}\end{array}$ & $\begin{array}{l}\text { 2. Pate } \\
\text { July } 1997 \\
\text { RL-TP13 }\end{array}$ & $\begin{array}{c}\text { 4. Dict. Rev. } \\
0\end{array}$ \\
\hline $\begin{array}{l}\text { 5. Contract WBS No. } \\
\text { 1.5.1.1.1.8 }\end{array}$ & $\begin{array}{l}\text { 6. Corresponding } \\
\text { FDS No. }\end{array}$ & $\begin{array}{l}\text { 7. Baseline CR No. } \\
\text { 8. Organization Name } \\
\text { DynCorp Operations }\end{array}$ & $\begin{array}{l}\text { 9. B \& R No. } \\
\text { EW7070201 }\end{array}$ \\
\hline
\end{tabular}

10. Scope of Work

This workscope would include projects related to reducing site mortgages by replacing telecommunications equipment that is antiquated and expensive to maintain. Where possible, this equipment would be replaced by energy saving equipment, thereby saving the entire site incremental costs. Also, this activity would include removal of legacy equipment and cable, which would be sold for salvage value.

Currently, there are no planned projects in FY 1998. 


\section{HANFORD SITE WORK BREAKDOWN STRUCTURE DICTIONARY} LANDLORD

WBS: 1.5

\begin{tabular}{|l|l|l|l|}
\hline $\begin{array}{l}\text { 1. Activity Title: } \\
\text { Infrastructure Mortgage Reduction - } \\
\text { Water }\end{array}$ & $\begin{array}{l}\text { 2Date } \\
\text { July } 1997\end{array}$ & $\begin{array}{l}\text { 3. PBS Number } \\
\text { RL-TP13 }\end{array}$ & $\begin{array}{c}\text { 4. Dict. Rev. } \\
0\end{array}$ \\
\hline 5. Contract WBS No. & $\begin{array}{l}\text { 6. Corresponding } \\
\text { FDS No. }\end{array}$ & $\begin{array}{l}\text { 7. Baseline CR No. } \\
\text { 1.5.1.1.1.9 }\end{array}$ & \\
\hline $\begin{array}{l}\text { 8. Organization Name } \\
\text { DynCorp Operations }\end{array}$ & $\begin{array}{l}\text { 9. B \& R No. } \\
\text { EW7070201 }\end{array}$ \\
\hline
\end{tabular}

10. Scope of Work

This workscope would include projects related to reducing site mortgages by replacing water equipment and components that are antiquated and expensive to maintain. Where possible, this equipment would be replaced by energy saving equipment, thereby saving the entire site incremental costs.

Currently, there are no planned projects in FY 1998. 


\section{LANDLORD PROJECT \\ WBS 1.5}

FY 1998 Work Plan

HNF-SP-1242, Rev. 0

\section{HANFORD SITE WORK BREAKOOWN STRUCTURE DICTIONARY \\ LANDLORD}

WBS: 1.5

\begin{tabular}{|l|l|l|l|}
\hline $\begin{array}{l}\text { 1. Activity Title: } \\
\begin{array}{l}\text { Core Infrastructure Maintenance - } \\
\text { Administration }\end{array}\end{array}$ & $\begin{array}{l}\text { 2Date } \\
\text { July } 1997\end{array}$ & $\begin{array}{l}\text { 3. PBS Number } \\
\text { RL-TP13 }\end{array}$ & 4. Dict. Rev. \\
0 \\
\hline $\begin{array}{l}\text { 5. Contract WBS No. } \\
\text { 1.5.1.1.2.1 }\end{array}$ & $\begin{array}{l}\text { 6. Corresponding } \\
\text { FDS No. }\end{array}$ & $\begin{array}{l}\text { 7. Baseline CR No. } \\
\text { 8. Organization Name }\end{array}$ & 9. B \& R No. \\
DynCorp Operations & EW7070201 \\
\hline
\end{tabular}

10. Scope of Work

Support entire Landlord Program in planning and reporting efforts throughout the fiscal year. Also supports Iand Use Planning and Asset Conversion activities. 


\section{LANDLORD PROJECT \\ WBS 1.5}

FY 1998 Work Plan

HNF-SP-1242, Rev. 0

\section{HANFORD SITE WORK BREAKDOWN STRUCTURE DICTIONARY LANDLORD}

WBS: 1.5

\begin{tabular}{|l|l|l|c|}
\hline $\begin{array}{l}\text { 1. Activity Title: } \\
\begin{array}{l}\text { Core Infrastructure Maintenance - } \\
\text { Program Management }\end{array}\end{array}$ & $\begin{array}{l}\text { 2Date } \\
\text { July } 1997 \\
\text { RL-TP13 }\end{array}$ & $\begin{array}{c}\text { 4. Dict. Rev. } \\
0\end{array}$ \\
\hline $\begin{array}{l}\text { 5. Contract WBS No. } \\
\text { 1.5.1.1.2.1A }\end{array}$ & $\begin{array}{l}\text { 6. Corresponding } \\
\text { FDS No. }\end{array}$ & $\begin{array}{l}\text { 7. Baseline CR No. } \\
\text { 8. Organization Name }\end{array}$ & $\begin{array}{l}\text { 9. B \& R No. } \\
\text { DynCorp Operations }\end{array}$ \\
\hline
\end{tabular}

10. Scope of Work

This WBS of the Hanford Landlord Program provides program integration activities for the Landlord Program and in support of the Hanford Site. Funding is required to ensure the site wide infrastructure is adequately managed. To safely fulfill the present and future mission requirements at the Hanford Site, a sound basic infrastructure to maintain essential services is necessary. Infrastructure is defined as "the basic facilities, equipment, and installations needed in order for the systems/operations of the Hanford Site to properly function". 


\section{HANFORD SITE WORK BREAKDOWN STRUCTURE DICTIONARY LANDLORD}

WBS: 1.5

\begin{tabular}{|l|l|l|l|}
\hline $\begin{array}{l}\text { 1. Activity Title: } \\
\begin{array}{l}\text { Core Inf. Maint. - Land Use/ Planning/ } \\
\text { Asset Conversion/Mapping Services }\end{array}\end{array}$ & $\begin{array}{l}\text { 2Date } \\
\text { July } 1997 \\
\text { RL-TP13 }\end{array}$ & $\begin{array}{c}\text { 4. Dict. Rev. } \\
0\end{array}$ \\
\hline \begin{tabular}{l|l|} 
5. Contract WBS No. \\
1.5.1.1.1B
\end{tabular} & $\begin{array}{l}\text { 6. Corresponding } \\
\text { FDS No. }\end{array}$ & 7. Baseline CR No. \\
\hline $\begin{array}{l}\text { 8. Organization Name } \\
\text { DynCorp Operations }\end{array}$ & $\begin{array}{l}\text { 9. B \& R No. } \\
\text { EW7070201 }\end{array}$ \\
\hline
\end{tabular}

10. Scope of Work

Specific activities of the Land Use Planning/Asset Conversion function include: a) site selection for individual construction projects; b) site plans for major complexes and end-state land use plans within each operating area at Hanford; c) comprehensive land use planning for the overall site including integration with other site wide plans such as environmental, strategic, systems engineering, environmental, biological, ecologic/cultural, infrastructure, demolition, economic development/transition/asset conversion, site restoration, facility use/upgrades, safety, security, coordination of construction schedules, excavation permit approval, etc. c activities of the Land Use Planning/Asset Conversion function include: a) site selection for individual construction projects; b) site plans for major complexes and end-state land use plans within each operating area at Hanford; c) comprehensive land use planning for the overall site including integration with other site wide plans such as environmental, strategic, systems engineering, environmental, biological, ecologic/cultural, infrastructure, demolition, economic development/transition/asset conversion, site restoration, facility use/upgrades, safety, security, coordination of construction schedules, excavation permit approval, etc. Activities for mapping services include the base system maintenance and operations. 


\section{LANDLORD PROJECT}

WBS 1.5

FY 1998 Work Plan

HNF-SP-1242, Rev. 0

\section{HANFORD SITE WORK BREAKDOWN STRUCTURE DICTIONARY LANDLORD}

WBS: 1.5

\begin{tabular}{|l|l|l|l|}
\hline $\begin{array}{l}\text { 1. Activity Title: } \\
\text { Core Infrastructure Maintenance- } \\
\text { Business Systems }\end{array}$ & $\begin{array}{l}\text { 2 Date } \\
\text { July } 1997\end{array}$ & $\begin{array}{l}\text { 3. PBS Number } \\
\text { RL-TP13 }\end{array}$ & $\begin{array}{c}\text { 4. Dict. Rev. } \\
0\end{array}$ \\
\hline $\begin{array}{l}\text { 5. Contract WBS No. } \\
\text { 1.5.1.1.2.2 }\end{array}$ & $\begin{array}{l}\text { 6. Corresponding } \\
\text { FDS No. }\end{array}$ & 7. Baseline CR No. \\
\hline $\begin{array}{l}\text { 8. Organization Name } \\
\text { DynCorp Operations }\end{array}$ & $\begin{array}{l}\text { 9. B \& R No. } \\
\text { EW7070201 }\end{array}$ \\
\hline
\end{tabular}

10. Scope of Work

No workscope is currentiy planned in FY 1998 or 1999. 


\section{LANDLORD PROJECT \\ WBS 1.5}

FY 1998 Work Plan

HNF-SP-1242, Rev. 0

\section{HANFORD SITE WORK BREAKDOWN STRUCTURE DICTIONARY \\ LANDLORD}

WBS: 1.5

\begin{tabular}{|c|c|c|c|}
\hline $\begin{array}{l}\text { 1. Activity Title: } \\
\text { Core Infrastructure Maintenance - } \\
\text { Sewer }\end{array}$ & $\begin{array}{l}2 \text { Date } \\
\text { July } 1997 .\end{array}$ & $\begin{array}{l}\text { 3. PBS Number } \\
\text { RL-TP13 }\end{array}$ & $\begin{array}{l}\text { 4. Dict. Rev. } \\
0\end{array}$ \\
\hline $\begin{array}{l}\text { 5. Contract WBS No. } \\
1.5 .1 .1 .2 .3\end{array}$ & $\begin{array}{l}\text { 6. Corresponding } \\
\text { FDS No. }\end{array}$ & \multicolumn{2}{|c|}{ 7. Baseline CR No. } \\
\hline $\begin{array}{l}\text { 8. Organization Name } \\
\text { DynCorp Operations }\end{array}$ & \multicolumn{3}{|l|}{$\begin{array}{l}\text { 9. B \& R No. } \\
\text { EW7070201 }\end{array}$} \\
\hline \multicolumn{4}{|c|}{$\begin{array}{l}\text { 10. Scope of Work } \\
\text { This activity replaces failing sanitary sewer systems. These sanitary sewer systems are beyond their useful life and } \\
\text { do not meet current regulatory requirements. The replacements will be made according to WHC 246-272 and the } \\
\text { priorities set in WHC-SD-LL-SP-001, "200/600 Area Sanitary Waste Water Master Plan," March 1996. }\end{array}$} \\
\hline
\end{tabular}




\section{LANDLORD PROJECT \\ WBS 1.5}

FY 1998 Work Plan

HNF-SP-1242, Rev. 0

\section{HANFORD SITE WORK BREAKDOWN STRUCTURE DICTIONARY \\ LANDLORD}

WBS: 1.5

\begin{tabular}{|c|c|c|c|c|}
\hline \multicolumn{2}{|c|}{$\begin{array}{l}\text { 1. Activity Title: } \\
\text { Core Infrastructure Maintenance - Final Regulatory Disposition of } \\
300 \text { Area and Outer Area Abandoned Septic Systems }\end{array}$} & $\begin{array}{l}2 \text { Date } \\
\text { July } 1997\end{array}$ & $\begin{array}{l}\text { 3. PBS Number } \\
\text { RL-TP13 }\end{array}$ & $\begin{array}{c}\text { 4. Dict. Rev. } \\
0\end{array}$ \\
\hline $\begin{array}{l}\text { 5. Contract WBS No. } \\
\text { 1.5.1.1.2.3A }\end{array}$ & . & $\begin{array}{l}\text { 6. Corres. } \\
\text { FDS No. }\end{array}$ & \multicolumn{2}{|c|}{ 7. Baseline CR No. } \\
\hline $\begin{array}{l}\text { 8. Organization Name } \\
\text { DynCorp Operations }\end{array}$ & \multicolumn{4}{|l|}{$\begin{array}{l}\text { 9. B \& RNo. } \\
\text { EW7070201 }\end{array}$} \\
\hline
\end{tabular}

10. Scope of Work

To meet Washington Administrative codes, shutdown systems must be eventually put in proper regulatory disposition. This funding will specifically disposition the 300 Area septic system that was shutdown in 1996 when 300 Area sewage began discharging to the City of Richland sanitary sewer system as well as miscellaneous systems in the outer areas. 


\section{LANDLORD PROJECT \\ WBS 1.5}

FY 1998 Work Plan

HNF-SP-1242, Rev. 0

\section{HANFORD SITE WORK BREAKDOWN STRUCTURE DICTIONARY}

\section{LANDLORD}

WBS: 1.5

\begin{tabular}{|c|c|c|c|}
\hline $\begin{array}{l}\text { 1. Activity Title: } \\
\text { Core Infrastructure Maintenance - } \\
\text { Project L-272 }\end{array}$ & $\begin{array}{l}\text { 2 Date } \\
\text { July } 1997\end{array}$ & $\begin{array}{l}\text { 3. PBS Number } \\
\text { RL-TP13 }\end{array}$ & $\begin{array}{c}\text { 4. Dict. Rev. } \\
0\end{array}$ \\
\hline $\begin{array}{l}\text { 5. Contract WBS No. } \\
\text { 1.5.1.1.2.3E }\end{array}$ & $\begin{array}{l}\text { 6. Corresponding } \\
\text { FDS No. }\end{array}$ & 7. Baseline CR No. & \\
\hline $\begin{array}{l}\text { 8. Organization Name } \\
\text { DynCorp Operations }\end{array}$ & $\begin{array}{l}\text { 9. B \& R No. } \\
\text { EW7070201 }\end{array}$ & & \\
\hline \multicolumn{4}{|l|}{ 10. Scope of Work } \\
\hline \multicolumn{4}{|c|}{$\begin{array}{l}\text { During FY 1997, this activity began the replacement of the septic systems in the central core of } 200 \text { East Area which } \\
\text { have failed or are failing and do not meet current regulatory requirements. These systems are beyond their useful life } \\
\text { and are required to support the } 200 \text { Area cleanup and waste management activities. The replacement will be made } \\
\text { according to WAC } 246-272 \text { and the priorities set in WHC-SD-LL-SP-001, "200/600 Area Sanitary Waste Water } \\
\text { Master Plan," March } 1996 \text {. }\end{array}$} \\
\hline
\end{tabular}
according to WAC 246-272 and the priorities set in WHC-SD-LL-SP-001, "200/600 Area Sanitary Waste Water Master Plan," March 1996. 


\section{LANDLORD PROJECT \\ WBS 1.5}

FY 1998 Work Plan

HNF-SP-1242, Rev. 0

\section{HANFORD SITE WORK BREAKDOWN STRUCTURE DICTIONARY \\ LANDLORD}

WBS: 1.5

\begin{tabular}{|c|c|c|c|}
\hline $\begin{array}{l}\text { 1. Activity Title: } \\
\text { Core Infrastructure Maintenance - } \\
\text { Roads/Rail/Heavy Equipment }\end{array}$ & $\begin{array}{l}2 \text { Date } \\
\text { July } 1997\end{array}$ & $\begin{array}{l}\text { 3. PBS Number } \\
\text { RL-TP13 }\end{array}$ & $\begin{array}{c}\text { 4. Dict. Rev. } \\
0\end{array}$ \\
\hline $\begin{array}{l}\text { 5. Contract WBS No. } \\
\text { 1.5.1.1.2.4 }\end{array}$ & $\begin{array}{l}\text { 6. Corresponding } \\
\text { FDS No. }\end{array}$ & \multicolumn{2}{|l|}{ 7. Baseline CRNo. } \\
\hline $\begin{array}{l}\text { 8. Organization Name } \\
\text { DynCorp Operations }\end{array}$ & \multicolumn{3}{|l|}{$\begin{array}{l}\text { 9. B \& R No. } \\
\text { EW7070201 }\end{array}$} \\
\hline \multicolumn{4}{|c|}{$\begin{array}{l}\text { 10. Scope of Work } \\
\text { This workscope includes the overlay or chip seal of existing road surfaces, as well as replacement of essential } \\
\text { railroad and heavy equipment on the Hanford Site. These are common access roads and general rail and equipment } \\
\text { not particularly related to any one program. Completing the activities associated with this scope extends the life } \\
\text { cycle of the current essential infrastructure systems significantly reducing the possibility of complete overhauls and } \\
\text { replacements at a much greater cost. }\end{array}$} \\
\hline
\end{tabular}




\section{LANDLORD PROJECT \\ WBS 1.5}

\section{HANFORD SITE WORK BREAKDOWN STRUCTURE DICTIONARY \\ LANDLORD}

WBS: 1.5

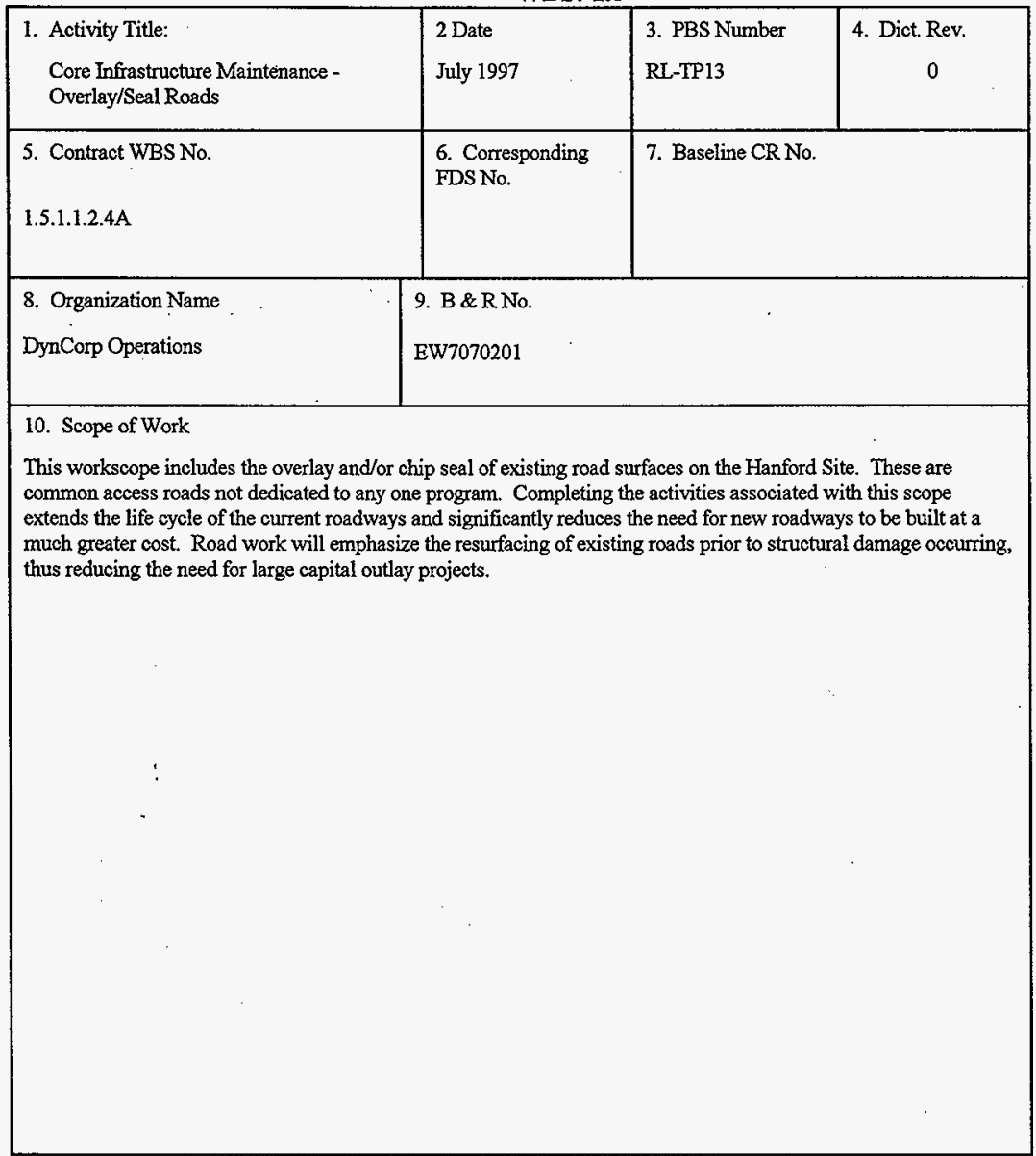




\section{HANFORD SITE WORK BREAKDOWN STRUCTURE DICTIONARY}

\section{LANDLORD}

WBS: 1.5

\begin{tabular}{|l|l|l|l|}
\hline $\begin{array}{l}\text { 1. Activity Title: } \\
\begin{array}{l}\text { Core Infrastructure Maintenance - } \\
\text { Electrical Distribution }\end{array}\end{array}$ & $\begin{array}{l}\text { 2Date } \\
\text { July } 1997\end{array}$ & $\begin{array}{c}\text { 4. Dict. Rev. } \\
\text { RL-TPI3 }\end{array}$ \\
\hline 5. Contract WBS No. & $\begin{array}{l}\text { 6. Corresponding } \\
\text { FDS No. }\end{array}$ & 7. Baseline CR No. \\
$\begin{array}{l}\text { 1.5.1.1.2.5 } \\
\text { 8. Organization Name }\end{array}$ & 9. B \& R No. \\
DynCorp Operations & EW7070201 & \\
\hline
\end{tabular}

10. Scope of Work

This workscope will provide essential repairs, replacements, and/or upgrades to the site electrical distribution system. 


\section{LANDLORD PROJECT \\ WBS 1.5}

FY 1998 Work Plan

HNF-SP-1242, Rev. 0

AANFORD SITE WORK BREAKDOWN STRUCTURE DICTIONARY

LANDLORD

WBS: 1.5

\begin{tabular}{|l|l|l|l|}
\hline $\begin{array}{l}\text { 1. Activity Title: } \\
\begin{array}{l}\text { Core Infrastructure Maintenance - } \\
\text { Emergency Facilities/Services }\end{array}\end{array}$ & $\begin{array}{l}\text { 2Date } \\
\text { July } 1997\end{array}$ & $\begin{array}{l}\text { 4. PBS Number } \\
\text { RL-TP13 }\end{array}$ & 0 \\
\hline $\begin{array}{l}\text { 5. Contract WBS No. } \\
1.5 .1 .1 .2 .6\end{array}$ & $\begin{array}{l}\text { 6. Corresponding } \\
\text { FDS No. } \\
\text { 8. Organization Name }\end{array}$ & $\begin{array}{l}\text { 7. Baseline CR No. } \\
\text { DynCorp Operations }\end{array}$ & $\begin{array}{l}\text { 9. B \& R No. } \\
\text { EW7070201 }\end{array}$ \\
\hline
\end{tabular}

10. Scope of Work

This activity provides for essential equipment and facilities for emergency services. 


\section{LANDLORD PROJECT \\ WBS 1.5}

\section{HANFORD SITE WORK BREAKDOWN STRUCTURE DICTIONARY LANDLORD}

WBS: 1.5

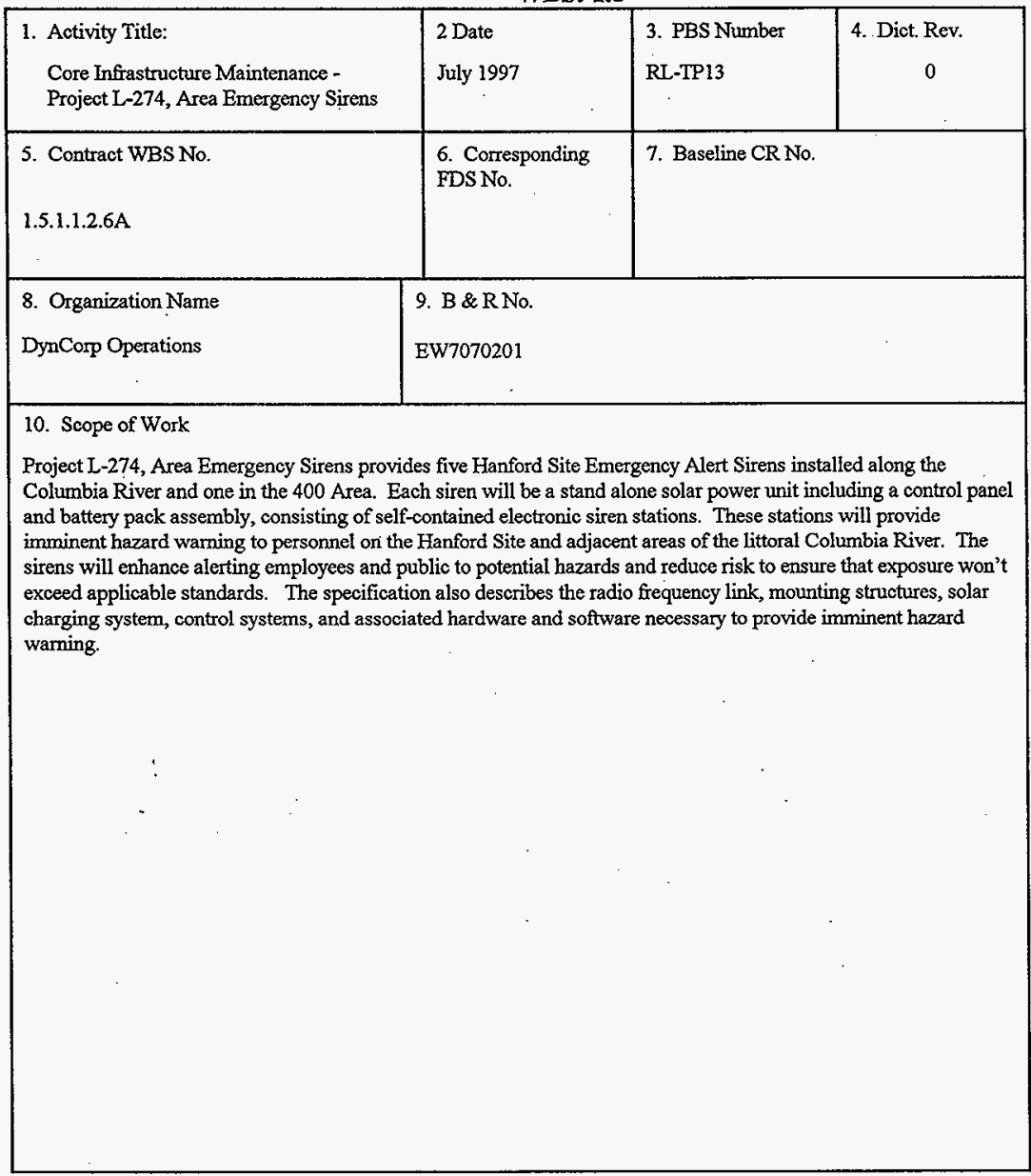




\section{LANDLORD PROJECT \\ WBS 1.5}

FY 1998 Work Plan

HNF-SP-1242, Rev. 0

\section{HANFORD SITE WORK BREAKDOWN STRUCTURE DICTIONARY \\ LANDLORD}

WBS: 1.5

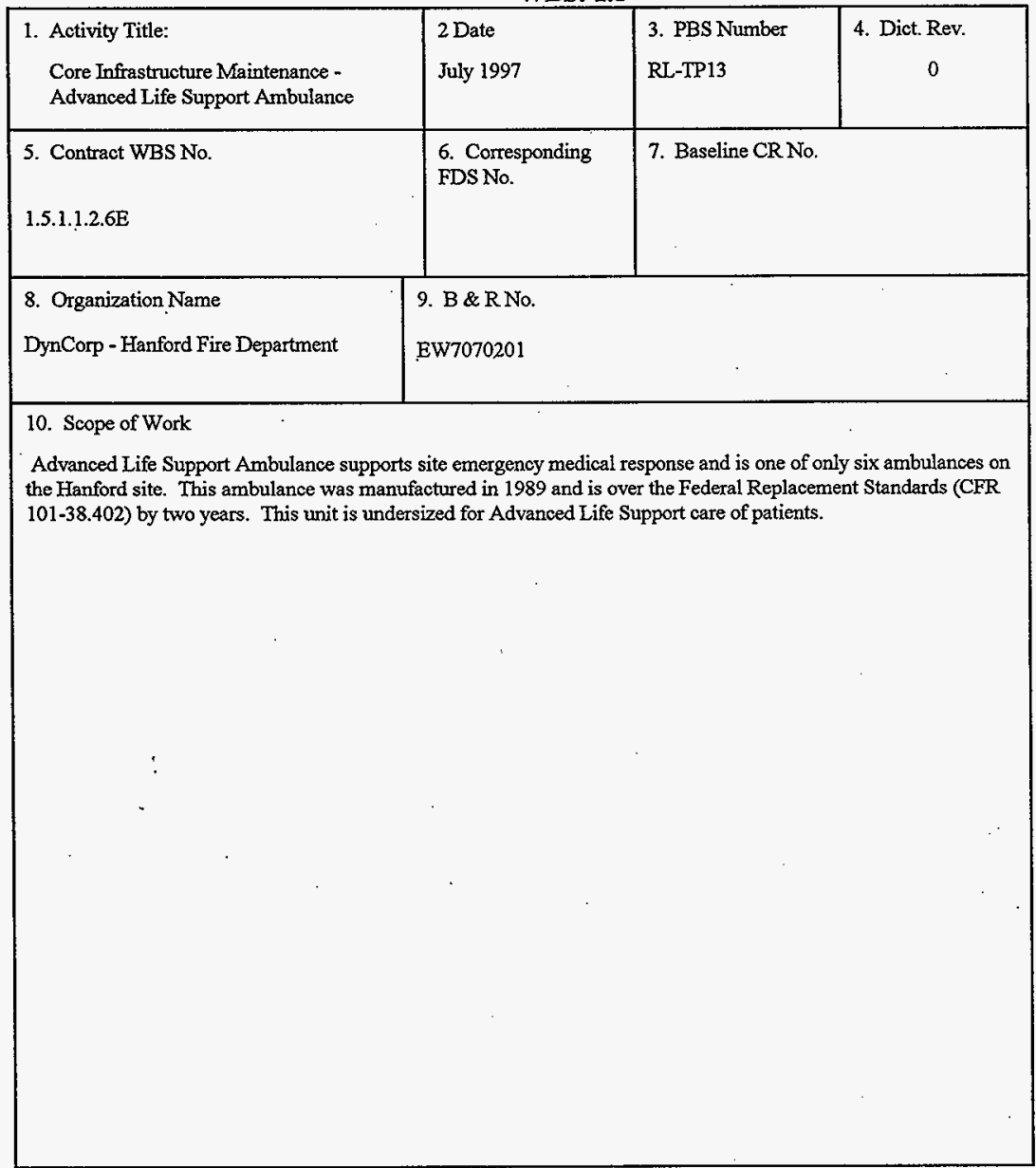




\section{LANDLORD PROJECT \\ WBS 1.5}

\section{HANFORD SITE WORK BREAKDOWN STRUCTURE DICTIONARY LANDLORD}

WBS: 1.5

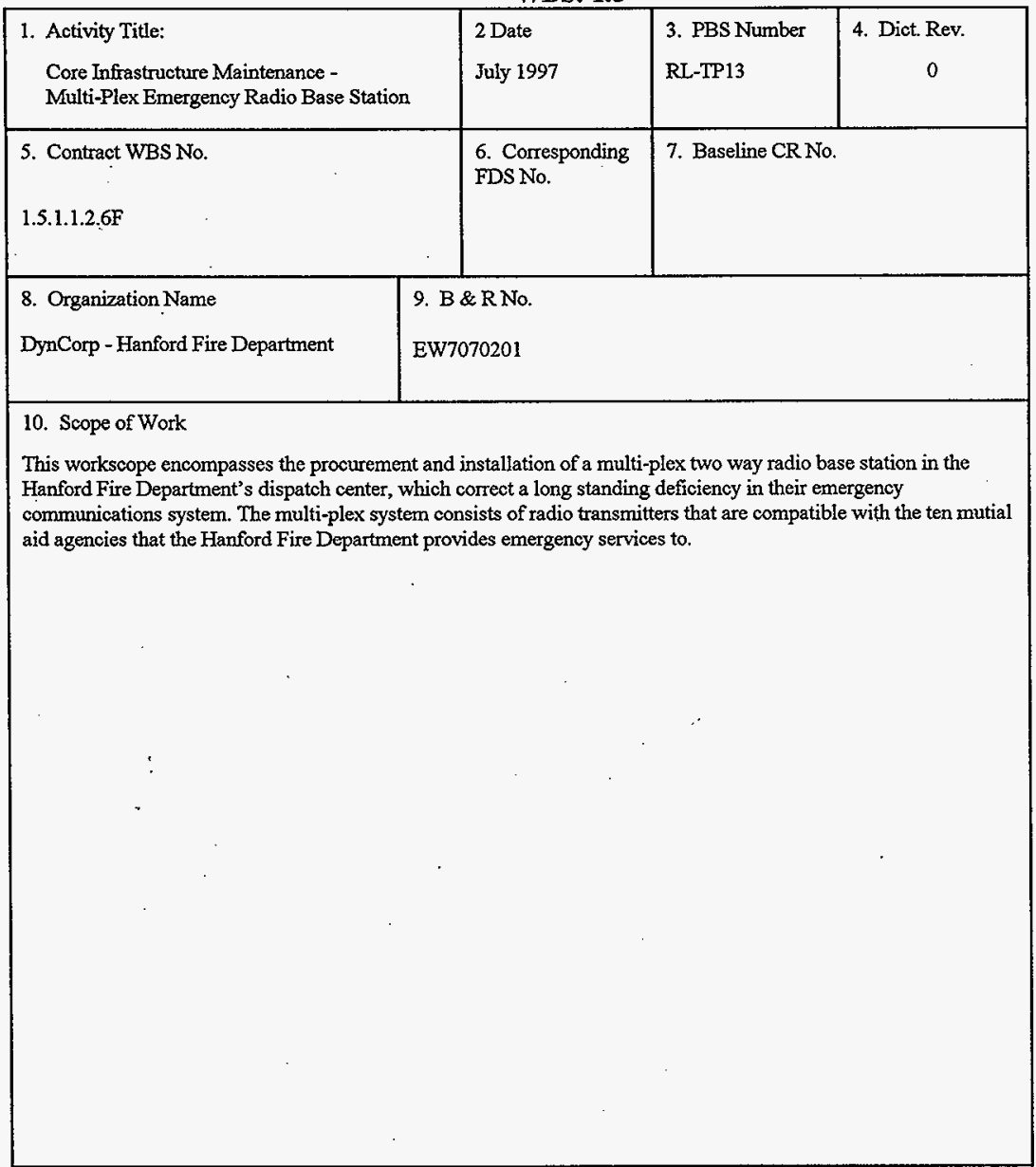




\section{LANDLORD PROJECT \\ WBS 1.5}

FY 1998 Work Plan

HNF-SP-1242, Rev. 0

\section{HANEORD SITE WORK BREAKDOWN STRUCTURE DICTIONARY \\ LANDLORD}

WBS: 1.5

\begin{tabular}{|l|l|l|c|}
\hline $\begin{array}{l}\text { 1. Activity Title: } \\
\begin{array}{l}\text { Core Infrastructure Maintenance - } \\
\text { Fire Engine Acrial Ladder (50) }\end{array}\end{array}$ & $\begin{array}{l}\text { 3. Pate } \\
\text { July } 1997 \\
\text { RL-TP13 }\end{array}$ & $\begin{array}{c}\text { 4. Dict. Rev. } \\
0\end{array}$ \\
\hline $\begin{array}{l}\text { 5. Contract WBS No. } \\
\text { 1.5.1.1.2.6G }\end{array}$ & $\begin{array}{l}\text { 6. Corresponding } \\
\text { FDS No. }\end{array}$ & $\begin{array}{l}\text { 7. Baseline CR No. } \\
\text { 8. Organization Name } \\
\text { DynCorp - Hanford Fire Department }\end{array}$ & $\begin{array}{l}\text { 9. B \& RNo. } \\
\text { EW7070201 }\end{array}$ \\
\hline
\end{tabular}

10. Scope of Work

This activity replaces a 1978 fure engine which is one of only four aerial fire engines on the Hanford Site. This piece of equipment should have been replaced eleven years ago according to the federal replacement standard CFR 101-38.402. 
HANFORD SITE WORK BREAKDOWN STRUCTURE DICTIONARY

LANDLORD

WBS: 1.5

\begin{tabular}{|l|l|l|l|}
\hline $\begin{array}{l}\text { 1. Activity Title: } \\
\text { Core Infrastructure Maintenance - } \\
\text { Project L-275 }\end{array}$ & $\begin{array}{l}\text { 2 Date } \\
\text { July } 1997\end{array}$ & $\begin{array}{l}\text { 3. PBS Number } \\
\text { RL-TP13 }\end{array}$ & $\begin{array}{c}\text { 4. Dict. Rev. } \\
0\end{array}$ \\
\hline 5. Contract WBS No. & $\begin{array}{l}\text { 6. Corresponding } \\
\text { FDS No. }\end{array}$ & $\begin{array}{l}\text { 7. Baseline CR No. } \\
\text { 1.5.1.1.2.6H }\end{array}$ & $\begin{array}{l}\text { 9. B \& R No. } \\
\text { EW7070201 }\end{array}$ \\
\hline $\begin{array}{l}\text { 8. Organization Name } \\
\text { DynCorp - Hanford Fire Department }\end{array}$ & \\
\hline
\end{tabular}

10. Scope of Work

This activity relocates an existing modular office complex to the east of the 200 Area fire station to allow consolidation of the Hanford Site Emergency Services Administration and Training function. This allows the renovation of the existing over crowded and deteriorated 200 Area fire station in FY 1999. 


\section{HANFORD SITE WORK BREAKDOWN STRUCTURE DICTIONARY LANDLORD}

WBS: 1.5

\begin{tabular}{|l|l|l|c|}
\hline $\begin{array}{l}\text { 1. Activity Title: } \\
\begin{array}{l}\text { Core Infrastructure Maintenance - } \\
\text { Facilities/Shops/PNNL Laboratories }\end{array}\end{array}$ & $\begin{array}{l}\text { 2Date } \\
\text { July } 1997\end{array}$ & $\begin{array}{l}\text { 3. PBS Number } \\
\text { RL-TP13 }\end{array}$ & 0 \\
\hline 5. Contract WBS No. & $\begin{array}{l}\text { 6. Corresponding } \\
\text { FDS No. }\end{array}$ & 7. Baseline CR No. \\
1.5.1.1.2.7 & 9. B \& R No. \\
\hline $\begin{array}{l}\text { 8. Organization Name } \\
\text { DynCorp Operations }\end{array}$ & EW7070201 & \\
\hline
\end{tabular}

10. Scope of Work

This workscope includes mortgage reduction activities to reduce the number of occupied general purpose facilities on the Hanford Site. This includes isolating the facilities from all utilities in a cost cutting effort, thereby reducing the necessary surveillance costs. In cases where the fire protection systems must be maintained after the facility is vacated, the utility costs are included in this activity. The buildings can then be transitioned to the demolition program or can be redeployed to private entities. 


\section{HANFORD SITE WORK BREAKDOWN STRUCTURE DICTIONARY LANDLORD}

WBS: 1.5

\begin{tabular}{|c|c|c|c|}
\hline $\begin{array}{l}\text { 1. Activity Title: } \\
\text { Core Infrastructure Maintenance - } \\
\text { Sanitary Solid Waste Landfill Eng. Study }\end{array}$ & $\begin{array}{l}2 \text { Date } \\
\text { July } 1997\end{array}$ & $\begin{array}{l}\text { 3. PBS Number } \\
\text { RL-IP13 }\end{array}$ & $\begin{array}{c}\text { 4. Dict. Rev. } \\
0\end{array}$ \\
\hline $\begin{array}{l}\text { 5. Contract WBS No. } \\
1.5 \cdot 1.1 .2 .7 \mathrm{~A}\end{array}$ & $\begin{array}{l}\text { 6. Corresponding } \\
\text { FDS No. }\end{array}$ & \multicolumn{2}{|c|}{ 7. Baseline CR No. } \\
\hline $\begin{array}{l}\text { 8. Organization Name } \\
\text { DynCorp Operations }\end{array}$ & \multicolumn{3}{|l|}{$\begin{array}{l}\text { 9. B \& RNo. } \\
\text { EW7070201 }\end{array}$} \\
\hline \multicolumn{4}{|c|}{$\begin{array}{l}\text { 10. Scope of Work } \\
\text { Provides necessary preliminary engineering to support a FY } 2001 \text { line item project. The sanitary landfill was closed } \\
\text { during } 1996 \text {. Closure of a landfill requires a closure plan be implemented within } 180 \text { days (Washington } \\
\text { Administrative Code and Environmental Protection Agency Act). An interim closure plan was developed in late FY } \\
\text { 1996, however the Washington State Department of Ecology disapproved it due to the amount of water discovered in } \\
\text { the landfill monitoring wells. }\end{array}$} \\
\hline
\end{tabular}




\section{HANFORD SITE WORK BREAKDOWN STRUCTURE DICTIONARY LANDLORD}

WBS: 1.5

\begin{tabular}{|l|l|l|c|}
\hline $\begin{array}{l}\text { 1. Activity Title: } \\
\begin{array}{c}\text { Core Infrastructure Maintenance - PNNL } \\
\text { Computer Repl. for Radiological Assessments }\end{array}\end{array}$ & $\begin{array}{l}\text { 2Date } \\
\text { July } 1997\end{array}$ & $\begin{array}{l}\text { 3. PBS Number } \\
\text { RL-TP13 }\end{array}$ & $\begin{array}{c}\text { 4. Dict. Rev. } \\
0\end{array}$ \\
\hline $\begin{array}{l}\text { 5. Contract WBS No. } \\
\text { 1.5.1.1.2.7B }\end{array}$ & $\begin{array}{l}\text { 6. Corresponding } \\
\text { FDS No. }\end{array}$ & $\begin{array}{l}\text { 7. Baseline CR No. } \\
\text { 8. Organization Name }\end{array}$ & 9. B \& R No. \\
PNNL & EW7070201. \\
\hline
\end{tabular}

\section{Scope of Work}

This replaces a 10 year old system and is essential for generating the data used to estimate the amounts of radioactive material in the Hanford workers as part of the overall program to demonstrate compliance with the regulatory exposure limits in $10 \mathrm{CFR} 835$. Recent system failures have resulted in the measurement data for up to five persons being completely lost. The current system is not economically repairable. 


\section{LANDLORD PROJECT \\ WBS 1.5}

FY 1998 Work Plan

HNF-SP-1242, Rev. 0

\section{HANFORD SITE WORK BREAKDOWN STRUCTURE DICTIONARY \\ LANDLORD}

WBS: 1.5

\begin{tabular}{|c|c|c|c|}
\hline $\begin{array}{l}\text { 1. Activity Title: } \\
\text { Core Infrastructure Maintenance - } \\
\text { Telecommunications }\end{array}$ & $\begin{array}{l}2 \text { Date } \\
\text { July } 1997\end{array}$ & $\begin{array}{l}\text { 3. PBS Number } \\
\text { RL-TP13 }\end{array}$ & $\begin{array}{c}\text { 4. Dict. Rev. } \\
0\end{array}$ \\
\hline $\begin{array}{l}\text { 5. Contract WBS No. } \\
\text { 1.5.1.1.2.8 }\end{array}$ & $\begin{array}{l}\text { 6. Corresponding } \\
\text { FDS No. }\end{array}$ & \multicolumn{2}{|l|}{ 7. Baseline CRNo. } \\
\hline $\begin{array}{l}\text { 8. Organization Name } \\
\text { DynCorp Operations }\end{array}$ & \multicolumn{3}{|l|}{$\begin{array}{l}\text { 9. B \& RNo. } \\
\text { EW7070201 }\end{array}$} \\
\hline \multicolumn{4}{|c|}{$\begin{array}{l}\text { 10. Scope of Work } \\
\text { This workscope includes maintaining the current essential infrastructure related to the site telecommunications } \\
\text { systems. In FY } 1997 \text { and } 1998 \text { this activity installs a new fiber optic backbone for the Hanford Site } \\
\text { telecommunication system. This new system will replace the obsolete and failing Hanford Site microwave systems. } \\
\text { The fiber optic system is necessary to carry emergency signals, essential electrical distribution switching signals, and } \\
\text { other vital Hanford Site electronic data. The Hanford Site depends on the telecommunication system to carry on the } \\
\text { day-to-day activities related to the Environmental cleanup and waste management. }\end{array}$} \\
\hline
\end{tabular}




\section{HANFORD SITE WORK BREAKDOWN STRUCTURE DICTIONARY LANDLORD}

WBS: 1.5

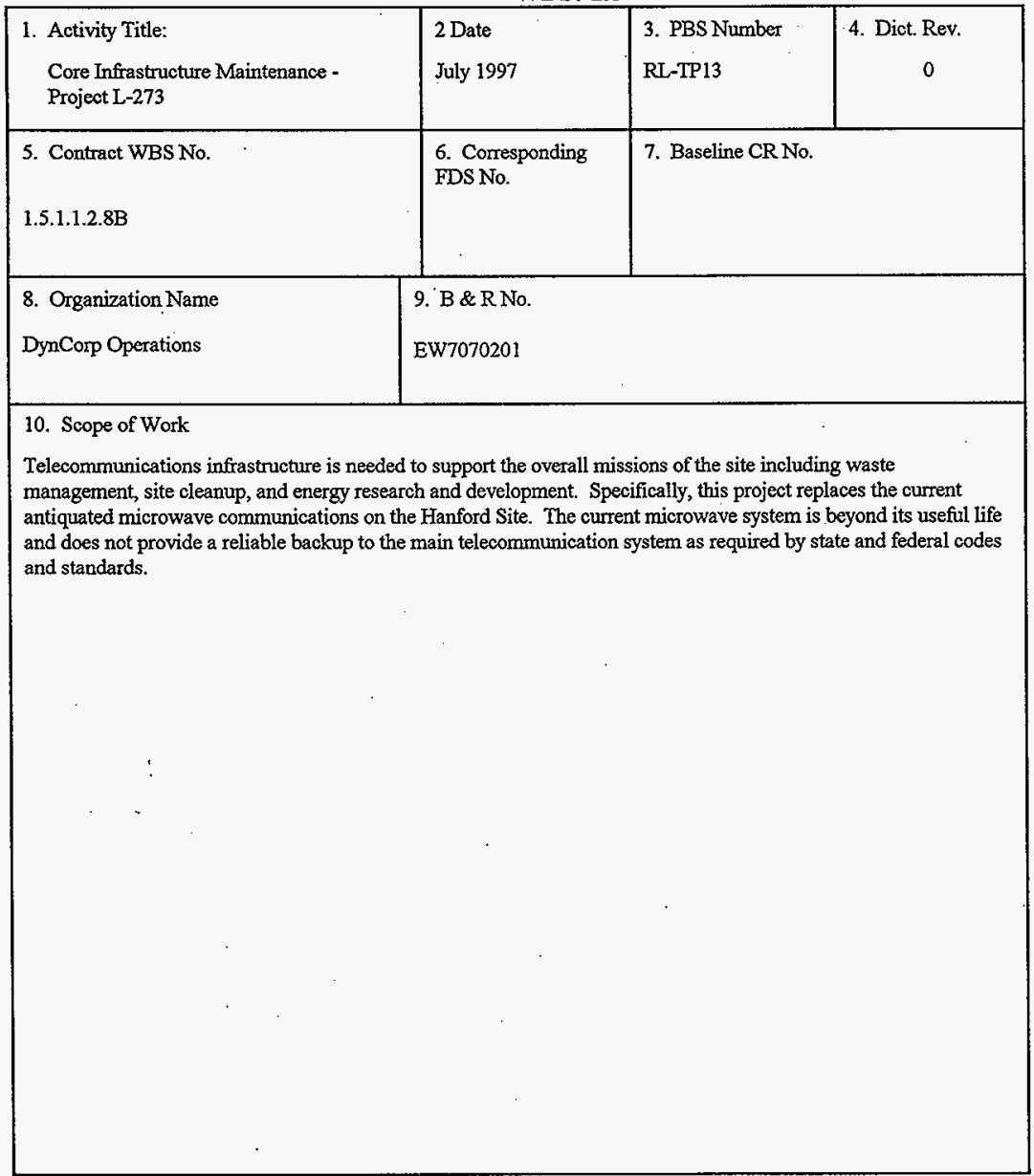




\section{LANDLORD PROJECT \\ WBS 1.5}

FY 1998 Work Plan

HNF-SP-1242, Rev. 0

\section{HANFORD SITE WORK BREAKDOWN STRUCTURE DICTIONARY \\ LANDLORD}

WBS: 1.5

\begin{tabular}{|l|l|l|l|}
\hline $\begin{array}{l}\text { 1. Activity Title: } \\
\begin{array}{l}\text { Core Infrastructure Maintenance - } \\
\text { Project L-279 }\end{array}\end{array}$ & $\begin{array}{l}\text { 2 Date } \\
\text { July } 1997 \\
\text { RL-TP13 }\end{array}$ & $\begin{array}{c}\text { 4. Dict. Rev. } \\
0\end{array}$ \\
\hline \begin{tabular}{l|l|} 
5. Contract WBS No. \\
1.5.1.1.2.8C
\end{tabular} & $\begin{array}{l}\text { 6. Corresponding } \\
\text { FDS No. }\end{array}$ & 7. Baseline CR No. \\
\hline $\begin{array}{l}\text { 8. Organization Name } \\
\text { DynCorp - Hanford Fire Department }\end{array}$ & $\begin{array}{l}\text { 9. B \& R No. } \\
\text { EW7070201 }\end{array}$ \\
\hline
\end{tabular}

10. Scope of Work

Telecommunications infrastructure is needed to support the overall missions of the site including waste management, site cleanup, and energy research and development. Specifically, this project replaces the current antiquated microwave communications on the Hanford Site. The current microwave system is beyond its useful life and does not provide a reliable backup to the main telecommunication system as required by state and federal codes and standards. This project connects vital telecommunication systems for the outer area (100 and 200 Areas). 


\section{LANDLORD PROJECT \\ WBS 1.5}

FY 1998 Work Plan

HNF-SP-1242, Rev, 0

\section{HANFORD SITE WORK BREAKDOWN STRUCTURE DICTIONARY LANDLORD}

WBS: 1.5

\begin{tabular}{|c|c|c|c|}
\hline $\begin{array}{l}\text { 1. Activity Title: } \\
\text { Core Infrastructure Maintenance - } \\
\text { UPS for } 3719 \text { Building }\end{array}$ & $\begin{array}{l}2 \text { Date } \\
\text { July } 1997\end{array}$ & $\begin{array}{l}\text { 3. PBS Number } \\
\text { RL-TP13 }\end{array}$ & $\begin{array}{c}\text { 4. Dict. Rev. } \\
0\end{array}$ \\
\hline $\begin{array}{l}\text { 5. Contract WBS No. } \\
\text { 1.5.1.1.2.8D }\end{array}$ & $\begin{array}{l}\text { 6. Corresponding } \\
\text { FDS No. }\end{array}$ & \multicolumn{2}{|c|}{ 7. Baseline CR No. } \\
\hline $\begin{array}{l}\text { 8. Organization Name } \\
\text { DynCorp Operations }\end{array}$ & \multicolumn{3}{|l|}{$\begin{array}{l}\text { 9. B \& RNo. } \\
\text { EW7070201 }\end{array}$} \\
\hline \multicolumn{4}{|c|}{$\begin{array}{l}\text { 10. Scope of Work } \\
\text { This project upgrades the existing uninterruptable-power supply (UPS) system to the } 3719 \text { Building electrical } \\
\text { system. The UPS is a necessary backup system for telephone, HLAN, and priority telecommunications in the event } \\
\text { of an electrical outage. Loss of power to these telecommunication systems would eliminate vital telecommunication } \\
\text { links for emergency signals, data transmissions, and other Hanford Site related functions. }\end{array}$} \\
\hline
\end{tabular}




\section{LANDLORD PROJECT \\ WBS 1.5}

FY 1998 Work Plan

HNF-SP-1242, Rev, 0

\section{HANFORD SITE WORK BREAKDOWN STRUCTURE DICTIONARY LANDLORD}

WBS: 1.5

\begin{tabular}{|l|l|l|c|}
\hline $\begin{array}{l}\text { 1. Activity Title: } \\
\text { Core Infrastructure Maintenance - } \\
\text { Water }\end{array}$ & $\begin{array}{l}\text { 2Date } \\
\text { July } 1997\end{array}$ & $\begin{array}{l}\text { 3. PBS Number } \\
\text { RL-TP13 }\end{array}$ & $\begin{array}{c}\text { 4. Dict. Rev. } \\
0\end{array}$ \\
\hline $\begin{array}{l}\text { 5. Contract WBS No. } \\
1.5 .1 .1 .2 .9\end{array}$ & $\begin{array}{l}\text { 6. Corresponding } \\
\text { FDS No. }\end{array}$ & $\begin{array}{l}\text { 7. Baseline CR No. } \\
\text { 8. Organization Name } \\
\text { DynCorp Operations }\end{array}$ & $\begin{array}{l}\text { 9. B \& R No. } \\
\text { EW7070201 }\end{array}$ \\
\hline
\end{tabular}

10. Scope of Work

This workscope maintains and replaces essential site water system equipment and components. 


\section{HANFORD SITE WORK BREAKDOWN STRUCTURE DICTIONARY LANDLORD}

WBS: 1.5

\begin{tabular}{|c|c|c|c|}
\hline $\begin{array}{l}\text { 1. Activity Title: } \\
\text { Core Infrastructure Maintenance - } \\
\text { Project L-286 }\end{array}$ & $\begin{array}{l}\text { 2 Date } \\
\text { July } 1997\end{array}$ & $\begin{array}{l}\text { 3. PBS Number } \\
\text { RL-TP13 }\end{array}$ & $\begin{array}{c}\text { 4. Dict. Rev. } \\
0\end{array}$ \\
\hline $\begin{array}{l}\text { 5. Contract WBS No. } \\
\text { 1.5.1.1.2.9C/D }\end{array}$ & $\begin{array}{l}\text { 6. Corresponding } \\
\text { FDS No. }\end{array}$ & \multicolumn{2}{|c|}{ 7. Baseline CR No. } \\
\hline $\begin{array}{l}\text { 8. Organization Name } \\
\text { DynCorp Operations }\end{array}$ & \multicolumn{3}{|l|}{$\begin{array}{l}\text { 9. B \& RNo. } \\
\text { EW7070201 }\end{array}$} \\
\hline \multicolumn{4}{|c|}{$\begin{array}{l}\text { 10. Scope of Work } \\
\text { Installs booster pump(s) in interconnection line between the } 200 \text { East and } 200 \text { West Water System, the } 200 \text { East } \\
\text { Clearwell level and pump controls, the } 200 \text { East and } 200 \text { West Water Treatment Plants (WTPs) automated fire water } \\
\text { control system, } 200 \mathrm{E} \text { filters isolation and lay up, } 283 \mathrm{E} / \mathrm{W} \text { raw water inlet automated control, and remote } \\
\text { annunciation of the } 200 \text { Area WTPs. This project will eliminate the majority of water discharged to the unlined } \\
\text { trench while meeting domestic water and fire protection requirements for the outer area. }\end{array}$} \\
\hline
\end{tabular}




\section{LANDLORD PROJECT \\ WBS 1.5}

FY 1998 Work Plan

HNF-SP-1242, Rev. 0

\section{HANFORD SITE WORK BREAKDOWN STRUCTURE DICTIONARY LANDLORD}

WBS: 1.5

\begin{tabular}{|c|c|c|c|}
\hline $\begin{array}{l}\text { 1. Activity Title:- } \\
\text { Core Infrastructure Maintenance - } \\
\text { Project L-281 }\end{array}$ & $\begin{array}{l}2 \text { Date } \\
\text { July } 1997\end{array}$ & $\begin{array}{l}\text { 3. PBS Number } \\
\text { RL-TPI3 }\end{array}$ & $\begin{array}{c}\text { 4. Dict. Rev. } \\
0\end{array}$ \\
\hline $\begin{array}{l}\text { 5. Contract WBS No. } \\
\text { 1.5.1.1.2.9A/B. }\end{array}$ & $\begin{array}{l}\text { 6. Corresponding } \\
\text { FDS No. }\end{array}$ & \multicolumn{2}{|l|}{ 7. Baseline CR No. } \\
\hline $\begin{array}{l}\text { 8. Organization Name } \\
\text { DynCorp Operations }\end{array}$ & \multicolumn{3}{|l|}{$\begin{array}{l}\text { 9. B \& R No. } \\
\text { EW7070201 }\end{array}$} \\
\hline \multicolumn{4}{|c|}{$\begin{array}{l}\text { 10. Scope of Work } \\
\text { This project replaces the non-compliant and failing systems at the Plutonium Finishing Plant (PFP) and } 200 \text { West } \\
\text { Area Tank Farms Operations Facility (272-WA) in the central } 200 \text { West Area. These systems have required } \\
\text { accelerated pumping and additional maintenance to minimize sewage discharges to the ground. The DOE has made } \\
\text { commitments to Washington State Department of Ecology (WSDOE) to bring non-compliant septic systems into } \\
\text { compliance with state regulations (Master Plan WHC-SD-LL-SP-001, dated April 15, 1996). }\end{array}$} \\
\hline
\end{tabular}




\section{LANDLORD PROJECT \\ WBS 1.5}

FY 1998 Work Plan

HNF-SP-1242, Rev. 0

\section{HANFORD SITE WORK BREAKDOWN STRUCTURE DICTIONARY LANDLORD}

WBS: 1.5

\begin{tabular}{|l|l|l|c|}
\hline $\begin{array}{l}\text { 1. Activity Title: } \\
\begin{array}{l}\text { Core Infrastructure Maintenance - } \\
\text { Project L-288 }\end{array}\end{array}$ & $\begin{array}{l}\text { 2 Date } \\
\text { July } 1997\end{array}$ & $\begin{array}{c}\text { 4. Dict. Rev. } \\
\text { RL-TP13 }\end{array}$ \\
\hline 5. Contract WBS No. & $\begin{array}{l}\text { 6. Corresponding } \\
\text { FDS No. }\end{array}$ & 7. Baseline CR No. \\
1.5.1.1.2.9F & 9. B \& R No. & \\
\hline 8. Organization Name & EW7070201 \\
DynCorp Operations &
\end{tabular}

10. Scope of Work

This capital small project will provide the engineering, inspection, and construction for a replacement fire protection water line and sanitary sewer tie-in for the meteorological station facilities near the 200 West Area. The project includes approximately 2,000 linear feet of 12-inch pressurized sewer line to the 3,400 linear feet of 2-inch pressurized sewer line to the existing meteological station. The current septic system does not meet current Washington State environmental regulations (WAC 246-272, "Onsite Sewage Systems"). Also, the current fire protection line to these facilities comes directly off the 200 West export water line, which is not energy efficient and requires a higher pressure be maintained on the export water line. 


\section{LANDLORD PROJECT}

WBS 1.5

FY 1998 Work Plan

HNF-SP-1242, Rev. 0

\section{HANFORD SITE WORK BREAKDOWN STRUCTURE DICTIONARY LANDLORD}

WBS: 1.5

\begin{tabular}{|l|l|l|c|}
\hline $\begin{array}{l}\text { 1. Activity Title: } \\
\begin{array}{l}\text { Core Infrastructure Maintenance - } \\
\text { Project L-289 }\end{array}\end{array}$ & $\begin{array}{l}\text { 2 Date } \\
\text { July } 1997\end{array}$ & $\begin{array}{l}\text { 3. PBS Number } \\
\text { RL-TP13 }\end{array}$ & 0 \\
\hline 5. Contract WBS No. & $\begin{array}{l}\text { 6. Corresponding } \\
\text { FDS No. }\end{array}$ & 7. Baseline CR No. \\
1.5.1.1.2.9G & 9. B \& R No. \\
\hline $\begin{array}{l}\text { 8. Organization Name } \\
\text { DynCorp Operations }\end{array}$ & EW7070201 & \\
\hline 10. Scope of &
\end{tabular}

10. Scope of Work

This workscope fixes the water drainage problem on Baltimore Avenue in the 200 East Area that was caused by construction activites during the past two years. This capital small project will design and install a storm water drainage system to meet safety and environmental requirements. 


\section{LANDLORD PROJECT \\ WBS 1.5}

FY 1998 Work Plan

HNF-SP-1242, Rev. 0

\section{HANFORD SITE WORK BREAKDOWN STRUCTURE DICTIONARY LANDLORD \\ WBS: 1.5}

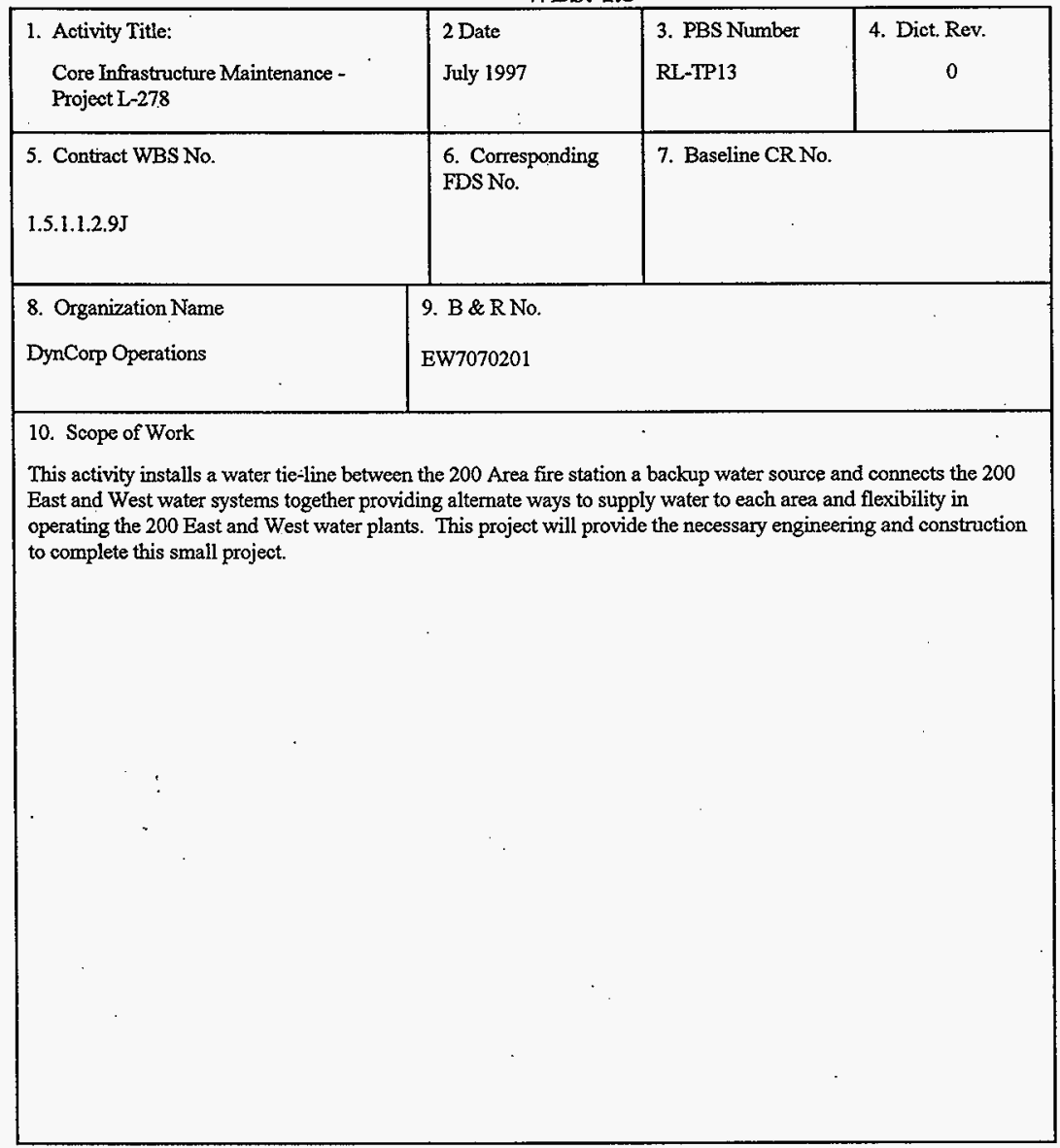

